\title{
Filtered Rings, Filtered Blowing-Ups and Normal Two-Dimensional Singularities with "Star-Shaped" Resolution
}

By

\author{
Masataka TOMARI $^{1}$ and Kei-ichi WATANABE ${ }^{2 *}$
}

\section{Table of Contents}

Introduction.

$\S 0 . \quad$ Notation and Conventions.

Chapter 1. Filtered Rings and Filtered Blowing-Ups.

$\S 1$. Filtered Blowing-Up and the Induced Filtration on Local Cohomology Groups.

$\S 2$. The Divisor Class Group of Normal Rees Algebras.

$\S 3$. The Dualizing Module on the Filtered Blowing-Up and the Criterion for Gorenstein Property.

Appendix to $\S 3$. Graded Dualizing Complex for Graded Rings and Duality for Projective Morphisms.

$\S 4$. Criterion for Normality and Rational Singularity

$\S 5$. The Case When $G$ Has an Isolated Singularity.

Chapter 2. Normal Two-Dimensional Singularities with "Star-Shaped" Resolution.

$\S 6 . ~ " S t a r-S h a p e d " ~ R e s o l u t i o n$ and the Filtration.

$\S 7$. Gorenstein Singularities with "Star-Shaped" Resolution.

$\S 8$. The Case with $p_{a}=2$.

References.

\section{Introduction}

The study of various blowing-ups of a local ring $(A, m)$ (or a "singularity" $(W, w)$, $W=\operatorname{Spec}(A)$ and $w=m)$ is very important in the theory of singularities. If we study the property of a singularity $(W, w)$ (e.g., resolution, invariants of $(W, w)$ defined by resolution, deformation, multiplicity, embedding dimension, ...), the study of the "tangent cone" (or the associated graded ring) $\bigoplus_{n \geq 0} m^{n} / m^{n+1}$ always plays an important role.

On the other hand, we have a fairly well-constructed theory of graded rings which enables us to know various ring theoretic properties of them (e.g., Cohen-Macaulay, Gorenstein property, rational singularity, divisor class group, ...) in terms of geometric language and which enables us to study the properties of normal graded rings in geometric terms.

So, our aim of this article is to provide a theory of filtration and filtered blowing-

Communicated by M. Kashiwara, February 4, 1987. Revised August 18, 1988.

1 Institute of Mathematics, University of Tsukuba, Tsukuba, Ibaraki 305, Japan.

2 Department of Mathematical Sciences, Tokai University, Hiratsuka, 259-12, Japan.

* Partially supported by Ishida Foundation. 
up for a general filtration on $(A, m)$ which enables us to know various ring theoretic properties and invariants of $(A, m)$ as a singularity. Our method is, roughly speaking, to approximate $(A, m)$ by a graded ring $G$ (given a filtration $\left\{F^{i}\right\}_{i \in \mathbb{Z}}$ on $A$ we have the associated graded ring $\left.G=\bigoplus_{n \in \mathbb{Z}} F^{n} / F^{n+1}\right)$. This explains the reason why we should consider more general filtration than an ideal-adic one. $A$ "good" filtration for us is one whose associated graded ring $G$ has good properties (normal, integral domain, reduced, ...), while in the case of $m$-adic filtration, $G$ does not have such properties in general.

As an excellent and an important example of our theory, we study normal twodimensional singularities with "star-shaped" resolutions. It turns out that if the associated graded ring of a filtration on a normal two-dimensional local ring $(A, m)$ is a graded domain which is finitely generated over the field $A / m$ with isolated singularity, then $A$ has a "star-shaped" resolution and, surprising enough, the filtration is also determined almost uniquely from this resolution (as in (5.13)). In this case, such filtration is not an ideal-adic one in general.

Now, we will summarize the contents of each section.

In Chapter 1, we develope general theory of a filtered blowing-up. Section 1 is devoted to the general definitions and fundamental properties of a filtered blowing-up and the induced filtration on the local cohomology groups. In Section 2, we study a filtration whose associated Rees algebra $\mathscr{R}$ is normal. We study the divisor class groups of the spaces which appear in our theory and also the relation between $\mathrm{Cl}(A)$ and $\mathrm{Cl}(\mathscr{R})$ (cf. Proposition (2.7)). This result is a generalization of that of [17]. In Section 3 , we calculate the dualizing module $\omega_{X}$ of the filtered blowing-up $X$ of $W$. In particular, if the associated graded ring $G$ is Gorenstein with $K_{G} \cong G(a)$, then we have $\omega_{X} \cong O_{X}(a+1)$ (cf. Theorem (3.5)). Also, the calculation of the canonical module of $\mathscr{R}$ shows that $K_{\mathscr{R}}$ is free if and only if $a=-2$, which gives another explanation of a result of Goto-Shimoda [11]. In Section 4, we give a lower bound of the geometric genus of a singularity in terms of the local cohomologies of $G$. Then we obtain numerical criteria for the filtered blowing-up $X$ of $W$ to be normal or has only rational singularities (Theorems (4.6) and (4.9)). In Section 5, we discuss the condition that $G$ has an isolated singularity. We prove: If $G$ has an isolated singularity, then the filtered blowing-up $X$ has only toric singularities which we call "cyclic quotient singularity" and, especially, for the case with $\operatorname{dim} A=2$ the dual graph of the exceptional locus of the minimal good resolution of $W$ is "star-shaped" (Theorem (5.6), see (0.5)).

In Chapter 2, we apply the results of Chapter 1 to the following situation:

Let $f:\left(\tilde{X}, f^{-1}(w)\right) \rightarrow(W, w)$ be a resolution of a normal two-dimensional singularity $(W, w)$ over a field with arbitrary characteristic such that the dual graph $\Gamma$ of $f^{-1}(w)$ is star-shaped and $\tau: \tilde{X} \rightarrow X$ be the contraction of $f^{-1}(w)$ except the central curve as in the followings:

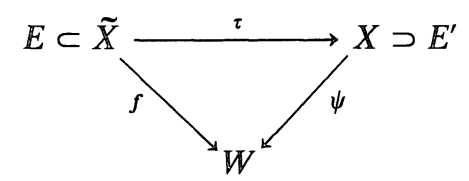


where $E$ is the central curve and $E^{\prime}$ is the image of $E$.

Let us introduce the filtration $\left\{F^{k}\right\}_{k \in \mathbf{Z}}$ on $A$, by $F^{k}=\psi_{*}\left(O_{X}\left(-k \cdot E^{\prime}\right)\right) \subset O_{W, w}=A$ for $k \in \mathbb{Z}$. Let $R(E, D)$ be the normal two-dimensional graded ring whose resolution graph $\Gamma$ is the same as that of $W$ (we call it Pinkham-Demazure's construction for $\Gamma$. See (6.2)). We consider the following problems:

Problem (1). Find good sufficient conditions for $(W, w)$ to be a small deformation of Pinkham-Demazure's construction.

Problem (2). Does the Gorenstein property of $A$ induce the Gorenstein property of $R(E, D)$ ? How is the converse? Can we find a criterion for $A$ to have the Gorenstein property only from the graph $\Gamma$ ?

Problem (3) (see Problems (7.6)). When $A$ is a Gorenstein domain, is $(W, w)$ a small deformation of $R(E, D)$ ?

In Section 6 , we see that $A$ and $R(E, D)$ are related by a filtration defined above in the following manner.

Theorem (6.3). Let the situation be as in (*). Then $G=\bigoplus_{k \geq 0}\left(F^{k} / F^{k+1}\right) T^{k}$ is an integral domain with isolated singularities. Further $R(E, D)$ is the normalization of $G$, and we have the exact sequence of graded $G$-modules

$$
0 \rightarrow G \rightarrow R(E, D) \rightarrow U \rightarrow 0
$$

with $U=\bigoplus_{k \geq 0} \operatorname{Ker}\left\{R^{1} \psi_{*}\left(O_{X}\left(-(k+1) E^{\prime}\right)\right) \rightarrow R^{1} \psi_{*}\left(O_{X}\left(-k \cdot E^{\prime}\right)\right)\right\} T^{k}$ (we can identify $E$ with $E^{\prime}$ by $\left.\tau\right)$ where $T$ denotes an indeterminate. Moreover $U$ has finite length and is isomorphic to $H_{G_{+}}^{1}(G)$, and $l_{A}(U)=p_{g}(R(E, D))-p_{g}(W, w)$.

In particular, $G$ is normal if and only if $p_{g}(R(E, D))=p_{g}(W, w)$.

For Problem (2), Theorem (7.2) characterizes the Gorenstein property of $(A, m)$ by the Gorenstein property of $R(E, D)$ and the injectivity of the canonical map $H^{1}\left(X, O_{X}(-a \cdot E)\right) \rightarrow H^{1}\left(X-E, O_{X}(-a \cdot E)\right) \cong H_{m}^{2}(A)$, where $a=a(R(E, D))$.

In the rest of this paper we study Problem (3). We have partial answers to this problem in Corollary (7.7), Theorems (7.8) and (7.9). In particular, the answer is yes in the case $p_{a}(\Gamma) \leq 1$ if the characteristic of the base field $k$ is zero (Corollary (7.10), see Notation and Conventions for $p_{a}(\Gamma)$ ). In Section 8, we examine Problem (3) for dual graphs with $p_{a}(\Gamma)=2$. Here we see that the answer is yes if the genus of central curve is greater than zero. Further we characterize the graph $\Gamma$ where Problem (3) still remains open in Lemma (8.11) (cf. Example (8.14)).

Both authors heartily thank to Prof. F. Hidaka for many discussions and to Prof. S. Goto for discussions about the canonical module of graded rings. The first named author heartily thanks to Prof. K. Saito for encouragements. 


\section{§0. Notation and Conventions}

$(0.1)$

$F=\left\{F^{k}\right\}_{k \in \mathbb{Z}}:$ a filtration on $(A, m)(1.1)$.

$\mathscr{R}=\bigoplus_{n \geq 0} F^{n} T^{n} \subset A[T]$ (1.1).

$\mathscr{R}^{\prime}=\bigoplus_{n \in \mathbb{Z}} F^{n} T^{n} \subset A\left[T, T^{-1}\right](1.1)$.

$N$ : the integer with $F^{n N}=\left(F^{N}\right)^{n}$ for $n \geq 0(1.1)$.

We assume $\operatorname{ht}\left(F^{N}\right) \geq 2$ in this paper and put $I=F^{N}(1.1)$.

$G=\bigoplus_{n \geq 0}\left(F^{n} / F^{n+1}\right) T^{n}=\mathscr{R}^{\prime} / u \mathscr{R}^{\prime}$, where $u=T^{-1}$ (1.1).

$W=\operatorname{Spec}(A)(1.2)$.

$X=\operatorname{Proj}(\mathscr{R})(1.2)$.

$E=\operatorname{Proj}(G)(1.2)$.

$Y=\operatorname{Spec}_{X}\left(\bigoplus_{n \geq 0} O_{X}(n)\right)(1.2)$

$Y^{\prime}=\operatorname{Spec}_{X}\left(\bigoplus_{n \in \mathbb{Z}} O_{X}(n)\right)(1.2)$.

$S$ is the "zero section" of $\pi: Y \rightarrow X$, i.e., the closed subscheme of $Y$ defined by $\left(\bigoplus_{n \geq 1} O_{X}(n)\right)^{\sim}$. We sometimes, by abuse of notations, omit $\sim$ as above; the ideal $\left(\bigoplus_{n \geq 1} O_{X}(n)\right)^{\sim}$ of
$O_{Y}$ is simply written as $\bigoplus_{n \geq 1} O_{X}(n)$.

$Z=\operatorname{Spec}(\mathscr{R})(1.2)$.

$Z^{\prime}=\operatorname{Spec}\left(\mathscr{R}^{\prime}\right)(1.2)$.

$a(A)=a_{F}(A)$ : a numerical invariant for a filtered $\operatorname{ring} A(1.12)$.

$K$ : the quotient field of the integral domain $A(2.1)$.

$J=P_{1}{ }^{\left(a_{1}\right)} \cap \cdots \cap P_{t}^{\left(a_{t}\right)}$ : the primary decomposition of $J$ in $\mathscr{R}$ with $G=\mathscr{R} / J=$ $\mathscr{R}^{\prime} / u \mathscr{R}^{\prime}(2.2)$.

$V_{i}$ : the valuation of $K(T)$ attached to $P_{i}(2.2)$.

$v_{i}$ : the normalized valuation of $K$ of the restriction of $V_{i}$ to $K$, with $V_{i}(x)=q_{i} v_{i}(x)$ for $x \in K$ and $q_{i} \in \mathbb{Z}$ (2.2).

$\mathrm{Cl}(U), \operatorname{cl}(D), F_{V}, E_{i}, F_{i}=F_{E_{i}},(2.6)$.

$K_{G}$ : the canonical module of $G[12]$.

$K_{\mathscr{R}}=H^{-d}\left(D_{\mathscr{R}}^{\circ}\right)$ : the canonical module of $\mathscr{R}$ (A.3.11).

$K_{\mathscr{R}^{\prime}}=H^{-d}\left(D_{\mathscr{R}^{\prime}}^{\circ}\right)$ : the canonical module of $\mathscr{R}^{\prime}$ (A.3.11).

$\omega_{X}$ : the dualizing module of $X$ (A.3.12) and (A.3.14).

$\omega_{Y}$ : the dualizing module of $Y(3.2)$.

$\omega_{E}$ : the dualizing module of $E[14]$. 
(0.4).

$p_{g}(A)=p_{g}(W, w)$ : the geometric genus of the singularity (4.1).

$U=\bigoplus_{k \geq 0} \operatorname{Ker}\left\{R^{1} \psi_{*}\left(O_{X}(k+1)\right) \rightarrow R^{1} \psi_{*}\left(O_{X}(k)\right)\right\}(5.6)$.

$R(E, D)$ : Let $D$ be a $\mathbf{Q}$-Cartier ample Weil divisor on a normal projective scheme $E$. Then we denote the normal domain $\bigoplus_{n \geq 0} H^{0}\left(E, O_{E}(n D)\right) T^{n}$ by $R(E, D)$ [7] (5.6). Any normal graded domain $R$ and $R_{0}$ being a field is written in this way [7]. Further in the case $\operatorname{dim}(R)=2$, Pinkham had written $D$ from the resolution graph $\Gamma$ [34] (6.2).

$$
Y^{\prime \prime}=\operatorname{Proj}\left(\left.\bigoplus_{l \geq 0} \mathscr{R}\right|_{l} \mathscr{R}^{\prime} U^{l}\right)(5.7) \text {. }
$$

For generalities concerning the dual graphs of exceptional sets for good resolutions of normal two-dimensional singularities, we refer $[43,25,26]$. Let $f:(\tilde{X}, A) \rightarrow(W, w)$ be a good resolution of normal two-dimensional singularity with exceptional locus $A$. Then

An irreducible component $E$ of $A$ is a central curve if genus $g(E) \geq 1$ or $E$ meets at least three other irreducible components of $A$.

The exceptional set $A$ (or the dual graph $\Gamma$ ) is called star-shaped if the dual graph is a tree with at most one central curve. For example,

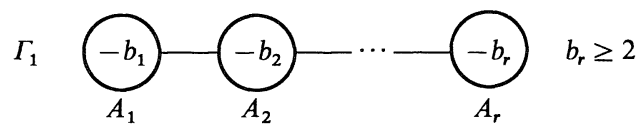

is star-shaped without central curves. (However, in this case we shall treat any component $A_{i}$ being as $E$ in Theorems of Chapter II.) Further

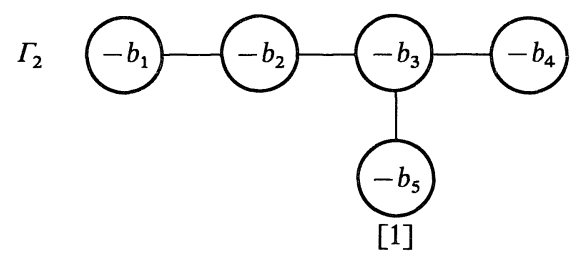

is not star-shaped, because there are two central curves in $\Gamma_{2}$.

By the star-shaped dual graph, we mean not only the topological weighted dual graph but also the graph with the analytic data so that two graphs $\Gamma$ and $\Gamma^{\prime}$ are same if and only if Pinkham-Demazure's constructions for both graphs are analytically isomorphic.

$L_{-k}$ : Giraud's inverse image of $-k E^{\prime}$ on $X$ by $\tau(6.11)$. 
$(0.7)$

$v:(C, E) \rightarrow\left(\operatorname{Spec}(R(E, D)), m_{R}\right):$ the canonical partial resolution of $\operatorname{Spec}(R(E, D))$ obtained as the filtered blowing-up (7.8).

$p_{a}(W, w)$ : the arithmetic genus of a singularity $(W, w)$ is the integer $p_{a}(W, w)$ defined via a resolution $f:(\tilde{X}, A) \rightarrow(W, w)$ by $p_{a}(W, w)=\sup \left\{p_{a}(D) \mid D\right.$ is a non-zero effective divisor on $\tilde{X}$ whose support is contained in $A\}$, where $p_{a}(D)$ is the virtual genus of $D$ [43]. This is determined by the graph $\Gamma$. Hence we shall denote it as $p_{a}(\Gamma)$.

In Chapter 2 (resp. Section 4), the ring $A$ is supposed to be essentially of finite type over an algebraically closed field $k$ (resp. over a field of characteristic zero).

For details about the materials above, we refer to the references cited after them. See $[25,26,28,41,43,49]$ for the basic facts on two-dimensional singularities and those numerical invariants.

\section{Chapter 1 . Fillered $\mathbb{R}$ ings and Filltered Blowing-Uns}

\section{§1. Filtered Blowing-Up and the Induced Filtration on Local Cohomology Groups}

(1.1) Throughout this chapter let $A$ be a Noetherian local ring with the maximal ideal $m$, which is a quotient of a Gorenstein ring (so that $A$ is universally catenary and has a dualizing complex). A filtration on $A$ is a decreasing sequence $\left\{F^{n}\right\}_{n \in \mathbb{Z}}$ of ideals of $A$ satisfying the following conditions:

(i) $F^{n} \neq(0)$ for every $n \in \mathbb{Z}, F^{n}=A$ for $n \leq 0$ and $\bigcap_{n \geq 0} F^{n}=(0)$.

(ii) $F^{i} \cdot F^{j} \subset F^{i+j}$ for every $i, j \in \mathbb{Z}$.

We define two "Rees algebras" for this filtration:

$\mathscr{R}=\bigoplus_{n \geq 0} F^{n} T^{n} \subset A[T]$ and

$\mathscr{R}^{\prime}=\bigoplus_{n \in \mathbb{Z}} F^{n} T^{n} \subset A\left[T, T^{-1}\right]$, where the symbol $T$ denotes an indeterminate.

We put $G=\bigoplus_{n \geq 0}\left(F^{n} / F^{n+1}\right) \cdot T^{n} \cong \mathscr{R}^{\prime} / u \cdot \mathscr{R}^{\prime}$, where $u$ denotes $T^{-1}$. We call $G$ the associated graded ring of $A$ with respect to $\left\{F^{n}\right\}_{n \in \mathbb{Z}}$.

In this paper we always assume that $\mathscr{R}$ is finitely generated over $A=\mathscr{R}_{0}$. This condition induces the relations $F^{n N}=\left(F^{N}\right)^{n}(n \geq 0)$ for some integer $N$ (Chapter 3 of [3]). Throughout this section, we will fix this integer $N$ and denote the ideal $\mathbb{F}^{N}$ by I. Further we will assume ht $(I) \geq 2$ in the below. Throughout this chapter we fix our filtration $\left\{F^{n}\right\}_{n \in \mathbb{Z}}$ and use the following notation.

(1.2) The fundamental diagram. We denote $W=\operatorname{Spec}(A), X=\operatorname{Proj}(\mathscr{R}), E=$ $\operatorname{Proj}(G), \quad Y=\operatorname{Spec}_{X}\left(\bigoplus_{n \geq 0} O_{X}(n)\right), Y^{\prime}=\operatorname{Spec}_{X}\left(\bigoplus_{n \in \mathbb{Z}} O_{X}(n)\right), Z=\operatorname{Spec}(\mathscr{R}), \mathbb{Z}^{\prime}=\operatorname{Spec}\left(\mathscr{R}^{\prime}\right)$, $S$ the closed subscheme of $Y$ defined by the ideal $\bigoplus_{n>0} O_{X}(n)$ of $O_{Y}$ (cf. (0.1)), where $\operatorname{Proj}(\mathscr{R})$ and $O_{X}(n)=(\mathscr{R}(n))^{\sim}$ are as in E.G.A. Chapter III. $\S 2,3,8$. Also we use $\mathbb{D}$ (resp. $D_{+}$) for open subsets of some "Spec" (resp. "Proj") and $V$ (resp. $V_{+}$) for closed subsets of some "Spec" (resp. "Proj"). 
Between these schemes, we have the canonical morphisms as indicated in the following commutative diagram:

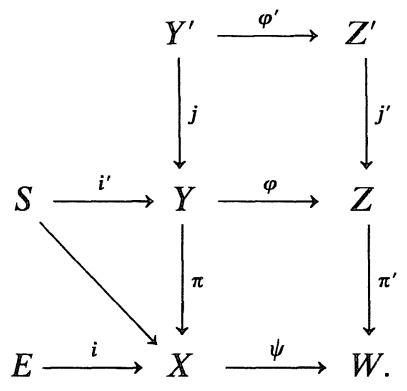

Here $X$ (resp. $Y, E$ ) is covered by affine open sets of the form $D_{+}\left(f^{*}\right)$ (resp. $\left.\pi^{-1}\left(D_{+}\left(f^{*}\right)\right), D_{+}(\bar{f})\right)$, where $f \in F^{d}-F^{d+1}$, with $d>0, \quad f^{*}=f T^{d} \in \mathscr{R}_{d}$ and $\bar{f}=$ $\left(f \bmod F^{d+1}\right) T^{d} \in G_{d}$ and $\Gamma\left(D_{+}\left(f^{*}\right), O_{X}\right)=F^{0}\left(A_{f}\right)\left(\right.$ resp. $\Gamma\left(\pi^{-1}\left(D_{+}\left(f^{*}\right)\right), O_{Y}\right)=\bigoplus_{n \geq 0} F^{n}\left(A_{f}\right) T^{n}$, $\left.\Gamma\left(D_{+}(\bar{f}), O_{E}\right)=F^{0}\left(A_{f}\right) / F^{1}\left(A_{f}\right)\right)$, where $F^{n}\left(A_{f}\right)=\bigcup_{s \geq 0}\left(F^{d s+n}\right) / f^{s}$ for every $n \in \mathbb{Z}$.

Note that our definition of $\left\{F^{n}\left(A_{f}\right)\right\}_{n \in \mathbf{Z}}$ gives a structure of filtered $A$-module on $A_{f}$ compatible with the filtration $\left\{F^{n}\right\}_{n \in \mathbb{Z}}$ on $A$.

Then the dual diagram of (1.2.1) over the affine open set $D_{+}\left(f^{*}\right)=D_{+}\left(f \cdot T^{d}\right)$ is as follows:

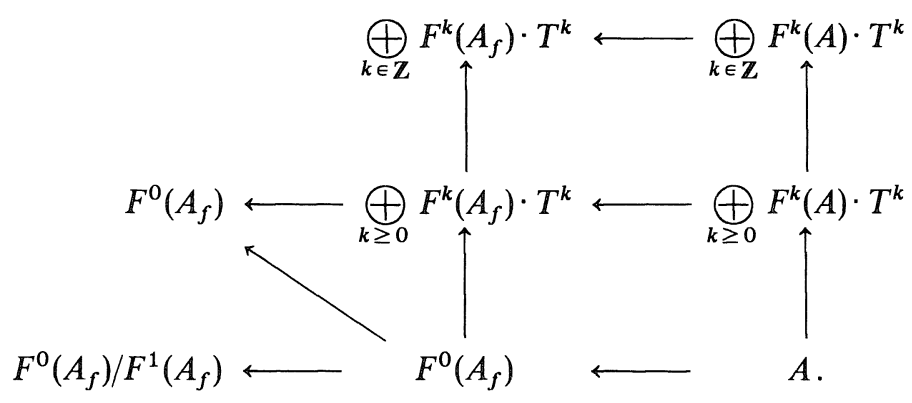

Concerning these morphisms, we have the following facts.

Remark (1.3). (i) The morphism $\psi$ is projective and induces an isomorphism between $X-E$ and $W-V(I)$.

(ii) The composition $\pi \cdot i^{\prime}$ is an isomorphism.

(iii) We can write the morphism $\varphi$ as follows: $Y \cong \operatorname{Proj}\left(\mathscr{R}^{\natural}\right) \stackrel{\varphi}{\longrightarrow} \operatorname{Spec}(\mathscr{R})$, where $\mathscr{R}^{\natural}=\left.\bigoplus_{m \geq 0} \mathscr{R}\right|_{m}$ with $\left.\mathscr{R}\right|_{m}=\bigoplus_{k \geq m} \mathscr{R}_{k}$. The exceptional locus of $\varphi$ is contained in $\varphi^{-1}\left(V\left(\left.\mathscr{R}\right|_{1}\right)\right)=\operatorname{Proj}\left(\bigoplus_{m \geq 0}\left(\left.\mathscr{R}\right|_{m}\right) /\left(\left.\mathscr{R}\right|_{m+1}\right)\right)=S$. Hence the morphism $\varphi$ induces an isomorphism between the schemes $Y-\left(S \cap \pi^{-1}(E)\right)$ and $Z-V\left(\mathscr{R}_{+}+I \cdot \mathscr{R}\right)$ by (i). Here we denote $\left.\mathscr{R}\right|_{1}$ also by $\mathscr{R}_{+}$.

(iv) The morphism $j$ is an open immersion with $j\left(Y^{\prime}\right)=Y-S$ and the composition $\varphi \cdot j$ gives an isomorphism of $Y^{\prime}$ and $Z-V\left(\mathscr{R}_{+}\right)$.

(v) Also the morphism $\varphi^{\prime}$ is an open immersion onto $Z^{\prime}-V\left(\mathscr{R}_{+} \cdot \mathscr{R}^{\prime}\right)$. 
(vi) ( 55 of [23]) There is an exact sequence of graded $\mathscr{R}$-modules

$$
0 \rightarrow H_{\mathscr{R}_{+}}^{0}(\mathscr{R}) \rightarrow \mathscr{R} \rightarrow \bigoplus_{n \in \mathbb{Z}} H^{0}\left(X, O_{X}(n)\right) \rightarrow H_{\mathscr{R}_{+}}^{1}(\mathscr{R}) \rightarrow 0
$$

and the isomorphisms $H_{\mathscr{R}+}^{q+1}(\mathscr{R}) \cong \bigoplus_{n \in \mathbb{Z}} \mathbb{H}^{q}\left(X, O_{X}(n)\right)$ for $q \geq 1$

(1.4) The following three conditions are equivalent; (i) $H_{\mathscr{R}_{+}}^{0}(\mathscr{R})=0$, (ii) $H_{I}^{0}(A)=0$, (iii) The canonical morphism $A \rightarrow H^{0}\left(X, O_{X}\right)$ is injective.

From now on, we assume these conditions.

Now we are interested in the condition so that we have the relation $H^{0}\left(Y, O_{Y}\right)=\mathscr{R}$ (resp. the relation $H^{0}\left(Y^{\prime}, O_{Y^{\prime}}\right)=\mathscr{R}^{\prime}$ ). For this purpose, we recall the following lemma.

Lemma (1.5). (i) If a Noetherian graded ring $\mathscr{R}$ satisfies the condition $\left(S_{k}\right)$ (resp. is normal, is a Cohen-Macaulay ring), so does (resp. is) $\operatorname{Proj}(\mathscr{R})$.

(ii) Let $X$ be a Noetherian scheme, $E$ a closed subscheme of $X$ with the open immersion $l: X-E \rightarrow X$. Then the relation $l_{*}\left(O_{X-E}\right)=O_{X}$ holds if and only if $\operatorname{depth}\left(O_{X, x}\right) \geq 2$ holds for every point $x$ of $E$.

Recall that we say that a coherent $O_{X}$-module $\mathscr{F}$ satisfies the Serre's condition $\left(S_{k}\right)$ if $\operatorname{depth}\left(\mathscr{F}_{X}\right) \geq \inf \left(k, \operatorname{dim}\left(\mathscr{F}_{X}\right)\right)$ for every point $x$ of $\mathbb{X}$.

Proof. (i) Take an element $f$ of $\mathscr{R}_{d}$ and examine the affine open set $D_{+}(f)$ of $\operatorname{Proj}(\mathscr{R})$. Then the coordinate ring of this affine open set is $\left(\mathscr{R}_{f}\right)_{0}=\left(\left(\mathscr{R}^{(d)}\right)_{f}\right)_{0}$, where $\mathscr{R}^{(d)}=\bigoplus_{k \geq 0} \mathscr{R}_{k d}$. Now, our conditions are inherited to $\mathscr{R}^{(d)}$ and also to the localizations. Since $\left(\mathscr{R}^{(d)}\right)_{f}$ is isomorphic to $\left(\left(\mathscr{R}_{f}\right)_{0}\right)\left[\mathbb{T}, T^{-1}\right]$, our conditions are inherited to $\left(\mathscr{R}_{f}\right)_{0}$.

(ii) See, E.G.A.IV, (5.10.5) [15].

Then we can show the following:

Proposition (1.6). Let the situation be as in (1.2). Then: (i) $\mathscr{R}^{\prime}$ is a CohenMacaulay (resp. Gorenstein) ring if and only if so is $G$.

(ii) $\mathscr{R}^{\prime}$ satisfies the condition $\left(S_{2}\right)$ if and only if $G$ satisfies the condition $\left(S_{1}\right)$ and $A$ satisfies the condition $\left(S_{2}\right)$.

(iii) $\mathscr{R}$ satisfies the condition $\left(S_{2}\right)$ if and only if so does $Y$ and the relation $\varphi_{*}\left(O_{Y}\right)=$ $O_{Z}\left(\right.$ or, $H^{0}\left(Y, O_{Y}\right)=\mathscr{R}$, i.e., $F^{k}=H^{0}\left(X, O_{X}(k)\right)$ for $\left.k \geq 0\right)$ holds.

(iv) If $\mathscr{R}^{\prime}$ satisfies the condition $\left(S_{2}\right)$, so does $\mathscr{R}$ (see Remark (1.8)). In this case $O_{X}$-modules $O_{X}(k)(k \in \mathbb{Z})$ satisfy the condition $\left(S_{2}\right)$.

Proof. (i) Let $u=\mathbb{T}^{-1}$ as usual. Then $u$ is a non-zero divisor of $\mathscr{R}^{\prime}$, and the relations $\mathscr{R}^{\prime} / u \cdot \mathscr{R}^{\prime} \cong G$ and $\left(\mathscr{R}^{\prime}\right)_{u} \cong A\left[T, T^{-1}\right]$ hold. Hence $\mathscr{R}^{\prime}$ is a Cohen-Macaulay (resp. Gorenstein) ring if and only if so are $G$ and $A$. Further it is well-known that $A$ is a Cohen-Macaulay (resp. Gorenstein) ring if $G$ is so (cf. [22], [6], [44]). Therefore our assertions follow.

(ii) follows by similar argument as in proof of (i).

(iii) If $\mathscr{R}$ satisfies the condition $\left(S_{2}\right)$, then so does $X$ by (i) of Lemma (1.5). Since $\mathscr{R}^{(d)}$ and $\mathscr{R}^{(d)}$-module $\mathscr{R}^{(d, n)}=\bigoplus_{n \geq 0} \mathscr{R}_{k+n d}$ for $k=0, \ldots, d-1$ are finite direct summands 
of $\mathscr{R}$, they also satisfy the condition $\left(S_{2}\right)$ as $\mathscr{R}^{(d)}$-modules. Let $f \in F^{d}-F^{d+1}$ and $f^{*}=f \cdot T^{d}$ as usual. Then $\left(\mathscr{R}^{(d, k)}\right)_{f^{*}}$ also satisfies the condition $\left(S_{2}\right)$ as $\left(\mathscr{R}^{(d)}\right)_{f^{*}}$ module. Here we have the relations $\left(\mathscr{R}^{(d, k)}\right)_{f^{*}}=F^{k}\left(A_{f}\right) \otimes_{F^{0}\left(A_{f}\right)}\left(\mathscr{R}^{(d)}\right)_{f^{*}}=$ $F^{k}\left(A_{f}\right) \otimes_{F^{0}\left(A_{f}\right)} F^{0}\left(A_{f}\right)\left[f^{*},\left(f^{*}\right)^{-1}\right]$. Hence $F^{k}\left(A_{f}\right)$ satisfies the condition $\left(S_{2}\right)$ as $F^{0}\left(A_{f}\right)$-module. Further $F^{k}\left(A_{f}\right) \otimes_{A} A\left[f^{*}\right]=\bigoplus_{n \geq 0} F^{k+n d}\left(A_{f}\right) \cdot T^{k+n d}$ satisfies the condition $\left(S_{2}\right)$ as $F^{0}\left(A_{f}\right) \otimes_{A} A\left[f^{*}\right]=\bigoplus_{n \geq 0} F^{n d}\left(A_{f}\right) \cdot T^{n d}$-module. Therefore $\bigoplus_{k \geq 0} F^{k}\left(A_{f}\right) \cdot T^{k}$ satisfies the condition $\left(S_{2}\right)$. This implies that $Y$ satisfies the condition $\left(S_{2}\right)$. Finally, by (iii) of Remark (1.3), (ii) of Lemma (1.5) and our assumption, we obtain the relation $\varphi_{*}\left(O_{Y}\right)=O_{Z^{\prime}}$, since both structure sheaves are the direct images of those on $Y-\left(S \cap \pi^{-1}(E)\right)$. Conversely, assume that $Y$ satisfies the condition $\left(S_{2}\right)$ and that the relation $H^{0}\left(Y, O_{Y}\right)=\mathscr{R}$ holds. Then $\mathscr{R}$ satisfies the condition $\left(S_{2}\right)$ by (iii) of Remark (1.3) and (ii) of Lemma (1.5).

(iv) First recall that $Y^{\prime}$ is isomorphic to an open subset of $Z^{\prime}$ by (v) of Remark (1.3). Hence $Y^{\prime}$ satisfies the condition $\left(S_{2}\right)$ if so does $\mathscr{R}^{\prime}$. Here we have the relation $\mathscr{R}^{\prime} / \mathscr{R}_{+} \mathscr{R}^{\prime} \cong\left(A / F^{1}\right)[u]$. Hence, by (v) of Remark (1.3) and (ii) of Lemma (1.5), we obtain $\mathscr{R}^{\prime}=H^{0}\left(Y^{\prime}, O_{Y^{\prime}}\right)$. This implies the relation $H^{0}\left(Y, O_{Y}\right)=\mathscr{R}$. Next $O_{Y^{\prime}}$ is a direct sum of $O_{Y^{\prime}}^{(d, k)}=\bigoplus_{n \in Z} O_{X}(n d+k) T^{n d+k}$ for $k=0, \ldots, d-1$ as $O_{Y^{\prime}}^{(d, 0)}$-module. Since $Y^{\prime}$ satisfies the condition $\left(S_{2}\right)$, the direct summands $O_{Y^{\prime}}^{(d, k)}$ with $k=0, \ldots, d-1$ also satisfy the condition $\left(S_{2}\right)$. Let $d$ be the integer such that $\mathscr{R}^{(d)}$ is generated by $\mathscr{R}_{d}$ over $A$. By using such $d$ in the situation above, we can conclude that $O_{X}(n)$ satisfies the condition $\left(S_{2}\right)$ for every integer $n$. Therefore $Y$ satisfies the condition $\left(S_{2}\right)$. Now, by (iii), $\mathscr{R}$ satisfies the condition $\left(S_{2}\right)$.

Proposition (1.7). Let the situation be as in (1.2).

(i) Assume that $Y$ satisfies the condition $\left(S_{2}\right)$ and that the quotient field of $G / P$ has a homogeneous element of degree 1 for every minimal prime $P$ of $G$. Then the canonical homomorphism

$$
O_{X}(m-n) \rightarrow \operatorname{Hom}_{O_{X}}\left(O_{X}(n), O_{X}(m)\right)
$$

is isomorphic for every integers $m$ and $n$.

(ii) Further suppose that $M$ is a finitely generated graded $\mathscr{R}$-module with $\operatorname{dim} M=$ $\operatorname{dim} \mathscr{R}$ satisfying the condition $\left(S_{2}\right)$, and put $F(n)=M(n)^{\sim}$ for every integer $n$. Then the canonical homomorphism

$$
F(m-n) \rightarrow \operatorname{Hom}_{O_{X}}\left(O_{X}(n), F(m)\right)
$$

is isomorphic for every integers $m$ and $n$.

Proof. (i) Our homomorphism $O_{X}(m-n) \rightarrow \operatorname{Hom}_{O_{X}}\left(O_{X}(n), O_{X}(m)\right)$ is induced by the multiplication. This is certainly isomorphic on $X-E$, because $O_{X}(n)=O_{X}$ on $X-E$ for every $n$. We have to show that this is isomorphic in the neighborhood of $E$. If $\mathscr{Q}$ is a graded prime ideal of height 1 of $\mathscr{R}$ corresponding to a generic point of $G$, then the homogeneous localization $\mathscr{R}_{(\mathscr{Q})}$ contains a unit of degree 1 by our assumption. This implies that $O_{X}(1)$ is invertible and $O_{X}(n) \cong O_{X}(1)^{\otimes n}$ for every $n$ at 2. So, our homomorphism is an isomorphism in codimension 1. Actually this is isomorphic, since both sides satisfy the condition $\left(S_{2}\right)$. 
Proof of (ii) goes all the same.

Remark (1.8). Let $A=k\left[\left[s^{4}, s^{3} t, s t^{3}, t^{4}\right]\right]$, where $k$ is a field and $s$ and $t$ are variables over $k$. If we put $F^{n}=\left(s^{4}, t^{4}\right)^{n}$ for every integer $n \geq 0$, then the associated Rees algebra $\mathscr{R}$ is Cohen-Macaulay [40]. However $\mathscr{R}^{\prime}$ does not satisfy the condition $\left(S_{2}\right)$ by our (ii) of Proposition (1.6) and the fact that $\operatorname{dim} A=2$ and $\operatorname{depth} A=1$. So the converse of (iv) of Proposition (1.6) is not true in general.

Remark (1.9). If $X$ satisfies the condition $\left(S_{2}\right), H^{0}\left(X, O_{X}\right)=A$ and if $\left\{F^{i}\right\}_{i \in \mathbb{Z}}$ is the $m$-adic filtration, then $A$ satisfies the condition $\left(S_{2}\right)$, too. In fact, take a non-zero divisor $x \in m$. If $y \in A$ and $y m \subset x A$, then $m \cdot(y / x) \subset m$ and $y / x \in H^{0}\left(X, O_{X}\right)=A$. Thus depth $A \geq 2$ and $A$ satisfies the condition $\left(S_{2}\right)$ at the other prime ideals since so does $X$.

It will be useful to know the condition for $X$ to be a Cohen-Macaulay scheme.

Proposition (1.10). Let the situation be as in (1.2). Then the following conditions are equivalent to each other.

(i) $Y$ is Cohen-Macaulay.

(ii) $Y^{\prime}$ is Cohen-Macaulay.

(iii) $G_{p}$ is Cohen-Macaulay for every homogeneous prime ideal $p$ of $G$ which does not contain $G_{+}$and $A_{q}$ is Cohen-Macaulay for every prime ideal $q$ of $A$ which does not contain I.

Proof. Let $f \in F^{N}-F^{N+1}$ and put $f^{*}=f \cdot T^{N}, \bar{f}=\left(f \bmod F^{N+1}\right) \cdot T^{N}$. Then $T^{-1}$ belongs to $\mathscr{R}_{f^{*}}=\mathscr{R}_{f^{*}}$. We have the relations $\mathscr{R}_{f^{*}} / T^{-1} \mathscr{R}_{f^{*}} \cong G_{\bar{f}}$ and $\left(\mathscr{R}_{f^{*}}\right)_{T^{-1}} \cong$ $\left(A_{f}\right)\left[T, T^{-1}\right]$. These isomorphisms prove the equivalence of (ii) and (iii).

The proof of the implication (i) from (ii) goes as in the proof of Proposition (1.6).

Remark (1.11). The equivalent conditions of Proposition (1.10) imply that $X$ is Cohen-Macaulay.

(1.12) Now, we shall discuss the filtration on the local cohomology groups induced from the filtration $\left\{F^{n}\right\}_{n \in \mathbb{Z}}$ as above. For an element $f$ of $F^{r}-F^{r+1}$ with integer $r \geq 1$, we will always denote $\bar{f}=\left(f \bmod F^{r+1}\right) T^{r} \in G_{r} T^{r}$ and $f^{*}=f T^{r} \in \mathscr{R}_{r}$ as in (1.2). Put $\operatorname{dim} G / m G=d$. Let $\bar{f}_{1}, \ldots, \bar{f}_{d}$ be a homogeneous system of parameters of $G / m G$ and $J$ the radical of $\left(\bar{f}_{1}, \ldots, \bar{f}_{d}\right)$ in $G$. Then $J+m G \supset G_{+}$holds. Hence $J \supset G_{+}$, and the radical of $\left(f_{1}^{*}, \ldots, f_{d}^{*}\right)$ (resp. $\left.\left(f_{1}, \ldots, f_{d}\right)\right)$ contains $\mathscr{R}_{+}$(resp. $\left.F^{1}\right)$. Therefore the scheme $E$ (resp. $X)$ is covered by affine open subsets $\left\{D_{+}\left(\overline{f_{i}}\right) \mid i=1, \ldots, d\right\}$ (resp. $\left\{D_{+}\left(f_{i}^{*}\right) \mid i=1\right.$, $\ldots, d\}$ ). Here we may (and we shall) assume that each $\bar{f}_{i}$ is a non-zero-divisor on $G / H_{G_{+}}^{0}(G)$ so that there exists an integer $n_{0}$ which satisfies the condition;

(1.12.1) If $\bar{x}$ belongs to $G_{n}$ with $n \geq n_{0}$ and $\bar{x} \neq 0$ (cf. Remark (1.14)), then $\bar{x} \cdot \overline{f_{i}} \neq 0$ for every $i$.

Now we define the Čech complex $C^{\bullet}$ (resp. $\bar{C}^{\circ}$ ) of $A$ (resp. of $G$ ) with respect to $\left(f_{1}, \ldots, f_{d}\right)$ (resp. $\left.\left(\bar{f}_{1}, \ldots, \bar{f}_{d}\right)\right)$ as follows: Let us denote the complex $\left[0 \rightarrow C_{i}^{0} \rightarrow C_{i}^{1} \rightarrow 0\right.$; where $C_{i}^{0}=A$ and $\left.C_{i}^{1}=A_{f_{l}}\right]$ (resp. $\left[0 \rightarrow \bar{C}_{i}^{0} \rightarrow \bar{C}_{i}^{1} \rightarrow 0\right.$; where $\bar{C}_{i}^{0}=G$ and $\left.\bar{C}_{i}^{1}=G_{f_{t}}\right]$ ) by $C_{i}^{*}\left(\right.$ resp. $\left.\bar{C}_{i}^{*}\right)$ for $i=1, \ldots, d$. Then we define the complex $C^{\circ}$ (resp. $\bar{C}^{\circ}$ ) by the tensor product of the complexes $C_{1}^{\circ}, \ldots, C_{d}^{\circ}$ over $A$ (resp. $\bar{C}_{1}^{\circ}, \ldots, \bar{C}_{d}^{\circ}$ over $G$ ).

Then, by taking the cohomology of these complexes, we have the following rela- 
tions (cf. Chapter III [15], [14]):

$$
H^{q}\left(C^{*}\right) \cong H_{I}^{q}(A) \quad \text { and } \quad H^{q}\left(\bar{C}^{*}\right) \cong H_{G_{+}}^{q}(G) \quad \text { for } \quad q \geq 0 .
$$

Here the $r$-th component $C^{r}$ of $C^{\circ}$ is a direct sum of localizations of $A$ for every $r>0$ and has the filtration on localizations defined naturally as in (1.2). These filtrations are preserved by boundary homomorphisms. So, $C^{\circ}$ is a filtered complex. Also, $\bar{C}^{\circ}$ is a complex of graded $G$-modules and we denote by $\left(\bar{C}^{\circ}\right)_{n}$ the complex induced from the homogeneous component of degree $n$ of $\bar{C}^{\circ}$.

Proposition (1.13). (i) The associated graded complex $G\left(C^{\circ}\right)$ of $C^{*}$ with respect to the filtration as above is isomorphic to $\bar{C}^{\circ}$. That is, $F^{n}\left(C^{\circ}\right) / F^{n+1}\left(C^{\circ}\right) \cong\left(\bar{C}^{\circ}\right)_{n}$ holds for every integer $n$.

(ii) For $n \in \mathbb{Z}$, we have the following exact sequence:

$$
0 \rightarrow H_{I}^{0}\left(F^{n}\right) \rightarrow F^{n} \rightarrow H^{0}\left(X, O_{X}(n)\right) \rightarrow H^{1}\left(F^{n}\left(C^{0}\right)\right) \rightarrow 0,
$$

and the isomorphisms:

$$
H^{q}\left(X, O_{X}(n)\right) \cong H^{q+1}\left(F^{n}\left(C^{*}\right)\right) \quad \text { for } \quad q \geq 1 .
$$

(iii) For $n \in \mathbb{Z}$, we have the isomorphisms:

$$
H^{q}\left(C^{\bullet} / F^{n}\left(C^{\bullet}\right)\right) \cong H_{E}^{q}\left(X, O_{X}(n)\right) \quad \text { for } \quad q \geq 0 .
$$

Proof. Our statement (i) follows almost directly from our definition of filtration.

If we define the complex $\left.C^{\circ}\right|_{1}$ replacing $C^{0}=A$ by 0 , we can easily show the equalities $H^{q+1}\left(F^{n}\left(\left.C^{\circ}\right|_{1}\right)\right)=H^{q}\left(X, O_{X}(n)\right)$ for $q \geq 0$ and $n \in \mathbb{Z}$.

Assertion (ii) follows from the exact sequence of complexes

$$
0 \rightarrow F^{n}\left(\left.C^{\circ}\right|_{1}\right) \rightarrow F^{n}\left(C^{\bullet}\right) \rightarrow F^{n} \rightarrow 0 .
$$

(iii) We have the exact sequence

$$
0 \rightarrow F^{n}\left(C^{\bullet}\right) \rightarrow C^{\bullet} \rightarrow C^{\bullet} / F^{n}\left(C^{\bullet}\right) \rightarrow 0 .
$$

Then the assertion follows from (i) and the long exact sequence of local cohomology groups.

Remark (1.14). Let $f$ be the product of a subset of $\left\{f_{1}, \ldots, f_{d}\right\}$ and $r$ the integer such that $f \in F^{r}-F^{r+1}$. Let $x$ be an element of $F^{n}-F^{n+1}$ with $n \geq n_{0}$ as in (1.12.1). Then $x / f^{s} \in F^{n-s r}-F^{n-s r+1}$ as $(\bar{f})^{t} \cdot \bar{x} \neq 0$ for every $t$ in $G$. Also, we can prove $\bigcap_{n \geq 0} F^{n}\left(A_{f}\right)=0$.

Here we introduce a numerical invariant $a(A)=a_{F}(A)$ for a filtered ring $A$ with filtration $F=\left\{F^{n}\right\}_{n \in \mathbb{Z}}$, which is analogous to the invariant $a(R)$ for a graded ring $R$ (cf. [12]).

Definition (1.15) (see also Proposition (1.17)). We define the filtration on local cohomology groups and the invariant $a(A)=a_{F}(A)$ as follows: 


$$
\begin{gathered}
F^{n}\left(H_{I}^{q}(A)\right)=\operatorname{Image}\left\{H^{q}\left(F^{n}\left(C^{\circ}\right)\right) \rightarrow H^{q}\left(C^{\circ}\right)\right\}, \\
a(A)=\max \left\{n \in \mathbb{Z} \mid F^{n}\left(H_{I}^{d}(A)\right) \neq 0\right\} .
\end{gathered}
$$

Remark (1.16). (i) This defines a decreasing filtration on $H_{I}^{q}(A)$ with

$$
F^{r} \cdot F^{n}\left(H_{I}^{q}(A)\right) \subset F^{n+r}\left(H_{I}^{q}(A)\right), \quad \text { for } n, r \in \mathbb{Z} \text {, and } q \geq 0 \text {. }
$$

(ii) $\lim H^{q}\left(F^{n}\left(C^{\circ}\right)\right)=H_{I}^{q}(A)$, where we take the limit with respect to decreasing order on $\overrightarrow{\mathbb{Z}}$ and the map induced from the inclusion of complexes $F^{m}\left(C^{\circ}\right) \rightarrow F^{n}\left(C^{\circ}\right)$ for $m>n$.

(iii) We have $H^{q}\left(F^{n}\left(C^{\circ}\right)\right)=0(q>1)$ for sufficiently large $n$, since $O_{X}(N)$ is a $\psi$-ample invertible sheaf (cf. (1.1) for the definition of $N$ ). Further we have $H^{1}\left(F^{n}\left(C^{\circ}\right)\right)=0$ for sufficiently large $n$ by Theorem (2.3.1), Chapter III of [15].

Although $\bar{C}^{\circ}$ is the associated graded complex of $C^{\circ}$, the operations of taking cohomology groups and associated graded modules are not commutative in general. So, we need some vanishing conditions to deduce the relations between $H_{I}^{q}(A)$ and $H_{G_{+}}^{q}(G)$.

Proposition (1.87)。 (i) If we identify $H_{I}^{q+1}(A)$ with $H^{q}\left(X-E, O_{X}\right)=H^{q}\left(X-E, O_{X}(n)\right)$ for $q \geq 1$, then $F^{n}\left(H_{I}^{q+1}(A)\right)$ is the image of $H^{q}\left(X, O_{X}(n)\right)$ in $H^{q}\left(X-E, O_{X}(n)\right)$. In particular, this filtration does not depend on the choice of homogeneous system of parameters $\left\{f_{1}, \ldots f_{d}\right\}$.

(ii) Suppose $H_{G_{+}}^{q-1}(G)=0$ and $H_{G_{+}}^{q+1}(G)=0$ for some integer $q \geq 1$. Then $H_{G_{+}}^{q}(G)$ is the associated graded module of $H_{I}^{q}(A)$. Namely, $F^{n}\left(H_{I}^{q}(A)\right) / F^{n+1}\left(H_{I}^{q}(A)\right) \cong\left[H_{G_{+}}^{q}(G)\right]_{n}$ holds for every $n \in \mathbb{Z}$. In particular, if $H_{G_{+}}^{d-1}(G)=0$, then the equality $a(A)=a(G)$ holds. (In general, we have the relation $a(A) \leq a(G)$ ).

(iii) If $H_{G_{+}}^{q}(G)=0$, then $H_{I}^{q}(A)=0$ and $H^{q}\left(F^{n}\left(C^{\circ}\right)\right)=0$ for every $n \in \mathbb{Z}$.

(iv) Let $q$ and $n$ be some integers with $q<d$. Suppose $\left[H_{G_{+}}^{q}(G)\right]_{i}=0$ for $i<n$. Then the canonical morphism $H^{q}\left(X, O_{X}(n)\right) \rightarrow H^{q}\left(X-E, O_{X}(n)\right)$ is injective.

Proof. The assertion (i) follows from Proposition (1.13). The assertion (ii) follows from the cohomology exact sequence associated to the exact sequence of complexes

$$
0 \rightarrow F^{n+1}\left(C^{\circ}\right) \rightarrow F^{n}\left(C^{\circ}\right) \rightarrow(\bar{C})_{n} \rightarrow 0 .
$$

(iii) and (iv) follows from the following lemma which will also be used in $\S 4$.

Lemma (1.18). Let $q$ and $n_{1}$ be integers with $q>0$.

(i) Suppose $\left[H_{G_{+}}^{q}(G)\right]_{n}=H^{q}\left(\bar{C}_{n}^{\circ}\right)=0$ for every $n \geq n_{1}$. Then $H^{q}\left(F^{n}\left(C^{\circ}\right)\right)=0$ for every $n \geq n_{1}$.

(ii) Suppose $\left[H_{G_{+}}^{q}(G)\right]_{n}=H^{q}\left(\bar{C}_{n}^{\circ}\right)=0$ for every $n<n_{1}$. Then $H^{q}\left(C^{\circ} / F^{n}\left(C^{\circ}\right)\right)=0$ and the canonical homomorphism $H^{q+1}\left(F^{n}\left(C^{*}\right)\right) \rightarrow H^{q+1}\left(C^{*}\right)$ is injective for every $n \leq n_{1}$.

Proof. (i) By the exact sequence (1.17.1) and our assumption, the inclusion $F^{m}\left(C^{\circ}\right) \rightarrow F^{n}\left(C^{\circ}\right)$ induces the surjective map $H^{q}\left(F^{m}\left(C^{\circ}\right)\right) \rightarrow H^{q}\left(F^{n}\left(C^{*}\right)\right)$ for every $m>n \geq$ $n_{1}$. By (iii) of $\operatorname{Remark}(1.16)$, this shows the relation $H^{q}\left(F^{n}\left(C^{\circ}\right)\right)=0$ for $n \geq n_{1}$. 
(ii) Consider the sequence of complexes

$$
0 \rightarrow \bar{C}_{n}^{\bullet} \rightarrow C^{*} / F^{n+1}\left(C^{*}\right) \rightarrow C^{*} / F^{n}\left(C^{*}\right) \rightarrow 0 .
$$

Our assumption implies that the canonical surjection $C^{*} / F^{m}\left(C^{*}\right) \rightarrow C^{*} / F^{n}\left(C^{*}\right)$ induces the injective map $H^{q}\left(C^{*} / F^{m}\left(C^{*}\right)\right) \rightarrow H^{q}\left(C^{*} / F^{n}\left(C^{*}\right)\right)$ for every $n<m \leq n_{1}$.

Since $\lim _{3} H^{q}\left(C^{*} / F^{n}\left(C^{*}\right)\right)=0$, where the limit is taken as in (1.16)(ii), the injectivity shows the relation $H^{q}\left(C^{*} / F^{n}\left(C^{*}\right)\right)=0$ for every $n \leq n_{1}$. The latter half of the statement follows directly from this vanishing.

\section{§2. The Divisor Class Group of Normal Rees Algebras}

(2.1) In this section, we always assume that the Rees algebra $\mathscr{R}$ is normal. Then our filtration can be described by certain family of valuations on the quotient field $K$ of $A$ (2.2). Our goal in this section is Proposition (2.7) which is a generalization of Theorem (1.6) of [45] and [17].

Note that $\mathscr{R}^{\prime}$ is normal when $\mathscr{R}$ is normal. Also note that $\mathscr{R}$ is normal when $A$ is normal and $G$ is reduced (cf. Proposition (1.6)).

(2.2) First, we define $J$ of $\mathscr{R}$ by: $J=\bigoplus_{n \geq 0} F^{n+1} T^{n}$, so that $\mathscr{R} / J \cong G \cong \mathscr{R}^{\prime} / u \mathscr{R}^{\prime}$. Since $\mathscr{R}^{\prime}$ is normal, the principal ideal $u \mathscr{R}^{\prime}$ is unmixed of height one. Hence $J$ is also unmixed of height one. Let

$$
J=P_{1}^{\left(a_{1}\right)} \cap \cdots \cap P_{t}^{\left(a_{t}\right)}
$$

be the primary decomposition of $J$ and $V_{i}$ the valuation of $K(T)$ attached to $P_{i}$, $1 \leq i \leq t$. By the relation $J=u \mathscr{R}^{\prime} \cap \mathscr{R}$, the integer $a_{i}$ is given by $a_{i}=V_{i}(u)$ for $1 \leq i \leq t$. If $v_{i}$ is the normalized valuation on $K$ of the restriction of $V_{i}$ on $K$, we have the relation $V_{i}(x)=q_{i} \cdot v_{i}(x)$ for every $x$ of $K$ with some positive integer $q_{i}$ for $1 \leq i \leq t$. Since we have the relations

$$
P_{1}^{\left(k \cdot a_{1}\right)} \cap \cdots \cap P_{t}^{\left(k \cdot a_{t}\right)}=u^{k} \mathscr{R}^{\prime} \cap \mathscr{R}=\bigoplus_{n \geq 0} F^{n+k} T^{n} \text { and } F^{k}=u^{k} \mathscr{R}^{\prime} \cap A \text { for } k \in \mathbb{Z},
$$

we can describe $F^{k}$ as follows:

$$
\begin{aligned}
F^{k} & =\left\{x \in A \mid V_{i}(x) \geq k \cdot a_{i}, 1 \leq i \leq t\right\} \\
& =\left\{x \in A \mid v_{i}(x) \geq k \cdot\left(a_{i} / q_{i}\right), 1 \leq i \leq t\right\} .
\end{aligned}
$$

Conversely, we obtain the following:

Lemma (2.3). Let $A$ be a Noetherian normal domain with the quotient field $K$ and $v_{1}, \ldots, v_{s}$ be discrete valuations on $K$ which satisfy the conditions: $v_{i}(x) \geq 0$ for $x \in A$, $1 \leq i \leq s$. Suppose that the positive rational numbers $r_{1}, \ldots, r_{s}$ are given and that the filtration $\left\{F^{k}\right\}_{k \in \mathbf{Z}}$ is defined by

$$
F^{k}=\left\{x \in A \mid v_{i}(x) \geq k \cdot r_{i}, 1 \leq i \leq s\right\} .
$$

Then the associated Rees algebra $\mathscr{R}=\bigoplus_{k \geq 0} F^{k} T^{k}$ is normal. (However, $\mathscr{R}$ is not necessarily Noetherian, in general, in this case.) 
Proof. Let us write $r_{i}=p_{i} / q_{i}$ with relatively prime positive integers $p_{i}$ and $q_{i}$, $1 \leq i \leq s$. Define the valuation $V_{i}$ on $K(T)$ by the rule; $V_{i}(x)=q_{i} v_{i}(x)$ for $x \in K$ and $V_{i}(T)=-p_{i}, 1 \leq i \leq s$. Then our $\mathscr{R}$ is written as follows:

$$
\mathscr{R}=\left\{f \in A[T] \mid V_{i}(f) \geq 0,1 \leq i \leq s\right\} .
$$

Hence $\mathscr{R}$ is a Krull domain.

The following remark gives the condition to get Noetherian $\mathscr{R}$ (cf. Chapter III [15]):

Remark (2.4). (i) Let $f: W^{\prime} \rightarrow W=\operatorname{Spec}(A)$ be a projective birational morphism with normal $W^{\prime}$ and normal $W$. Let $D$ be an effective rational coefficient Weil divisor on $W^{\prime}$ such that there is a positive integer $N$ so that $-N \cdot D$ is an $f$-ample Cartier divisor on $W^{\prime}$. If we define the filtration $\left\{F^{n}\right\}_{n \in \mathbb{Z}}$ by:

$$
F^{k}=\left\{x \in A \mid \operatorname{div}_{W^{\prime}}\left(f^{*}(x)\right) \geq k \cdot D\right\},
$$

then the attached Rees algebra $\mathscr{R}$ is a Noetherian normal graded domain and $\operatorname{Proj}(\mathscr{R})=W^{\prime}$.

(ii) Conversely, every our filtrations of (2.2) can be obtained in this way. That is: Put $W^{\prime}=\operatorname{Proj}(\mathscr{R})$ and $D=\sum_{i=1}^{t}\left(a_{i} / q_{i}\right) E_{i}$, where $E_{i}$ is the irreducible closed subvariety of $W^{\prime}$ defined by the homogeneous prime ideal $P_{i}, 1 \leq i \leq t$.

Example (2.5). (a) Let $A=k[[x, y, z]] /\left(x^{2}+y^{3}+z^{3}\right)$, where $k$ is a field and $m$ be the maximal ideal of $A$. Then the Rees algebra of $A$ with respect to the $m$-adic filtration is normal, since $m^{k}$ is integrally closed for every $k$ as $A$ is a rational singularity ((7.1) of $[28])$.

On the other hand, if we define another filtration on $A$ by: $F^{k}(A)=m^{\{k / 2\}}$, where $\{k / 2\}$ is the smallest integer not less than $k / 2$, then the corresponding Rees algebra is not normal. In fact, $x^{2}=-y^{3}-z^{3} \in m^{3}=F^{6}$, while $x \notin F^{3}=m^{2}$.

Furthermore, if we define the new filtration $\left\{\bar{F}^{k}\right\}_{k \in \mathbb{Z}}$ so that $\bigoplus_{k \geq 0} \bar{F}^{k} T^{k}$ is the normalization of $\bigoplus_{k \geq 0} F^{k} T^{k}$ in $K[T]$, then this filtration coincides with the one induced by grading of $A$ defined by: $\operatorname{deg}(x)=3$ and $\operatorname{deg}(y)=\operatorname{deg}(z)=2$.

In this example, "Proj" of the three Rees algebras are the same scheme.

(b) Let $A=k[[x, y, z]] /\left(x^{2}+y^{3}+z^{4}\right)$ and define the filtrations $\left\{F^{k}\right\}_{k \in \mathbb{Z}}$ and $\left\{\bar{F}^{k}\right\}_{k \in \mathbb{Z}}$ by the same process as above. In this example, the associated graded ring of $\left\{\bar{F}^{k}\right\}_{k \in \mathbb{Z}}$ is $k[x, y, z] /\left(x^{2}+y^{3}\right)$ with: $\operatorname{deg}(x)=3$ and $\operatorname{deg}(y)=2 \operatorname{deg}(z)=2$ (cf. Example $(6.19))$.

(2.6) Now, we will investigate the divisor class groups of $\mathscr{R}$ and $\mathscr{R}^{\prime}$ using the diagram (1.2.1). We will denote by:

$\operatorname{Div}(U)$ (resp. $P(U)$ ): the group of divisors (resp. principal divisors) of a normal scheme $U$

$\mathrm{Cl}(U)$ : the divisor class group of a normal scheme $U$

$\operatorname{cl}(D)$ : the class of a Weil divisor $D$ of $U$ in $\mathrm{Cl}(U)$.

If $V$ is a prime divisor of $X$, we put: 
$F_{V}$ : the prime divisor $\left(\pi^{-1}(V)\right)_{\text {red }}$ of $Y$.

For $P_{i}(1 \leq i \leq t)$ of $(2.2)$, we denote:

$E_{i}$ : the prime divisor of $X=\operatorname{Proj}(\mathscr{R})$ defined by $P_{i}$

$F_{i}:=F_{E_{i}}, 1 \leq i \leq t$.

The morphism $\pi$ induces the homomorphism $\pi^{*}: \operatorname{Div}(X) \rightarrow \operatorname{Div}(Y)$ so that $\pi^{*}\left(E_{i}\right)=$ $q_{i} \cdot F_{i}, 1 \leq i \leq t$, and $\pi^{*}(V)=F_{V}$ for $V \neq E_{i}(1 \leq i \leq t)$.

Proposition (2.7). (i) We have the isomorphisms:

$$
\begin{gathered}
\mathrm{Cl}(\mathscr{R}) \cong \mathrm{Cl}(Y), \quad \text { and } \\
\mathrm{Cl}\left(\mathscr{R}^{\prime}\right) \cong \mathrm{Cl}\left(Y^{\prime}\right) \cong \mathrm{Cl}(Y) / \mathbb{Z} \operatorname{cl}(S)
\end{gathered}
$$

(ii) We have the exact sequences:

$$
0 \rightarrow \bigoplus_{i=1}^{t} \mathbf{Z} \cdot E_{i} \rightarrow \mathrm{Cl}(Y) \rightarrow \mathrm{Cl}(A) \rightarrow 0
$$

and

$$
0 \rightarrow \mathrm{Cl}(X) \rightarrow \mathrm{Cl}(Y) \rightarrow \bigoplus_{i=1}^{t} \mathbb{Z} / q_{i} \mathbb{Z} \rightarrow 0
$$

(iii) There are exact sequences:

$$
0 \rightarrow \mathbb{Z}^{t} \rightarrow \mathrm{Cl}(\mathscr{R}) \rightarrow \mathrm{Cl}(A) \rightarrow 0
$$

and

$$
0 \rightarrow \operatorname{Coker}(\alpha) \rightarrow \mathrm{Cl}\left(\mathscr{R}^{\prime}\right) \rightarrow \mathrm{Cl}(A) \rightarrow 0,
$$

where $\alpha: \mathbf{Z} \rightarrow \mathbb{Z}^{t}$ is defined by $\alpha(1)=\left(a_{1}, \ldots, a_{t}\right)$. In particular, $\mathscr{R}^{\prime}$ is factorial if and only if $A$ is factorial, $t=1$ and $a_{1}=1$ (or, equivalently, if and only if $A$ is factorial and $G$ is an integral domain).

Proof. (i) By (1.3), (iv) and (v), $\varphi$ and $\varphi^{\prime}$ are isomorphisms in codimension one. Hence they induce isomorphisms of divisor class groups.

(ii) Let $\operatorname{HDiv}(Y)$ be the subgroup of $\operatorname{Div}(Y)$ generated by "homogeneous" divisors of $Y$ : That is, the subgroup of $\operatorname{Div}(Y)$ generated by $S$ and the set $\left\{F_{V} \mid V\right.$ is a prime divisor of $X\}$. Also let $\operatorname{HP}(Y)$ be the subgroup of $\operatorname{Div}(Y)$ generated by the set $\left\{\operatorname{div}_{Y}(f) \mid f\right.$ is a homogeneous element of $\left.K\left[T, T^{-1}\right]\right\}$. Then, by (7.1) of [39], we have the relation $\mathrm{Cl}(Y) \cong \operatorname{HDiv}(Y) / \mathrm{HP}(Y)$. From the relations $\mathrm{HP}(Y) \cong P(X) \oplus \mathbf{Z} \operatorname{div}_{Y}(T)$ and $\operatorname{div}_{Y}(T)=-\operatorname{div}_{Y}(u)=S-\sum_{i=1}^{t} a_{i} F_{i}$, we can easily see that $\mathrm{Cl}(Y)$ is generated by the set $\left\{\operatorname{cl}\left(F_{V}\right) \mid V\right.$ is a prime divisor of $\left.X\right\}$. Our assertion follows from the relations $\pi^{*}\left(\operatorname{cl}\left(E_{i}\right)\right)=q_{i} \cdot \operatorname{cl}\left(F_{i}\right), 1 \leq i \leq t$, and $\pi^{*}(\operatorname{cl}(V))=\operatorname{cl}\left(F_{V}\right)$ for prime divisor $V$ of $X$ with $V \neq E_{i}, 1 \leq i \leq t$.

(iii) Since $\pi$ has no ramification outside $E$, we have $H \cdot \operatorname{Div}(Y) \cong \pi^{*} \psi^{*}(\operatorname{Div}(A)) \oplus$ $\left(\sum_{i=1}^{t} \mathbf{Z} \cdot E_{i}\right) \oplus Z \cdot S \quad\left(\right.$ resp. $\left.\quad H \cdot \operatorname{Div}\left(Y^{\prime}\right) \cong \pi^{*} \psi^{*}(\operatorname{Div}(A)) \oplus\left(\sum_{i=1}^{t} \mathbf{Z} \cdot E_{i}\right)\right)$. The exact sequences follow in the same manner as in (ii).

Q.E.D. 
Example (2.8). Let $A=k[X, Y]^{(n)}$ and $\left\{F^{k}\right\}_{k \in \mathbb{Z}}$ be the $m$-adic filtration on $A$, where $m=(X, Y)^{(n)}$. Then $\mathrm{Cl}(\mathscr{R}) \cong Z$ and $\mathrm{Cl}(A) \cong \mathbb{Z} / n \mathbb{Z}$. This shows that the first exact sequence in (2.7) (iii) does not split in general.

Remark (2.9). The characterization of the filtrations satisfying our conditions can be found in [36]. The calculation of $\mathrm{Cl}(\mathscr{R})$ and $\mathrm{Cl}\left(\mathscr{R}^{\prime}\right)$ in the case $t=1$ and $a_{1}=1$ can be found in [17] also in [5], [18] under certain conditions. Also S. Itoh orally showed us a simple ring-theoretic computation method for $\mathrm{Cl}(\mathscr{R})$ and $\mathrm{Cl}\left(\mathscr{R}^{\prime}\right)$ under the same assumption as ours.

\section{§3. The Dualizing Module on the Filtered Blowing-Up and the Criterion for Gorenstein Property}

(3.1) In this section, we keep the notation of $\S 1$ and let $D_{X}^{\circ}$ (resp. $\omega_{X}$ ) be the dualizing complex (resp. the dualizing module) of $X=\operatorname{Proj}(\mathscr{R})$ (see Appendix to this section, for the generalities of such materials). The aim of this section is to express the module $\omega_{X}$ in terms of known data and to relate to some criterion for $G$ or $\mathscr{R}$ to be Gorenstein.

Proposition (3.2)。 The dualizing module $\omega_{Y}$ of $Y$ is given by $\omega_{Y} \cong \bigoplus_{n>0} \omega_{X}(n)$.

Proof. Let $Y^{(N)}=\operatorname{Spec}_{X}\left(\bigoplus_{n \geq 0} O_{X}(n N)\right)$. Since $O_{X}(N)$ is invertible, the dualizing sheaf on $Y^{(N)}$ is given by $\bigoplus_{n>0} \omega_{X}(n N)$ (p. 144 of [16]). Since the canonical morphism $Y \rightarrow Y^{(N)}$ is finite, $\omega_{Y}$ is given by $\omega_{Y}=\operatorname{Hom}_{O_{\left(Y^{(N)}\right)}}\left(O_{Y}, \omega_{\left(Y^{(N)}\right)}\right)$. This formula gives us our desired result. (cf. Proof of Proposition (1.6) of [46]).

Corollary (3.3).

(i) $\omega_{Y^{\prime}} \cong \bigoplus_{n \in \mathbb{Z}} \omega_{X}(n)$.

(ii) $K_{\mathscr{R}} \cong \bigoplus_{n>0} H^{0}\left(X, \omega_{X}(n)\right)$.

(iii) $K_{\mathscr{R}^{\prime}} \cong \bigoplus_{n \in \mathbb{Z}} H^{0}\left(X, \omega_{X}(n)\right)$.

Proof. (i) is given by localizing (3.2).

(ii) and (iii) $\varphi$ and $\varphi^{\prime}$ are both isomorphisms in codimension one. Since the canonical module always satisfy the condition $\left(S_{2}\right)$, we obtain the relations $\varphi_{*}\left(\omega_{Y}\right)=\omega_{Z}$ and $\varphi^{\prime}{ }_{*}\left(\omega_{Y^{\prime}}\right)=\omega_{Z^{\prime}}$. Hence the assertions follow.

Proposition (3.4). Assume that $G$ is Cohen-Macaulay. Then $K_{G} \cong\left(K_{\mathscr{R}^{\prime}} / u K_{\mathscr{R}^{\prime}}\right)(-1)$.

Proof. As $G$ is Cohen-Macaulay, so is $\mathscr{R}^{\prime}$ (cf. (1.6)). The normalized dualizing complex $D_{G}^{\circ}$ of $G$ is given by $D_{G}^{\circ}=\underline{\operatorname{RHom}}_{\mathscr{R}}\left(G, D_{\mathscr{R}^{\prime}}^{\circ}\right)$. From the exact sequence

$$
0 \rightarrow \mathscr{R}^{\prime}(1) \stackrel{u}{\rightarrow} \mathscr{R}^{\prime} \rightarrow G \rightarrow 0,
$$

we have the exact sequence of complexes

$$
0 \rightarrow D_{\mathscr{R}^{\prime}}^{\circ} \stackrel{u}{\rightarrow} D_{\mathscr{R}^{\prime}}^{\circ}(-1) \rightarrow D_{G}^{\cdot} \rightarrow 0
$$


Taking the cohomology groups, we have the desired result. Here note the vanishing $H^{-d}\left(D_{\mathscr{R}}^{\circ}\right)=0(d=\operatorname{dim} G)$ because $\mathscr{R}^{\prime}$ is Cohen-Macaulay.

Theorem (3.5). Suppose $G$ is Gorenstein with $a(G)=a$. Then:

(i) The relation $\omega_{X} \cong O_{X}(a+1)$ holds.

(ii) $K_{\mathscr{R}}$ is a free $\mathscr{R}$-module if and only if $a=-2$.

Proof. If $G$ is Gorenstein, we have the relation $G(a)=K_{G}$ by [12]. Then $\mathscr{R}^{\prime}$ is Gorenstein by (i) of Proposition (1.6), and $\mathbb{K}_{\mathscr{R}^{\prime}} \cong \mathscr{R}^{\prime}(a+1)$ by Proposition (3.4). So, by Corollary (3.3), $K_{\mathscr{R}}$ is isomorphic to $\bigoplus_{n>0} \mathscr{R}_{n+a+1}^{\prime}$. Thus we obtain the relations $\omega_{X}=\left(K_{\mathscr{R}}\right)^{\sim} \cong O_{X}(a+1)$. Since $\bigoplus_{n>0} \mathscr{R}_{n+a+1}^{\prime}$ is isomorphic to $\mathscr{R}$ if and only if $a=-2$, we obtain (ii).

Remark (3.6). (i) of Theorem (3.5) is a generalization of Theorem (5.4) of [41].

Proposition (3.7). Suppose $G$ is Cohen-Macaulay. Then, $\psi_{*}\left(\omega_{X}\right)$ is reflexive if and only if $a(G)<0$.

Proof. For every integer $n, \omega_{X}(n)$ and $\omega_{W}$ are isomorphic over $X-E$. Further $\varphi_{*}\left(\omega_{X}(n)\right)$ is reflexive $O_{W}$-module if $n$ is sufficiently small. Hence we obtain the relation $\varphi_{*}\left(\omega_{X}(n)\right)=\omega_{W}$ for sufficiently small $n$. By (ii) of Corollary (3.3) and Proposition (3.4), the relation $\varphi_{*}\left(\omega_{X}\right)=\omega_{W}$ holds if and only if $\left(K_{G}\right)_{n}=0$ for $n \leq 0$.

Corollary (3.8). Assume that $A$ is a rational singularity and that $G$ is CohenMacaulay. Then $a(A)=a(G)<0$ (cf. $\S 1$. for the definition of $a(A)$ and $\S 4$. for rational singularity.)

Proposition (3.9)*. Suppose that $G$ is Cohen-Macaulay and that $K_{G}$ is generated by the elements of degree not greater than one. Then $\omega_{X}$ is generated by $H^{0}\left(X, \omega_{X}\right)$ as an $O_{X}$-module.

Proof. By Proposition (3.4), we have the relation $K_{G} \cong\left(\left(K_{\mathscr{R}^{\prime}}\right) / u K_{\mathscr{R}^{\prime}}\right)(-1)$. Our assumption implies that $K_{\mathscr{R}^{\prime}}$ is generated by the elements of degree not greater than 0 over $\mathscr{R}^{\prime}$. So, there exists a free $\mathscr{R}$-module $L=\mathscr{R}\left(b_{1}\right) \oplus \cdots \oplus \mathscr{R}\left(b_{m}\right)$, where $b_{i} \geq 0$ for every $i$ and $a$ homomorphism $h: L \rightarrow K_{\mathscr{R}}$ for which $h_{n}: L_{n} \rightarrow\left(K_{\mathscr{R}}\right)_{n}$ is surjective for every $n \geq 0$. We can take a positive integer $N$ such that $\mathscr{R}_{b} \cdot \mathscr{R}_{N}=\mathscr{R}_{b+N}$ for $b \geq 0$. Hence $\left(K_{\mathscr{R}}\right)_{0}$ generates $\left(K_{\mathscr{R}}\right)^{(N)}$ as an $\mathscr{R}^{(N)}$-module. Therefore $\omega_{X}$ is generated by its global sections, because $H^{0}\left(X, \omega_{X}\right)=\left(K_{\mathscr{R}}\right)_{0}$ and $\omega_{X}=\left(K_{\mathscr{R}}\right)^{\sim}$.

(3.10) In the rest of this section we assume that $\mathscr{R}$ is normal, and describe the class of the dualizing sheaf as an element of the divisor class group. We preserve the notation in $\S 2$ in the below.

Proposition (3.11). We have the relation

$$
\operatorname{cl}\left(\omega_{Y}\right)=\pi^{*}\left(\operatorname{cl}\left(\omega_{X}\right)\right)+\operatorname{cl}\left(\sum_{i=1}^{t}\left(q_{i}-1\right) F_{i}-S\right) \quad \text { in } \mathrm{Cl}(Y) .
$$

\footnotetext{
* See Note Added in Proof (1).
} 
Proof. See Proposition (1.6) of [46].

Theorem (3.12). Suppose that $A$ is Gorenstein and that $G$ is an integral domain.

(i) Then there exists an integer a such that the relations $\omega_{X} \cong O_{X}(a+1)$ and $\omega_{E} \cong O_{E}(a)$ hold. In particular, if we put $\bar{G}=\bigoplus_{n \geq 0} H^{0}\left(E, O_{E}(n)\right)$, we have the relation $K_{\bar{G}} \cong \bar{G}(a)$.

(ii) Further suppose that $\bar{G}$ is Cohen-Macaulay. Then $\bar{G}$ is Gorenstein.

Proof. (i) Since the restriction $\left.\omega_{X}\right|_{X-E}$ is trivial and $E$ is irreducible, there is an integer $m$ such that the relations $\omega_{X} \cong O_{X}(-m E) \cong O_{X}(m)$ hold. By the adjunction formula, we have the relation $\omega_{E} \cong O_{E}(m-1)$.

(ii) See [12].

Corollary (3.13). (i) $K_{\mathscr{R}}$ is a free $\mathscr{R}$-module if and only if $\sum_{i=1}^{t}\left(1+a_{i}-q_{i}\right) / q_{i} E_{i}$ is an integral Weil divisor on $X$ and linearly equivalent to $K_{X}$ on $X$.

(ii) $K_{\mathscr{R}^{\prime}}$ is a free $\mathscr{R}^{\prime}$-module if and only if $\pi^{*}\left(K_{X}\right)+\sum_{i=1}^{t}\left(q_{i}-1\right) F_{i}$ is linearly equivalent to the integral divisor $m\left(\sum_{i=1}^{t} a_{i} F_{i}\right)$ for some integer $m$.

Proof. (i) $\mathrm{As} \mathrm{Cl}(\mathscr{R}) \cong \mathrm{Cl}(Y)$ and $\pi^{*}: \mathrm{Cl}(X) \rightarrow \mathrm{Cl}(Y)$ is injective, our result follows from Proposition (3.11) and the equality: $\operatorname{div}_{Y}(T)=S-\sum_{i=1}^{t} a_{i} F_{i}$.

(ii) follows from Proposition (3.11) and the isomorphism: $\mathrm{Cl}\left(Y^{\prime}\right) \cong \mathrm{Cl}(Y) /(\mathbb{Z} \mathrm{cl}(S))$.

Example (3.14). Put $\bar{G}=\bigoplus_{n \geq 0}\left(m^{n} / m^{n+1}\right)$ and assume that $\bar{G}$ is a Gorenstein domain with $a(\bar{G})=a$. If we define the filtration $\left\{F^{k}\right\}_{k \in \mathbb{Z}}$ by: $F^{k}=m^{\{k p / q\}}$ for $k \geq 0$, we have the relation $K_{X}=-(a+1) E$, because $X=\operatorname{Proj}(\mathscr{R})$ coincides with the $m$-adic blowing-up and $K_{Y}$ is linearly equivalent to the divisor $\{-q(a+1)+(q-1)-p\} F$, where $F=\pi^{-1}(E)_{\text {red }}$. Hence, $K_{\mathscr{R}}$ is free if and only if $p=-a q-1$.

For example, if $A=k[[x, y, z]] /\left(x^{2}-y z\right)$, we have $a(\bar{G})=-1$. Hence $K_{\mathscr{R}}$ is free if and only if $p=q-1$. In the case $q=2, \mathscr{R}=A\left[x T, y T, z T, x T^{2}, y T^{2}, z T^{2}\right]$, which turns out to be a Gorenstein ring.

Corollary (3.15). Assume that $I$ (see $\S 1)=F^{N}$ is $m$-primary and that $\mathscr{R}$ is normal Gorenstein. Further assume that $q_{i}=1$ holds for all $i$. Then $K_{A}$ is free and $K_{\mathscr{R}^{\prime}} / u K_{\mathscr{R}^{\prime}} \cong G(-1)$. Moreover, if $G$ is Cohen-Macaulay, then $G$ is Gorenstein with $a(G)=-2$.

Proof. By Corollary (3.13), $K_{X}$ is supported on $E$, hence $K_{A}$ is free. By the freeness of $K_{\mathscr{R}}, K_{X}$ is linearly equivalent to $\sum_{i=1}^{t} a_{i} E_{i}$ on $X$. In another word, $\omega_{X}$ is isomorphic to $O_{X}(-1)$. By (iii) of Proposition (1.6) and (iii) of Corollary (3.3), $K_{\mathscr{R}^{\prime}} \cong$ $\mathscr{R}^{\prime}(-1)$. Hence $G \cong \mathscr{R}^{\prime} / u \mathscr{R}^{\prime} \cong\left(K_{\mathscr{R}^{\prime}} / u K_{\mathscr{R}^{\prime}}\right)(1)$. The remaining assertion follows from Proposition (3.4). 


\section{Appendix to $§ 3$. Graded Dualizing Complex for Graded Rings and Duality for Projective Morphisms}

(A.3.1) In this section, let $R=\bigoplus_{n \geq 0} R_{n}$ be a Noetherian graded ring with $A=R_{0}$ a local ring with maximal ideal $m$. We always assume that $A$ is a quotient of a Gorenstein local ring.

For a complex of graded $R$-modules $C^{\circ}$, we denote $\left(C^{\circ}(n)\right)[p]$ the complex of graded $R$-modules defined by: $\left(\left(\left(C^{0}(n)\right)[p]\right)^{q}\right)_{m}=\left(C^{p+q}\right)_{n+m}$, where upper index means the degree as a complex and the lower index means the degree as graded modules. We always assume that the boundary homomorphisms of complexes of graded $R$-modules are homogeneous of degree 0 .

Our aim in this section is to describe the duality on $\operatorname{Proj}(R)$ in terms of the dualizing complex of $R$ which is a complex of graded $R$-modules.

Proposition (A.3.2). There exists a dualizing complex of $R$ which consists of graded $R$-modules.

Proof. By our assumption, $A$ has a dualizing complex. As $R$ is finitely generated over $A$, we can describe $R$ in the form: $R=S / J$, where $S$ is a polynomial ring over $A$ and $J$ a graded ideal of $S$. If we can prove that $S$ has a dualizing complex $D^{\circ}$ consisting of graded $S$-modules, then $\operatorname{RHom}_{S}\left(R, D^{\circ}\right)$ is a dualizing complex on $R$ consisted of graded $R$-modules. The existence of such $D^{*}$ is proved in the following lemma:

Lemma (A.3.3). Let $X$ be a Noetherian scheme which possesses a dualizing complex $D^{*}$, $L$ an invertible $O_{X}$-module, $B=\bigoplus_{n \geq 0} L^{n} T^{n}$ and $Y=\operatorname{Spec}_{X}(B) . \quad$ Then $\left(D^{\cdot} \otimes_{O_{X}}\left(\bigoplus_{n>0} L^{n} T^{n}\right)\right)$ is a dualizing complex of $Y$.

Proof. See [16], V.8.3.

Proposition (A.3.4). Let $D^{\circ}$ be a complex of graded $R$-modules in $D_{c}^{+}(R)$ (that is, $D^{p}=0$ for sufficiently small $p$ and $H^{q}\left(D^{\circ}\right)$ is finitely generated for every $q$ ), then $D^{\circ}$ is a dualizing complex if and only if there exist integers $d$ and $n$ for which the following conditions hold:

$$
\underline{\operatorname{Ext}}_{R}^{i}\left(\underline{A / m}, D^{\circ}\right)= \begin{cases}0 & \text { for } i \neq d, \\ (\underline{A / m})(n) & \text { for } i=d,\end{cases}
$$

where $A / m$ is the graded $R$-module whose component of degree 0 is $A / m$ and other components are 0 .

Proof. By [16], V.3.4, the complex $D^{*}$ is dualizing if and only if for every maximal ideal $\mathscr{M}$ of $R$ there exists an integer $d^{\prime}$ such that

$$
\underline{\operatorname{Ext}}_{R}^{i}\left(R / \mathscr{M}, D^{*}\right)= \begin{cases}0 & \text { for } i \neq d^{\prime} \\ R / \mathscr{M} & \text { for } i=d^{\prime}\end{cases}
$$

If $\mathscr{M}=R_{+}+m R$, the above isomorphism is contained in our assumption. If $\mathscr{M}$ is not homogeneous, let $\mathscr{P}$ be the maximal among homogeneous prime ideals contained in 
$\mathscr{M}$. Then, after the localization by homogeneous elements of $R-\mathscr{P}$, which does not affect $\operatorname{Ext}_{R}^{i}\left(R / \mathscr{M}, D^{\circ}\right)$, we may assume that the relation $\mathscr{M}=\mathscr{P}+t R$ holds for some $t \in \mathscr{M}-\mathscr{P}$ (cf. $\S 1$ of Chapter 1 of [12]). Now, by Lemma (A.3.5) below, $R / \mathscr{P}$ is dualizing with respect to $D^{\circ}$ and by the exact sequence

$$
0 \rightarrow R / \mathscr{P} \stackrel{t}{\rightarrow} R / \mathscr{P} \rightarrow \mathbb{R} / \mathscr{M} \rightarrow 0,
$$

we have the desired result.

Lemma (A.3.5). Under the assumption of Proposition (A.3.4), every graded $\mathbb{R}$-module $H$ is reflexive with respect to $D^{\circ}$. That is, the natural map $H \rightarrow \underline{\mathbb{R H o m}}_{R}\left(\underline{\mathbb{R H o m}}_{R}\left(H, D^{\circ}\right), D^{\circ}\right)$ is an isomorphism. Moreover, if $\mathscr{P}$ is a graded prime ideal of $R$, which does not contain $R_{+}$and $\operatorname{dim} R / \mathscr{P}=1$, then

$$
\left[\underline{\operatorname{Ext}}_{R}^{i}\left(R / \mathscr{P}, D^{\circ}\right)\right]_{(\mathscr{P})}= \begin{cases}0 & \text { for } i \neq d-1 \\ (R / \mathscr{P})_{(\mathscr{P})}(n) & \text { for } i=d-1\end{cases}
$$

Proof. The proof of the former part of our assertion goes exactly the same as [16], V.3.5. As $(R / \mathscr{P})_{(\mathscr{P})}$ is $H$-simple, every graded $(R / \mathscr{P})_{(\mathscr{P})}$-module is free (cf. [12], I, $\S 1)$. As $R / \mathscr{P}$ is reflexive with respect to $\mathbb{D}^{\circ}$, $\left[\operatorname{Ext}_{R}^{i}\left(R / \mathscr{P}, D^{\circ}\right)\right]_{(\mathscr{P})}$ equals $(R / \mathscr{P})_{(\mathscr{P})}$ if it is not zero. Taking a homogeneous element $t$ such that $P+t \mathbb{R}$ is $\left(R_{+}+m \mathbb{R}\right)$-primary, we see $i=d-1$.

Proposition (A.3.6). If $D^{\circ}$ and $D^{\prime \circ}$ are dualizing complexes of $\mathbb{R}$ both of which are complexes of graded $R$-modules, then they are isomorphic up to grading of complex and shift of gradings as $\mathbb{R}$-modules.

Proof. By [16], V.3.1, $D^{\circ}$ and $D^{\prime \circ}$ differ only by shift of gradings and multiplication by an invertible $R$-module $L$. On the other hand, $L$ equals $\underline{\operatorname{RHom}}\left(\mathbb{D}^{\circ}, \mathbb{D}^{\prime \circ}\right)$ up to shift of gradings. Hence $L$ is a graded $R$-module. Since $A=R_{0}$ is a local ring, $L$ is free.

Definition (A.3.7). A dualizing complex $D^{\circ}$ of $R$ consisted of graded $\mathbb{R}$-modules and graded homomorphism of degree zero is a normalized graded dualizing complex of $R$ if

$$
\underline{\operatorname{Ext}}_{R}^{i}\left(\underline{A / m}, D^{\circ}\right)= \begin{cases}0 & \text { for } i \neq 0 \\ \underline{A / m} & \text { for } i=0 .\end{cases}
$$

As we have seen, the normalized graded dualizing complex of $\mathbb{R}$ is unique up to isomorphism.

As usual, if $M$ is a graded $R$-module and if $d$ is a positive integer, we put $M^{(d)}=\bigoplus_{n \in \mathbb{Z}} M_{n d}$. If $C^{\circ}$ is a complex of graded $R$-modules, we put $\left(C^{\circ}\right)^{(d)}$ by: $\left(C^{\circ}\right)^{(d)}=$ $\left(\left(C^{p}\right)^{(d)}\right)_{p \in \mathbb{Z}}$.

Lemma (A.3.8). Let $\mathbb{D}_{R}^{\circ}$ be a normalized dualizing complex of $R, J$ a homogeneous ideal of $R$ and $d$ a positive integer.

(i) Then $\left(D_{R}^{\circ}\right)^{(d)}$ (resp. $\left.\underline{\mathbb{R H o m}}_{R}\left(R / J, D_{R}^{\circ}\right)\right)$ is a normalized dualizing complex of $\mathbb{R}^{(d)}$ (resp. $R / J)$. 
(ii) We have a canonical isomorphism

$$
D_{R}^{\circ} \neg \underline{\operatorname{RHom}}_{R^{(d)}}\left(R, D_{R^{(d)}}^{\circ}\right)
$$

for every integers $n$ and $d$ with $d>0$.

(iii) If we put $\omega_{X}(n)=\left(K_{R}(n)\right)^{\sim}$, then the canonical homomorphism

$$
\omega_{X}(n) \rightarrow \operatorname{Hom}_{O_{X}}\left(O_{X}(-n), \omega_{X}\right)
$$

is an isomorphism for every integer $n$.

Proof. (i) We can easily check the following relations

$$
\underline{\operatorname{Ext}}_{R^{(d)}}\left(\underline{A / m},\left(D_{R}^{\circ}\right)^{(d)}\right)=\left[\underline{\operatorname{Ext}}_{R}^{i}\left(\underline{A / m}, D_{R}^{*}\right)\right]^{(d)}= \begin{cases}0 & \text { for } i \neq 0 \\ A / m & \text { for } i=0\end{cases}
$$

And similarly for $R / J$. Thus we can conclude that $\left(D_{R}^{\circ}\right)^{(p)}\left(\operatorname{resp} . \underline{\mathrm{RHom}}_{R}\left(R / J, D_{R}^{\circ}\right)\right)$ is a normalized graded dualizing complex of $R^{(d)}$ (resp. $R / J$ ).

(ii) Since both sides are normalized dualizing complexes of $R$, twisted by degree $n$, they should be isomorphic.

(iii) From (ii) we have an isomorphism

$$
\left[D_{R}(n)^{\circ}\right]^{(d)} \rightarrow \underline{\mathbb{R H o m}}_{R^{(d)}}\left(R^{(-n, d)},\left(D_{R}^{\cdot}\right)^{(d)}\right) .
$$

If we take $d$ so that $R^{(d)}=R_{0}\left[R_{d}\right]$ and if we operate the functor ( $)^{\sim}$ of both sides and take cohomology of the lowest degree term, we get the desired isomorphism.

Theorem (A.3.9). If $D^{\circ}$ is a normalized graded dualizing complex of $R$, then $\left(D^{\circ}\right)^{\sim}=$ $\left(\left(D^{p}\right)^{\sim}\right)_{p \in \mathbb{Z}}$ is a dualizing complex on $X=\operatorname{Proj}(R)$. More precisely, for every closed point $x$ of $X$, we obtain the following relations:

$$
\operatorname{Ext}_{O_{X}}^{i}\left(k(x),\left(D^{\circ}\right)^{\sim}\right)= \begin{cases}0 & \text { for } \quad i \neq-1 \\ k(x) & \text { for } \quad i=-1\end{cases}
$$

Proof. We have only to show the isomorphism for Ext group as above. Now, a closed point $x$ of $X$ corresponds to a graded prime ideal $\mathscr{P}$ of $R$ which does not contain $R_{+}$and $\operatorname{dim} R / \mathscr{P}=1$. In this correspondence, $k(x)$ is the degree zero part of $(R / \mathscr{P})_{(\mathscr{P})}$. Then our assertion is the case $d=n=0$ of Lemma (A.3.5).

Remark (A.3.10). If $R=\bigoplus_{n \in \mathbb{Z}} R_{n}$ is a graded ring with both positive and negative graded parts and assume $R$ satisfies the following conditions;

(i) $R$ is finitely generated over a local ring $(A, m)$ contained in $R_{0}$, which is a quotient of a Gorenstein local ring.

(ii) $R$ has a unique graded maximal ideal $\mathscr{N}$ and the inclusion $A \subset R$ induces an isomorphism of $A / m$ and $R / \mathcal{N}$.

Then we can also prove the existence and uniqueness of a complex $D^{\circ}$ of graded $R$-modules with the property

$$
\underline{\operatorname{Ext}}_{R}^{i}\left(R / \mathcal{N}, D^{\circ}\right)= \begin{cases}0 & (i \neq 0) \\ R / \mathcal{N} & (i=0)\end{cases}
$$


in the same manner as in (A.3.6). We will call this $D^{\circ}$, also, the normalized dualizing complex of $R$.

Remark (A.3.11). Let $D^{\circ}$ be as in (A.3.7) or (A.3.10). We define

$$
K_{R}=H^{-d}\left(D^{*}\right)
$$

where $d=\operatorname{dim} R$ and call this module the canonical module of $R$. By (A.3.9) and the exactness of the functor ( $)^{\sim},\left(K_{R}\right)^{\sim}$ is a dualizing module on $X=\operatorname{Proj}(R)$ if $R$ is as in (A.3.1) (cf. also (A.3.12)).

Remark (A.3.12). Let $X$ be a Noetherian scheme with a dualizing complex $D^{\circ}$. Recall that $D^{\circ}$ is normalized if for every closed point $x$ of $X$.

$$
\underline{\operatorname{Ext}}_{o_{X}}^{i}\left(k(x), \mathbb{D}^{\circ}\right)= \begin{cases}0 & \text { for } i \neq 0 \\ k(x) & \text { for } i=0 .\end{cases}
$$

If $\operatorname{dim} X=d$ and if $D^{\circ}$ is a normalized dualizing sheaf on $X$, we define the dualizing sheaf $\omega_{X}$ of $X$ by $\omega_{X}=H^{-d}\left(D^{\circ}\right)$. By this definition, $\omega_{X}$ is defined up to tensor product of an invertible sheaf.

Proposition (A.3.13). Let $D^{\circ}$ be the normalized dualizing complex of $R$ and $\mathscr{N}$ the unique graded maximal ideal of $R$. Then there is a canonical isomorphism of graded R-modules

$$
H_{\mathcal{N}}^{i}(M) \simeq \underline{\operatorname{Hom}}_{A}\left(\underline{\operatorname{Ext}}_{R}^{-i}\left(M, D^{\circ}\right), \underline{E}_{A}(A / m)\right)
$$

for a finitely generated graded $R$-module $M$ and every $i$. In particular, we obtain the relation.

$$
H_{\mathcal{N}}^{d}(M) \neg \underline{\operatorname{Hom}}_{A}\left(K_{R}, \underline{E}(A / m)\right), \quad \text { where } \quad d=\operatorname{dim} A .
$$

This shows that our $K_{R}$ is the same module as is defined in [12] if $\mathbb{R}$ is positively graded and finitely generated over $R_{0}=k$, a field.

Proof. The same as in [16], V.6.2.

Remark (A.3.14). Let the situation be as in $\S 1$. Then our dualizing complex $D_{X}^{\circ}=\left(D_{R}^{\circ}\right)^{\sim}[-1]$, where $D_{R}^{\circ}$ is the normalized dualizing complex of $R$, is isomorphic to $\psi^{!}\left(D_{A}^{\circ}\right)$ in the notation of [16], Chapter $\mathbb{I I I}$ by our construction of $D_{R}^{\circ}$. So, by [16] III, 11.1 , for every coherent $O_{X}$-module $F$, we have the isomorphism

$$
\mathbb{R} \psi_{*}\left(\operatorname{RHom}_{X}\left(F, D_{X}^{*}\right)\right) \simeq \mathbb{R} \operatorname{Hom}_{A}\left(R \psi_{*} F, D_{A}^{*}\right) .
$$

(We identify a sheaf $G$ on $\bar{W}=\operatorname{Spec}(A)$ with $H^{0}(W, G)$.)

\section{§4. Criterion for Normality and Rational Singularity}

(4.1) In this section, we assume the following conditions on $A$ :

(4.1.1) $A$ is essentially of finite type over a field of characteristic 0.

(4.1.2) $\operatorname{Spec}(A)-\{m\}$ has only rational singularities (hence, is Cohen-Macaulay).

(4.1.3) $I=F^{N}$ (see (1.1)) is $m$-primary. That is, $X-E$ is isomorphic to $W-\{m\}$. 
Also, let $X^{\prime}$ be the normalization of $X$ and $\tilde{X}$ a desingularization of $X^{\prime}$, so that we have the following commutative diagram:

In this situation,

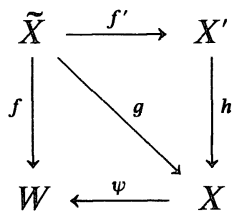

$H^{q}\left(\tilde{X}, O_{\tilde{X}}\right) \cong H_{m}^{q+1}(A)$ holds for $q$ with $0<q<d-1$, and

$H^{d-1}\left(\tilde{X}, O_{\tilde{X}}\right) \cong \operatorname{Hom}_{A}\left(\omega_{A} / f_{*}\left(\omega_{\tilde{X}}\right), E_{A}(A / m)\right)$, where $d=\operatorname{dim} A$ (cf. [8], [35], [51]). We define the geometric genus of the singularity $(A, m)$ (or of $(W, w)$ ) by the integer $p_{g}(A)=1_{A}\left(H^{d-1}\left(\tilde{X}, O_{\tilde{X}}\right)\right)=1_{A}\left(\omega_{A} / f_{*}\left(\omega_{\tilde{X}}\right)\right)$, where $1_{A}$ means the length as an $A$-module.

In particular, $A$ is a rational singularity if and only if $A$ is Cohen-Macaulay and $p_{g}(A)=0$ in our situation.

First, we shall prove the following:

Theorem (4.2). Assume one of the following conditions;

(i) $\left[H_{G_{+}}^{d-1}(G)\right]_{n}=0$ for every $n<0$.

(ii) $X$ has only rational singularities (in particular, $X$ is normal).

(iii) $H^{d-1}\left(C^{*} / F^{0}\left(C^{*}\right)\right)=0$ holds.

Then the inequality $p_{g}(A) \geq 1_{A}\left(H^{d-1}\left(X, O_{X}\right)\right)$ holds.

Furthermore if, in particular, $G$ is Cohen-Macaulay (resp. Gorenstein with $a(G)=a$ ), then we can describe the right hand side of the inequality above as follows:

$$
\begin{aligned}
1_{A}\left(H^{d-1}\left(X, O_{X}\right)\right) & =1_{A}\left(F^{0}\left(H_{m}^{d}(A)\right)\right)=\sum_{n \geq 0} 1_{A}\left(\left[H_{G_{+}}^{d}(G)\right]_{n}\right) \\
(\text { resp. } & =\sum_{0 \leq n \leq a} 1_{A}\left(G_{n}\right) \quad \text { (cf. [46])). }
\end{aligned}
$$

Proof. Let $\widetilde{E}$ be the scheme theoretic inverse image of $E$ in $\tilde{X}$. By our assumption on $A, H^{q}\left(\tilde{X}-\tilde{E}, O_{\tilde{X}}\right)=H^{q}\left(X-E, O_{X}\right)$ for every $q \geq 0$. If our condition (iii) holds, then the canonical map $H^{d-1}\left(X, O_{X}\right) \rightarrow H^{d-1}\left(X-E, O_{X}\right)$ is injective (see $\left.\S 1\right)$. Since this canonical map factors through $H^{d-1}\left(\tilde{X}, O_{\tilde{X}}\right)$, we have an injection $H^{d-1}\left(X, O_{X}\right) \rightarrow H^{d-1}\left(\tilde{X}, O_{\tilde{X}}\right)$. Condition (i) implies the condition (iii) by Lemma (1.18). By the following lemma, the condition (ii) implies the injectivity of the map $H^{d-1}\left(X, O_{X}\right) \rightarrow H^{d-1}\left(X-E, O_{X}\right)$.

Lemma (4.3). (i) If $X$ has only rational singularities, then the canonical map $H^{q}\left(X, O_{X}\right) \rightarrow H^{q}\left(X-E, O_{X}\right)$ is injective for every $q \geq 0$.

(ii) If $Y$ has only rational singularities, then $\left[H_{G_{+}}^{q}(G)\right]_{n}=0$ for every couple $(n, q)$ with $n<0$ and $q<d$.

(iii) Suppose that $X$ is normal and that $O_{X}(n)$ is $O_{X}$-invertible with the relation $O_{X}(n) \cong O_{X}(1)^{\otimes n}$ for every $n \in \mathbb{Z}$. Further we assume $O_{X}(n)$ is base point free for $n \geq 0$ or $\operatorname{dim} A=2$. Then $\left[H_{G_{+}}^{1}(G)\right]_{n}=0$ for every $n<0$.

Proof. (i) By Leray's spectral sequence and Grauert-Riemenschneider's vanishing theorem $([13]), 0=H^{i}\left(\tilde{X}, \omega_{\tilde{X}}\right)=H^{i}\left(X, g_{*}\left(\omega_{\tilde{X}}\right)\right)$ for $i>0$. Since $X$ has only rational sin- 
gularities, $H^{i}\left(X, \omega_{X}\right)=0$ for $i>0$. Hence, by the duality theorems (cf. (2.d) of [29]), $H_{E}^{d-i}\left(X, O_{X}\right)=0$ for $i>0$, because $X$ is Cohen-Macaulay. Therefore our assertion holds.

(ii) By similar argument as above, we obtain $H^{i}\left(Y, \omega_{Y}\right)=0$ for $i>0$. Hence $H^{i}\left(X, \omega_{X}(n)\right)=0$ for every $i>0$ and $n>0$ by Proposition (3.2). Therefore $H_{E}^{d-i}\left(X, O_{X}(-n)\right)=0$ for $i>0$ and $n>0$ by the duality theorems as above and (iii) of Lemma (A.3.8). Our assertion follows by the exact sequence (1.18.1) and the vanishings $H^{i}\left(C^{\circ} / F^{n}\left(C^{\circ}\right)\right)=0$ for $i=0,1$ and $n \leq 0$.

(iii) Put $O_{\tilde{X}}(n)=g^{*}\left(O_{X}(n)\right)$ for every $n$. By the Grauert-Riemenschneider vanishing ([13], see also [29]), $R^{r} f_{*}\left(\omega_{\tilde{X}} \otimes_{o_{\tilde{X}}} O_{\tilde{X}}(n)\right)=0$ for $r>0$ and $n \geq 0$. By duality theorems (cf. (2.d) of [29]), $H_{\tilde{E}}^{d-r}\left(\tilde{X}, O_{\tilde{X}}(-n)\right)=0$ for $r>0$ and $n \geq 0$. By the Leray spectral sequence

$$
H_{E}^{p}\left(X, R^{q} g_{*}\left(O_{\tilde{X}}(n)\right)\right) \Rightarrow H_{E}^{p+q}\left(\tilde{X}, O_{\tilde{X}}(n)\right),
$$

$H_{E}^{1}\left(X, O_{X}(n)\right)=0$ for $n \leq 0$. The rest of proof goes as in (ii).

Corollary (4.4) Let $q$ be an integer with $0<q<d-1$.

(i) If $\left[H_{G_{+}}^{q}(G)\right]_{n}=0$ for every $n<0$, then the canonical map $\mathbb{H}^{q}\left(X, O_{X}\right) \rightarrow H^{q}\left(X^{\prime}, O_{X^{\prime}}\right)$ is injective.

(ii) Furthermore suppose $O_{X}(n)$ is invertible for every $n \in \mathbb{Z}$ and put $O_{X^{\prime}}(n)=h^{*}\left(O_{X}(n)\right)$ for every $n \in \mathbb{Z}$. Then the canonical map $H^{q}\left(X, O_{X}(n)\right) \rightarrow H^{q}\left(X^{\prime}, O_{X^{\prime}}(n)\right)$ is injective for every $n<0$.

Proof. By (iv) of Proposition (1.17), the canonical map $H^{q}\left(\mathbb{X}, O_{X}(n)\right) \rightarrow$ $H^{q}\left(X-E, O_{X}(n)\right)$ is injective for $n \leq 0$. Since $X-\mathbb{E}$ is normal, this map factors through $H^{q}\left(X^{\prime}, O_{X^{\prime}}(n)\right)$ when $O_{X}(n)$ is invertible.

Corollary (4.5). Assume that $A$ is a Cohen-Macaulay ring in the situation (4.1). Consider the following three conditions.

(a) $\left[H_{G_{+}}^{d-1}(G)\right]_{n}=0$ holds for every $n<0$.

(b) $X$ has only rational singularities.

(c) $H_{G_{+}}^{d-1}(G)=0$ holds.

Then: (i) Suppose that $A$ is a rational singularity and that (a) holds. Then $a(A)<0$ in the sense of (1.15).

(ii) Suppose (b) holds. Then $A$ is a rational singularity if and only if $\mathbb{H}^{d-1}\left(X, O_{X}\right)=$ $F^{0}\left(H_{m}^{d}(A)\right)=0$.

(iii) Suppose (b) and (c) hold. Then $A$ is a rational singularity if and only if $a(A)=a(G)<0(c f .(1.15))$.

This is a direct consequence of Theorem (4.2).

Now, we shall seek conditions for $X$ to be normal or to have only rational singularities in terms of numerical data which relates to the geometric genus $p_{g}(A)$. First we shall treat the case $d=\operatorname{dim} A=2$ in the following:

Theorem (4.6). Assume that $A$ is normal with $\operatorname{dim} A=2$ and that the following conditions hold for $A$ : 
(a) $\left[H_{G_{+}}^{1}(G)\right]_{n}=0$ for $n \leq 0$.

(b) $O_{X}(n)$ is invertible $O_{X}$-module and $O_{X}(n)=\left(O_{X}(1)\right)^{\otimes n}$ for every $n \in \mathbb{Z}$.

(c) $F^{1}=m$.

Then: (i) $X$ is normal if and only if the relation $1_{A}\left(H^{1}\left(X^{\prime}, O_{X^{\prime}}\right)\right)=1_{A}\left(H^{1}\left(X, O_{X}\right)\right)$ holds.

(ii) $X$ is normal and has only rational singularities if and only if the relation $p_{g}(A)=1_{A}\left(H^{1}\left(X, O_{X}\right)\right)$ holds.

Proof. (i) We shall show $X=X^{\prime}$ from the condition $1_{A}\left(H^{1}\left(X, O_{X}\right)\right)=$ $1_{A}\left(H^{1}\left(X^{\prime}, O_{X^{\prime}}\right)\right)$ in the below.

Define an $O_{X}$-module $N$ by the exact sequence:

$$
0 \rightarrow O_{X} \rightarrow h_{*}\left(O_{X^{\prime}}\right) \rightarrow N \rightarrow 0,
$$

and denote $N \otimes O_{X}(n)$ by $N(n)$. Then the support of $N(n)$ is contained in $E$, which is a projective scheme of dimension one over $A / m$. So, $\chi(N(n))=1_{A}\left(H^{0}(X, N(n))\right)-$ $1_{A}\left(H^{1}(X, N(n))\right)$ is a polynomial of degree one. Let us set $P(n)=\chi(N(n))=a_{1} n+a_{0}$ with integers $a_{1}$ and $a_{0}$ for $n \in \mathbb{Z}$. Note that $a_{1} \geq 0$ holds as $O_{X}(1)$ is ample on E. Since $H^{0}\left(X, O_{X}\right)=H^{0}\left(X^{\prime}, O_{X^{\prime}}\right)=A$ and $H^{1}\left(X, O_{X}\right) \rightarrow H^{1}\left(X^{\prime}, O_{X^{\prime}}\right)$ is bijective by Corollary (4.4) and our assumption, we obtain the relation $P(0)=a_{0}=0$. For $a_{1}$, we see similarly the followings: $H^{0}\left(X, O_{X}(1)\right)=H^{0}\left(X^{\prime}, O_{X^{\prime}}(1)\right)=m$ and the injectivity of $H^{1}\left(X, O_{X}(1)\right) \rightarrow H^{1}\left(X^{\prime}, O_{X^{\prime}}(1)\right)$ follow by Proposition (1.17), Corollary (4.4) and our assumption (c). These imply the relations $P(1)=a_{1}=-1_{A}\left(H^{1}(X, N(1)) \leq 0\right.$. Hence $P(n)=0$ for every $n \in \mathbb{Z}$. This implies the assertion $N=0$. The converse implication is trivial.

(ii) Assume that the relation $\mathbb{1}_{A}\left(H^{1}\left(X, O_{X}\right)\right)=p_{g}(A)$ holds. Then $1_{A}\left(H^{1}\left(X, O_{X}\right)\right)=$ $1_{A}\left(H^{1}\left(X^{\prime}, O_{X^{\prime}}\right)\right)$ holds by Corollary (4.4). Hence $X$ is normal by (i). By Leray's spectral sequence, we have the exact sequence:

$$
0 \rightarrow H^{1}\left(X, O_{X}\right) \rightarrow H^{1}\left(\tilde{X}, O_{\tilde{X}}\right) \rightarrow H^{0}\left(X, R^{1} g_{*}\left(O_{\tilde{X}}\right)\right) \rightarrow 0 .
$$

Our assumption implies that $R^{1} g_{*}\left(O_{\tilde{X}}\right)=0$, which means that $X$ has only rational singularities as desired. The converse implication is trivial.

Q.E.D.

To prove a theorem generalizing Theorem (4.6) to arbitrary dimension, we shall rewrite Theorem (4.2) and Corollary (4.4) in terms of dualizing sheaves. Here we denote by $\omega_{V}$ the dualizing sheaf on a scheme $V$. We can choose the dualizing module as "Zariski sheaf" with the property (see the carefull treatment in the article [30]): If we restrict the dualizing sheaves $\omega_{W}, \omega_{X}$, and $\omega_{X^{\prime}}$ to $X-E$, they are isomorphic by the morphisms induced canonically. Further $g_{*}\left(\omega_{\tilde{X}}\right) \rightarrow \omega_{X}$ gives the isomorphism on $X-$ E. ... etc.

Proposition (4.7). There are canonical inclusions, $g_{*}\left(\omega_{\tilde{X}}\right) \subset h_{*}\left(\omega_{X^{\prime}}\right) \subset \omega_{X}$, and, consequently,

$$
p_{g}(A)=1_{A}\left(\omega_{W} / f_{*}\left(\omega_{\tilde{X}}\right)\right) \geq 1_{A}\left(\omega_{W} /(\psi \cdot h)_{*}\left(\omega_{X^{\prime}}\right)\right) \geq 1_{A}\left(\omega_{W} / \psi_{*}\left(\omega_{X}\right)\right) .
$$

Moreover, if $\left[H_{G_{+}}^{d-1}(G)\right]_{n}=0$ for every $n<0$, then the relation $1_{A}\left(\omega_{W} / \psi_{*}\left(\omega_{X}\right)\right)=$ $1_{A}\left(H^{d-1}\left(X, O_{X}\right)\right)$ holds. 
Proof. Since $X^{\prime}$ is normal, $\left(f^{\prime}\right)_{*}\left(\omega_{\tilde{X}}\right) \subset \omega_{X^{\prime}}$ follows. Further we have the canonical inclusions, as desired, by the relation $h_{*}\left(\omega_{X^{\prime}}\right)=\operatorname{Hom}_{O_{X}}\left(O_{X^{\prime}}, \omega_{X}\right)$. Our additional assumption implies that the canonical map $H^{d-1}\left(X, O_{X}\right) \rightarrow H^{d-1}\left(X-E, O_{X}\right)$ is injective. By the duality theorems (cf. (2d) of [29]), $\omega_{W} / \psi_{*}\left(\omega_{X}\right)$ is dual to the $A$-module $\operatorname{Ker}\left\{H^{d-1}\left(X-E, O_{X}\right) \rightarrow H_{E}^{d}\left(X, O_{X}\right)\right\}$, which is isomorphic to $H^{d-1}\left(X, O_{X}\right)$ Q.E.D.

Proposition (4.8). Assume that $G$ is Cohen-Macaulay and $\omega_{X}$ is generated by $H^{0}\left(X, \omega_{X}\right)$ as $O_{X}$-module. Then:

(i) $X$ is normal if and only if the relation

holds.

$$
\begin{aligned}
1_{A}\left(H^{d-1}\left(X, O_{X}\right)\right)( & \left.=\sum_{0 \leq n \leq a(G)} 1_{A}\left(\left[H_{G_{+}}^{d}(G)\right]_{n}\right)\right) \\
& =1_{A}\left(\omega_{W} /(\psi \cdot h)_{*}\left(\omega_{X^{\prime}}\right)\right)
\end{aligned}
$$

(ii) $X$ is normal and has only rational singularities if and only if

holds.

$$
p_{g}(A)=1_{A}\left(H^{d-1}\left(X, O_{X}\right)\right)
$$

Proof. (i) By Proposition (4.7),

$$
1_{A}\left(H^{d-1}\left(X, O_{X}\right)\right)=1_{A}\left(\omega_{W} /(\psi \cdot h)_{*} \omega_{X^{\prime}}\right)
$$

holds if and only if $\psi_{*}\left(\omega_{X}\right)=\psi_{*}\left(h_{*}\left(\omega_{X^{\prime}}\right)\right)$ holds. In this case, we have the following commutative diagram:

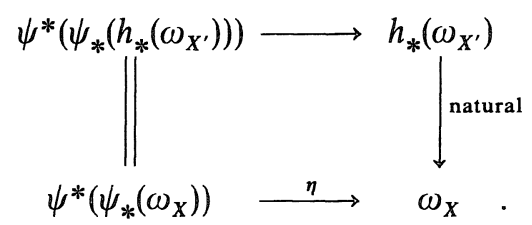

By our assumption, $\eta$ is surjective. Hence the relation $h_{*}\left(\omega_{X^{\prime}}\right)=\omega_{X}$ follows. Since $X$ is Cohen-Macaulay,

$$
O_{X}=\operatorname{Hom}_{O_{X}}\left(\omega_{X}, \omega_{X}\right)=\operatorname{Hom}_{O_{X}}\left(h_{*}\left(\omega_{X^{\prime}}\right), h_{*}\left(\omega_{X^{\prime}}\right)\right) \supset h_{*}\left(\operatorname{Hom}_{O_{X^{\prime}}}\left(\omega_{X^{\prime}}, \omega_{X^{\prime}}\right)\right)=h_{*}\left(O_{X^{\prime}}\right) .
$$

Therefore $X$ is normal. The converse implication is trivial.

(ii) Suppose the relation $p_{g}(A)=1_{A}\left(H^{d-1}\left(X, O_{X}\right)\right)$ holds. Then, by Proposition (4.7), $\psi_{*}\left(\omega_{X}\right)=\psi_{*}\left(h_{*}\left(\omega_{X^{\prime}}\right)\right)=\psi_{*}\left(g_{*}\left(\omega_{\tilde{X}}\right)\right)$. By the proof of (i), $X$ is normal. Again we have the following commutative diagram:

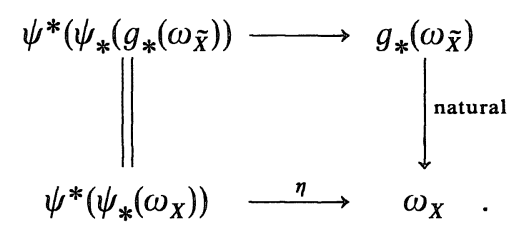

Since $\eta$ is surjective, $g_{*}\left(\omega_{\tilde{X}}\right)=\omega_{X}$. Therefore $X$ has only rational singularities, because $X$ is Cohen-Macaulay (see pp. 50-51 of [24]).

Q.E.D. 
Theorem (4.9)*. Let us assume that $G$ is Cohen-Macaulay and that one of following two conditions holds:

(a) $G$ is Gorenstein with $a(G) \geq-1$.

(b) $K_{G}$ is generated by the elements of degree $\leq 1$ as $G$-module. Then the relations $p_{g}(A)=1_{A}\left(H^{d-1}\left(X, O_{X}\right)\right)=\sum_{0 \leq n \leq a(G)} 1_{A}\left(\left[H_{G_{+}}^{d}(G)\right]_{n}\right)$ hold if and only if $X$ is normal and has only rational singularities.

Proof. This follows from (4.8), (i) of (3.5) and (3.9).

We apply Theorem (4.9) to the following situation.

(4.10). Let $A$ be the local ring of $d$-dimensional hypersurface isolated singularity of multiplicity $\rho$ written as follows: $A=k\left[\left[x_{0}, x_{1}, \ldots, x_{d}\right]\right] /(f), f \in S=k\left[\left[x_{0}, \ldots, x_{d}\right]\right]$, and $f=x_{0}{ }^{\rho}+\sum_{i=1}^{\rho} g_{i}\left(x_{1}, \ldots, x_{d}\right) x_{0}{ }^{\rho-i}$ with $g_{i}\left(x_{1}, \ldots, x_{d}\right) \in\left(x_{1}, \ldots, x_{d}\right)^{i}$ for $i=1, \ldots, \rho$. Let $r$ be the integer defined by $r=\min _{1 \leq i \leq \rho}\left[\frac{\text { ord } g_{i}}{i}\right](\geq 1)$. Let us introduce a filtration $\left\{F^{k}(S)\right\}_{k \geq 0}$ on $S$ by putting weights on each monomials as follows; wt $\left(x_{0}\right)=r$ and $\operatorname{wt}\left(x_{i}\right)=1$ for $1 \leq i \leq d$. We shall introduce the filtration $\left\{F^{k}(A)\right\}_{k \geq 0}$ on $A$ by; $F^{k}(A)=$ $F^{k}(S) A$ for $k \geq 0$. Then we can easily see that $G=\bigoplus_{k \geq 0} F^{k} / F^{k+1}$ is a hypersurface of the form $k\left[x_{0}, x_{1}, \ldots, x_{d}\right] /\left(f_{r \rho}\right)$, where $f_{r \rho}$ is a weighted homogeneous polynomial of degree $r \rho$. By [12], we have $a(G)=r(\rho-1)-d$. Now we obtain the following:

Corollary (4.11)*. Let $(A, m)$ be the local ring of a d-dimensional hypersurface isolated singularity of multiplicity $\rho$ as in (4.10). Then

(i) We have the following inequalities

$$
p_{g}(A) \geq \sum_{0 \leq s \leq \rho-1}\left(\begin{array}{l}
r s \\
d
\end{array}\right) \geq\left(\begin{array}{c}
\rho \\
d+1
\end{array}\right)
$$

where the binomial coefficient $\left(\begin{array}{l}j \\ i\end{array}\right)$ means 0 for $j<i$.

(ii) Furthermore assume the relation $r(\rho-1) \geq d-1$. Then the equality holds in the first inequality of (i) if and only if $X=\operatorname{Proj}\left(\underset{k \geq 0}{\oplus} F^{k}\right)$ is normal and has only rational
singularities.

(iii) Assume the relation $\rho \geq d$. Then the equality $p_{g}(A)=\left(\begin{array}{c}\rho \\ d+1\end{array}\right)$ holds if and only if the blowing-up of $A$ with center maximal ideal is normal and has only rational singularities.

Proof. Let the notations be as in (4.10). Since $a(G)<r \rho=\operatorname{deg}\left(f_{r \rho}\right)$, we have $\sum_{0 \leq k \leq a(G)} 1_{A}\left(G_{k}\right)=\sum_{0 \leq k \leq a(G)} \operatorname{dim}\left(F^{k}(S) / F^{k+1}(S)\right)$. Hence (i) and (ii) follow from (4.2), (4.9) and (4.10). For (iii), apply the arguments above for the maximal-ideal-adic filtration of $A$.

Remark (4.12). This corollary is a generalization of Theorem (3.14) of [26] and Theorem (1.3) of [50].

* See Note Added in Proof (1). 
Example (4.13). (i) Let $A$ be the local ring of the singularity $\left\{x^{3}+y^{a}+z^{b}=0\right\}$ in $k^{3}$ at the origin, where $a$ and $b$ are integers with $3 \leq a \leq b$. Then the blowing-up $X$ of $A$ with center maximal ideal $m$ is normal and has only rational singularities if and only if $(a, b)$ is one of followings: $(3,3),(3,4),(3,5)$ and $(4,4)$.

(ii) Let $A$ be the local ring of the singularity $\left\{x^{4}+y^{a}+z^{b}+w^{c}=0\right\}$ in $k^{4}$ at the origin, where $a, b$ and $c$ are integers with $4 \leq a \leq b \leq c$. Then the blowing-up $X$ of $A$ with center maximal ideal $m$ is normal and has only rational singularities if and only if $(a, b, c)$ is one of followings: $(4,4, c)$ with $4 \leq c \leq 7,(4,5,5),(4,5,6)$ and $(5,5,5)$.

Proof. In these cases, one can easily see that $G=\bigoplus_{k \geq 0} m^{k} / m^{k+1}$ is Gorenstein with $a(G)=0$. Hence $X$ has only rational singularities if and only if $p_{g}(A)=1$. For (i), by using the fact that $A$ is quasi-homogeneous with $\operatorname{deg}(x)=a b, \operatorname{deg}(y)=3 b$ and $\operatorname{deg}(z)=3 a$, we can see that $p_{g}(A)=1$ if and only if $3 a b-a b-3 b-3 a<3 a$ (cf. [46] (2.13)). For (ii), by using the fact that $A$ is quasi-homogensous with $\operatorname{deg}(x)=a b c$, $\operatorname{deg}(y)=4 b c, \operatorname{deg}(z)=4 c a$, and $\operatorname{deg}(w)=4 a b, p_{g}(A)=1$ if and only if $4 a b c-a b c-$ $4 b c-4 c a-4 a b<4 a b$.

\section{§5. The Case Whem $\mathbb{G}$ Has man}

(5.1) We always keep the notation of Section 1. Moreover, in this section, we assume that $(A, m)$ is a normal local ring containing a field $k, \mathbb{I}$ is $m$-primary and that $G$ is an integral domain. We assume there is no integer $r \geq 2$ with $G^{(r)}=G$. Also these assumptions imply that $\mathscr{R}$ is normal and we have $F^{k}=\psi_{*}\left(O_{X}(k)\right)=\psi_{*}\left(O_{X}(-k \cdot E)\right)$ by (iii) of Proposition (1.6) and the arguments of (2.2). In the first part of this section, our purpose is to prove Theorem (5.6). In the second part, we will show that our space $X$ is a deformation of $\operatorname{Proj}\left(G^{\natural}\right)$ (Proposition (5.17)). In the case $\operatorname{dim} A=2$, this implies that the canonical partial resolution of a singularity with "star-shaped" resolution is a deformation of that of a quasi-homogeneous singularity (cf. Corollary (5.18)).

Let $\bar{G}$ be the integral closure of $G$ in the quotient field of $G$. We note the following lemma for a graded ring over a field.

Lemma (5.2). Let $G=\bigoplus_{n \geq 0} G_{n}$ be a Noetherian graded ring with $\operatorname{dim} G \geq 2$ which is an integral domain with $G_{0}=k$, a field. Then the following conditions are equivalent to each other.

(i) $\operatorname{Spec}(G)-V\left(G_{+}\right)$is normal.

(ii) $\bar{G} / G$ has finite length.

(iii) $\bigoplus_{k \in \mathbb{Z}} H^{0}\left(E, O_{E}(k)\right)$ is normal, where $E=\operatorname{Proj}(G)$.

If these equivalent conditions hold, then $\bar{G} / G$ is isomorphic to $H_{G_{+}}^{1}(G)$.

Proof. We have (§5 of [23])

$$
0 \rightarrow G \rightarrow \bigoplus_{k \in \mathbb{Z}} H^{0}\left(E, O_{E}(k)\right) \rightarrow H_{G_{+}}^{1}(G) \rightarrow 0 .
$$

Since $E$ is reduced and irreducible with $\operatorname{dim} E \geq 1, \bigoplus_{k \in \mathbb{Z}} H^{0}\left(E, O_{E}(k)\right)$ is also an integral domain which is finitely generated over $k=G_{0}$. Hence $H_{G_{+}}^{1}(G)$ has finite length. 
If we assume (i), then $\bar{G} / G$ is a finite $G$-module supported by $V\left(G_{+}\right)$. Hence $\bar{G} / G$ has finite length. We get the converse implication by localizing by a homogeneous element of $G_{+}$.

If we assume these equivalent conditions (i) and (ii), we obtain the relations $O_{E}(k)=$ $G(k)^{\sim}=\bar{G}(k)^{\sim}$ on $E=\operatorname{Proj}(G)=\operatorname{Proj}(\bar{G})$ for $k \in \mathbb{Z}$. By the exact sequence being above, the relations $\bar{G}=\bigoplus_{k \in \mathbb{Z}} H^{0}\left(E, O_{E}(k)\right)$ and $\bar{G} / G=H_{G_{+}}^{1}(G)$ follow.

Next suppose the condition (iii). Since the length of $H_{G_{+}}^{1}(G)$ is finite, $\bigoplus_{k \in \mathbb{Z}} H^{0}\left(E, O_{E}(k)\right)$ is integral over $G$. Therefore $\bar{G}=\bigoplus_{k \in \mathbb{Z}} H^{0}\left(E, O_{E}(k)\right)$. The condition (ii) follows. Q.E.D.

Corollary (5.3). Let the situation be as in (5.1). If $E$ is a normal Cartier divisor on $X$, then $\bar{G} / G$ has finite length.

Proof. By the exact sequence of T.N.-isomorphisms;

$$
0 \rightarrow \mathscr{R}(n+1) \rightarrow \mathscr{R}(n) \rightarrow G(n) \rightarrow 0,
$$

$O_{X}(n) / O_{X}(n+1) \cong O_{E}(n)$ for $n \in \mathbb{Z} . \quad$ By the isomorphism $O_{X}(n) \cong O_{X}(-n \cdot E) \cong O_{X}(-E)^{\otimes n}$, we see that $O_{E}(n)$ is invertible and isomorphic to $O_{E}(1)^{\otimes n}$ for every integer $n$. Since $E$ is normal, so is $\bigoplus_{k \in \mathbb{Z}} H^{0}\left(E, O_{E}(k)\right)$.

Remark (5.4). There is an example with non-singular Weil divisor $E$ and infinite length $\bar{G} / G$ (see Example (2.5), and Example (6.19)).

Remark (5.5). Under the equivalent conditions of Lemma (5.2), we represent $\bar{G}$ by Demazure's construction [7]: $\bar{G}=R(E, D)$ with ample $\mathbb{Q}$-Weil divisor $D$ on $E$. The correspondence between $R(E, D)$ and $A$ in the exact sequence

$$
0 \rightarrow G \rightarrow R(E, D) \rightarrow H_{G_{+}}^{1}(G) \rightarrow 0
$$

is fundamental in Chapter 2. Taking the long exact cohomology sequence of

$$
0 \rightarrow O_{X}(k+1) \rightarrow O_{X}(k) \rightarrow O_{E}(k) \rightarrow 0
$$

for $k \in \mathbb{Z}$, we often represent $H_{G_{+}}^{1}(G)$ as $G$-module $U$ as follows:

$$
U=\bigoplus_{k \geq 0} \operatorname{Ker}\left\{R^{1} \psi_{*}\left(O_{X}(k+1)\right) \rightarrow R^{1} \psi_{*}\left(O_{X}(k)\right)\right\} .
$$

Further we can easily show the equality $1_{A}(U)=\sum_{k \geq 1}(-1)^{k+1}\left\{1_{A}\left(\left.H_{G_{+}}^{k+1}(G)\right|_{0}\right)-\right.$ $\left.1_{A}\left(R^{k} \psi_{*}\left(O_{X}\right)\right)\right\}$, where $\left.M\right|_{0}$ denotes the non-negative part for a graded module $M$.

Now, we will state the theorem which is our aim in the first half of this section. Although this result was treated by Flenner [9], our statement is not mentioned explicitly there and we also want to clarify the role of our spaces $Y$ and $Y^{\prime}$ there.

Theorem (5.6). Assume that $G$ is an integral domain and both $\operatorname{Spec}(A)-\{m\}$ and $\operatorname{Spec}(G)-V\left(G_{+}\right)$are regular (resp. have at most rational singularities), then $X$ has at most "cyclic quotient singularities" (resp. rational singularities). Moreover, if $\operatorname{dim} A=2$, then $A$ has a "star-shaped" resolution. 
Here, the definition of a "star-shaped" resolution is given in (0.5) and the definition of a "cyclic quotient" singularity (especially, in characteristic $p>0$ ) is given below. Also, if we mention a "rational singularity", we assume that char $(k)=0$ and that $A$ is essentially of finite type over $k$.

Definition-Proposition (5.7). Let $(B, m)$ be a d-dimensional Noetherian local ring which contains a field $k=B / m$ and $N_{B}$ a positive integer. Then the following conditions are equivalent to each other.

(i) There is a normal subsemigroup $H$ of $\mathbb{N}^{d}$ defined by

$$
H=\left\{\left(s_{1}, \ldots, s_{d}\right) \in \mathbb{N}^{d} \mid \sum_{i=1}^{d} a_{i} \cdot s_{i} \equiv 0\left(\bmod N_{B}\right)\right\}
$$

for some $d$-tuple $\left(a_{1}, \ldots, a_{d}\right)$ of integers such that the m-adic completion $B^{\wedge}$ is isomorphic to $k\left[\left[T_{1}^{s_{1}} \cdots T_{d} s_{d} \mid\left(s_{1}, \ldots, s_{d}\right) \in H\right]\right]$. Further each $T_{i}$ is an indeterminate over $k$ and is integral over $B$, for $1 \leq i \leq d$.

(ii) There is a regular overring $\left(C, m^{*}\right)$ of $B$ such that there is a grading $C=\bigoplus_{n=0}^{N_{B}-1} C_{n}$ by $\mathbb{Z} / N_{B} \mathbb{Z}$ on $C$ such that $C_{n} \subset m^{*}$ for $n=1, \ldots, N_{B}-1$ and $C_{0}=B$.

We say that $B$ is a cyclic quotient singularity if $B$ satisfies these equivalent conditions. of course, if $N_{B}$ is prime to char $(k)$, then $B$ is an invariant subring of a regular local ring by a cyclic group $\mathbb{Z} / N_{B} \mathbb{Z}$.

Proof. If we assume (i), put $C^{\wedge}=k\left[\left[T_{1}, \ldots, T_{d}\right]\right]$ and $C=B\left[T_{1}, \ldots, T_{d}\right]$. Then $C^{\wedge}$ (resp. $C$ ) is certainly a $\mathbb{Z} / N_{B} \mathbb{Z}$-graded ring with $\left(C^{\wedge}\right)_{0}=B^{\wedge}$ (resp. $C_{0}=B$ ). Since $C$ contains $T_{1}, \ldots, T_{d}, C^{\wedge}$ is the completion of $C$. So, $C$ is a regular local ring and we have our condition (ii).

If we assume the condition (ii), then $m^{*}=m+\bigoplus_{n=0}^{N_{B}-1} C_{n}$ and we can take a homogeneous element $f$ which is not contained in $\left(m^{*}\right)^{2}$. Repeating this process in $C / f \cdot C$ and so on, we can claim that we can take a regular parameter system $\left(t_{1}, \ldots, t_{d}\right)$ of $C$ consisting of homogeneous elements. Then the completion of $C$ is $k\left[\left[t_{1}, \ldots, t_{d}\right]\right]$ and we have the condition (i).

The proof of Theorem (5.6) is divided into several parts.

(5.8) Let $f \in F^{m}-F^{m+1}$ and put $f^{*}=f \cdot T^{m} \in \mathscr{R}_{m}$ as in $\S 1$. Then $u=T^{-1}$ is a non-zero divisor on $\mathscr{R}_{f^{*}}=\mathscr{R}_{f^{*}}^{\prime}$ and we obtain the following exact sequence

$$
0 \longrightarrow \mathscr{R}_{f^{*}}^{\prime} \stackrel{T^{-1}}{\longrightarrow} \mathscr{R}_{f^{*}}^{\prime} \rightarrow G_{\bar{f}} \longrightarrow 0 \text {. }
$$

Here we have the relation

$$
\left.O_{Y^{\prime}}\right|_{D_{+}\left(f^{*}\right)}=\left.\bigoplus_{k \in \mathbb{Z}} O_{X}(k)\right|_{D_{+}\left(f^{*}\right)} \cong\left(\mathscr{R}_{f^{*}}^{\prime}\right)^{\sim}
$$

Lemma (5.8.2). Under the assumption of Theorem (5.6), $Y^{\prime}$ is regular (resp. has at most rational singularities).

Proof. Here $\mathscr{R}_{f^{*}} / u \mathscr{R}_{f^{*}}=G_{\bar{f}}$ is regular (resp. has rational singularities) by our 
assumptions. So $\mathscr{R}_{f^{*}}$ is regular (resp. has rational singularities by Elkik [8]) at the prime ideals containing $u$, and, since $\left(\mathscr{R}_{f^{*}}\right)_{u}=\mathscr{R}_{f^{*}}\left[u^{-1}\right]=A_{f}\left[T, T^{-1}\right]$, so does $\mathscr{R}_{f^{*}}$ at the prime ideals not containing $u$.

(5.8.3) For our claim in (5.6) concerning rational singularities, we can finish our proof using Boutot's theorem, stating that a direct summand of a rational singularity is a rational singularity (cf. [4], [46]), because $O_{X}$ is a direct summand of $O_{Y^{\prime}}$.

(5.9) We are interested in the singularities of $X=\operatorname{Proj}(\mathscr{R})$. For this investigation, the integer $N$ such that $O_{X}(N)$ is invertible plays an essential role. The integer $N$ we have defined in (1.1) has such a property at any point of $X$. Since we study locally on $X$, our minimal positive integer $n$ with $O_{X}(n)$ invertible may be smaller than our $N$ of (1.1).

Let $x$ be a point of $E \subset X$ and $P$ (resp. $\bar{P}$ ) the homogeneous prime ideal of $\mathscr{R}$ (resp. $G$ ) associated to $x$. At the point $x=V(P)$, we note the following:

Lemma (5.9.1). Let the situation be as in the above. Then, the integers $N(X, x)=\min \left\{\alpha \in \mathbb{Z} \mid \alpha>0\right.$ and $O_{X}(\alpha)_{x} \cong\left(\mathscr{R}_{(P)}\right)_{\alpha}$ contains a unit of $\left.\mathscr{R}_{(P)}\right\}$, $N(E, x)=\min \left\{\alpha \in \mathbb{Z} \mid \alpha>0\right.$ and $O_{E}(\alpha)_{x} \cong\left(G_{(\bar{P})}\right)_{\alpha}$ contains a unit of $\left.G_{(P)}\right\}$, $N(P)=$ G.C.D. $\left\{n \in \mathbf{Z} \mid n>0\right.$ and $\left.(\mathscr{R} / P)_{n} \neq 0\right\}$, and

$N(\bar{P})=$ G.C.D. $\left\{n \in \mathbb{Z} \mid n>0\right.$ and $\left.(G / \bar{P})_{n} \neq 0\right\}$

are the same. Here $\mathscr{R}_{(P)}$ (resp. $G_{(\bar{P})}$ ) denotes the localization by all the homogeneous elements of $\mathscr{R}$ (resp. G) not contained in $P$ (resp. $\bar{P}$ ).

Proof. Being the homogeneous localization at the homogeneous prime $P, \mathscr{R}_{(P)}$ has the unique homogeneous maximal ideal $P \cdot \mathscr{R}_{(P)}$ and $\mathscr{R}_{(P)} / P \cdot \mathscr{R}_{(P)}=(\mathscr{R} / P)_{(P)} \cong k(x)\left[t, t^{-1}\right]$, where $k(x)=\left(\mathscr{R}_{(P)} / P \cdot \mathscr{R}_{(P)}\right)_{0}=\left(G_{(\bar{P})} / \bar{P} \cdot G_{(\bar{P})}\right)_{0}$ and $t$ is homogeneous of degree $N(P)$ (cf. Chapter 1 of [12]). This shows the equality $N(X, x)=N(P)$. By the same arguments for $G_{(\bar{P})}=\mathscr{R}_{(P)} / T^{-1} \mathscr{R}_{(P)}$, we obtain the equality $N(E, x)=N(\bar{P})$. Here we have the relation $T^{-1} \mathscr{R}_{(P)} \subset P \cdot\left(\mathscr{R}_{(P)}\right)$. This implies the relation $G_{(\bar{P})} / \bar{P} \cdot G_{(\bar{P})} \cong \mathscr{R}_{(P)} / P \cdot \mathscr{R}_{(P)}$ and the equality $N(P)=N(\bar{P})$.

Q.E.D.

(5.9.2) Let us denote the integer characterized in (5.9.1) by $N_{O_{X, x}}$ (or simply by $N_{x}$ or $N_{P}$ ) in this section. Lemma (5.9.1) says that $N_{P}$ depends only on the associated graded ring $G$.

(5.10) Z-graded regular rings. We will assume that $\operatorname{Spec}(G)-V\left(G_{+}\right)$is regular until (5.11). By (5.8.2), $\mathscr{R}_{(P)}$ is regular. We will show that the local ring $O_{X, x}=\left(\mathscr{R}_{(P)}\right)_{0}$ and the integer $N_{O_{X, x}}$ satisfy the conditions of (5.7).

For this purpose, we simplify the notation as follows:

(5.10.1) Let $R=\bigoplus_{n \in \mathbb{Z}} R_{n}$ be a regular graded ring which satisfies the following conditions;

(i) $R_{1} \neq 0$ and $R_{n}$ contains a unit of $R$ for some positive integer $n$ so that $R^{(n)}=R_{0}\left[t, t^{-1}\right]$ if $t \in R_{n}$ is a unit.

(ii) $R_{0}=B$ is a local ring with maximal ideal $m$.

Now, we define the integer $N_{B}$ by $N_{B}=\min \left\{n>0 \mid R_{n}\right.$ contains a unit of $\left.R\right\}$. Then we shall show the following: 
Claim (5.10.2)。 If we choose $t \in R_{N_{B}}$ so that $R_{N_{B}}=t \cdot B$, then $C=R /(t-1) \cdot R$ is a regular local ring.

Proof. Considering the homomorphism $\alpha: R \rightarrow C\left[U, U^{-1}\right]$, defined by $\alpha(g)=$ $(g \bmod (t-1) \cdot R) U^{m}$ for $g \in R_{m}$, where $U$ is an indeterminate, we see that $C\left[U, U^{-1}\right]$ is isomorphic to $R[U] /\left(U^{N_{B}}-t\right) \cdot R[U]$ as a graded ring. Obviously it suffices to prove that $C\left[U, U^{-1}\right]$ is regular. By the minimality of $N_{B}, R_{i} \cdot R_{j} \subset m \cdot R_{N_{B}}=m \cdot t$ for $0<$ $i, j<N_{B}$ with $i+j=N_{B}$. Hence our claim follows from the following lemma.

Lemma (5.10.3). Let $R=\bigoplus_{n \in \mathbb{Z}} R_{n}$ be a regular graded ring and $t \in \mathbb{R}_{N_{B}}$ be a unit of $R$. Assume $R$ satisfies the following conditions;

(i) $R_{0}=B$ is a local ring with maximal ideal $m$.

(ii) For every integers $i, j$ with $0<i, j<N_{B}$ and $i+j=N_{B}, R_{i} \cdot R_{j} \subset m \cdot t$.

Then $R^{*}=R[U] /\left(U^{N_{B}}-t\right) R[U]$ is regular, too, where $U$ is an indeterminate.

Proof. Let $n$ be the unique graded maximal ideal of $R$. Then $R / n=k\left[t, t^{-1}\right]$ and $R^{*} / n \cdot R^{*}=k\left[t, t^{-1}, U\right] /\left(U^{N_{B}}-t\right)=k\left[U, U^{-1}\right]$. Hence $n \cdot R^{*}$ is the maximal among the set of graded proper ideals of $R^{*}$. Since $R^{*}$ is flat over $R,\left(R^{*}\right)_{n \cdot R^{*}}$ is also flat over $\mathbb{R}_{n}$ by the base change theorem. Since $\left(R^{*}\right)_{n \cdot R^{*}} / n \cdot\left(R^{*}\right)_{n \cdot R^{*}}$ is regular, $\left(R^{*}\right)_{n \cdot R^{*}}$ is regular by (6.5.1) Chapter IV of [15]. Here $\left(R^{*}\right)_{Q}$ is regular for any proper graded prime ideal $Q$ of $R^{*}$. Therefore $R^{*}$ is a regular ring by Corollary 4.14 of [52].

(5.11) Now we will complete the proof of Theorem (5.6). Take a point $x$ of $X$ and put $B=O_{X, x}, R=O_{Y^{\prime}} \otimes_{O_{X}} B$. Then $R$ is regular by (5.8). Hence $B$ has only "cyclic quotient singularity" by (5.10) and (5.7). Moreover we show the following:

Claim (5.11.1)。 Let $P$ (resp. $\bar{P}$ ) be the homogeneous prime ideal of $\mathscr{R}$ (resp. G) associated to $x$, and $t$ be a homogeneous unit of $\mathscr{R}_{(P)}$ with the minimal positive degree as in (5.10).

Then $T^{-1}=u$ defines a member of a regular parameter system of $C=\mathscr{R}_{(P)} /(t-1) \mathscr{R}_{(P)}$.

Proof. Let $\bar{t}$ be the associated element of $G_{(p)}$. We obtain the exact sequence

$$
0 \longrightarrow\left(\frac{\mathscr{R}_{(P)}}{(t-1) \mathscr{R}_{(P)}}\right) \stackrel{T^{-1}}{\longrightarrow}\left(\frac{\mathscr{R}_{(P)}}{(t-1) \mathscr{R}_{(P)}}\right) \longrightarrow\left(\frac{G_{(P)}}{(\bar{t}-1) G_{(P)}}\right) \longrightarrow 0 .
$$

Here $G_{(P)}$ is regular and $\bar{t}$ gives a homogeneous unit of $G_{(P)}$ with the minimal positive degree by (5.9.1). Hence $\left(G_{(P)} /(\bar{t}-1) G_{(P)}\right)$ is a regular local ring by (5.10.1). Q.E.D.

Suppose $\operatorname{dim} A=2$. Then $X$ has only isolated singularities. So we can calculate a resolution of the singularity $(X, x)$ by a representative for the completion $B$. Here $u$ is a member of a regular parameter system of $C(5.11 .1)$, and $u \cdot C \cap B$ is the prime ideal defining $E$ in $X$. So, we have a "toric" resolution of $\operatorname{Spec}(B)$ where $E$ is transversal to the exceptional curve (cf. [7]).

This completes the proof of Theorem (5.6).

Example (5.12)。 (1) Let $(W, w)$ be a two-dimensional isolated singularity given as: $\left(O_{W, w}\right)^{\wedge}=A=k[[x, y, z]] /\left(x^{2}+y^{3}+z^{7}+y z^{5}\right)$ with the filtration $F$ on $A$ induced by putting the weights on each monomials as $\operatorname{wt}(x)=21, \operatorname{wt}(y)=14, \operatorname{wt}(z)=6$ (cf. (4.10)). 
Then $G \cong k[x, y, z] /\left(x^{2}+y^{3}+z^{7}\right)$. Then the orders of the cyclic groups, which produce the cyclic quotient singularities of $X$, depend only on $G$ by (5.9.1). For $P_{x}=(x)$, $P_{y}=(y)$ and $P_{z}=(z)$, we have the data $N_{P_{x}}=$ G.C.D. $(14,6)=2, N_{P_{y}}=$ G.C.D. $(21,6)=3$, and $N_{P_{z}}=G . C . D .(21,14)=7$. In fact the dual graph of the exceptional set of the minimal good resolution of $\operatorname{Spec}(A)$ is given as follows:

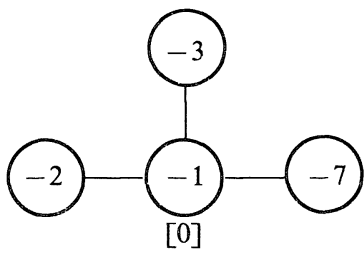

(2) Let $(W, w)$ be a three-dimensional isolated singularity given as: $\left(O_{W, w}\right)^{\wedge}=A=$ $k[[x, y, z, w]] /\left(x^{2}+y^{3}+z^{7}+w^{42}\right)$ with the filtration $F$ on $A$ induced by putting the weights on each monomials as $\operatorname{wt}(x)=21$, $\operatorname{wt}(y)=14$, $\operatorname{wt}(z)=6$, and $\operatorname{wt}(w)=1$ (cf. (4.10)). Then $G \cong k[x, y, z, w] /\left(x^{2}+y^{3}+z^{7}+w^{42}\right)$. As in (1), we will compute the orders of cyclic groups which produce the cyclic quotient singularities of $X$. For $P_{x}=(x), P_{y}=(y), P_{z}=(z)$, and $P_{w}=(w)$ of $G$, we have the data $N_{P_{x}}=$ G.C.D. $(14,6,1)=1$ $N_{P_{y}}=$ G.C.D. $(21,6,1)=1, N_{P_{z}}=$ G.C.D. $(21,14,1)=1$, and $N_{P_{w}}=$ G.C.D. $(21,14,6)=1$. These mean that $X$ is regular at the generic points on $V\left(P_{x}\right), V\left(P_{y}\right), V\left(P_{z}\right)$, and $V\left(P_{w}\right)$. For $P_{x, w}=(x, w), P_{y, w}=(y, w)$, and $P_{z, w}=(z, w)$ of $G$, we have $N_{P_{x, w}}=2, N_{P_{y, w}}=3$, and $N_{P_{z, w}}=7$. Here $X$ and $E=\operatorname{Proj}(G)$ have cyclic quotient singularities with same orders at each point (5.9.1).

Now, we will summarize our results obtained so far.

Summary (5.13). Let $(A, m)$ be a normal two-dimensional local ring. Suppose that there exists a filtration $\left\{F^{k}\right\}_{k \in \mathbb{Z}}$ on $A$ such that $G$ is an integral domain with isolated singularity. Then, in the minimal good resolution $h:\left(\tilde{X}, h^{-1}(w)\right) \rightarrow(W, w)$ with $W=\operatorname{Spec}(A)$ and $w=V(m)$, the dual graph of $h^{-1}(w)$ is star-shaped. Furthermore

(5.13.1) $h$ is obtained by the composition of morphisms as follows:

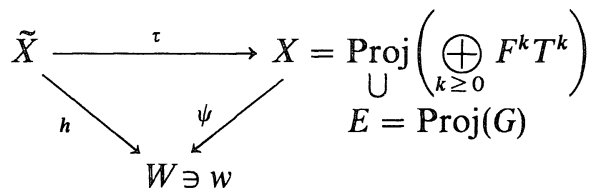

where $\psi$ is the filtered blowing-up of $W$ with respect to $\left\{F^{k}\right\}_{k \in \mathbf{Z}}$ and $\tau$ the morphism induced from the minimal resolution of singularities of $X$.

(5.13.2) The filtration $\left\{F^{k}\right\}_{k \in \mathbb{Z}}$ is reconstructed as $F^{k}=\psi_{*}\left(O_{X}(-k \cdot E)\right)$ for $k \in \mathbb{Z}$ in the above.

Remark (5.14). See [31] for other methods of local studies of singularities of the filtered blowing-ups of graded singularities of characteristic zero.

(5.15) Next, we shall study $X$ as a deformation of $\operatorname{Proj}\left(G^{\natural}\right)$. First, as is well known, $\operatorname{Spec}(A)$ is a small deformation of $\operatorname{Spec}(G)$ as follows: Let 


$$
\omega: Z^{\prime}=\operatorname{Spec}\left(\mathscr{R}^{\prime}\right) \rightarrow \operatorname{Spec}\left(k\left[T^{-1}\right]\right)=V
$$

be the natural map. Then $\omega^{-1}(0)=\operatorname{Spec}\left(\mathscr{R}^{\prime} / T^{-1} \mathscr{R}^{\prime}\right) \cong \operatorname{Spec}(G)$ and $\omega^{-1}(c)=$ $\operatorname{Spec}\left(\mathscr{R}^{\prime} /\left(T^{-1}-c\right) \mathscr{R}^{\prime}\right) \cong \operatorname{Spec}(A)$ for $c \in k$ and $c \neq 0$.

Moreover $X$ and $\operatorname{Proj}\left(G^{\natural}\right)$ are related as follows: Let $\left\{\left.\mathscr{R}\right|_{\ell} \mathscr{R}^{\prime}\right\}_{\ell \geq 0}$ be the filtration of $\mathscr{R}^{\prime}$ induced from $\left\{\left.\mathscr{R}\right|_{\ell}\right\}_{\ell \geq 0}$ on $\mathscr{R}$ (see (iii) of Remark (1.3)). Then we have the relations: $\left.\mathscr{R}\right|_{\ell} \mathscr{R}^{\prime}=\bigoplus_{s \in \mathbf{Z}}\left(\sum_{k \geq \ell} F^{k} \cdot F^{s^{-k}}\right) T^{s}=\bigoplus_{s \in \mathbb{Z}} F^{\max (s, \ell)} \cdot T^{s}$ for $\ell \geq 0$. Let $Y^{\prime \prime}=$ $\operatorname{Proj}\left(\left.\bigoplus_{\ell \geq 0} \mathscr{R}\right|_{\ell} \cdot \mathscr{R}^{\prime} \cdot U^{\ell}\right)$, where $U$ denotes an indeterminate. Then we obtain the following commutative diagram.

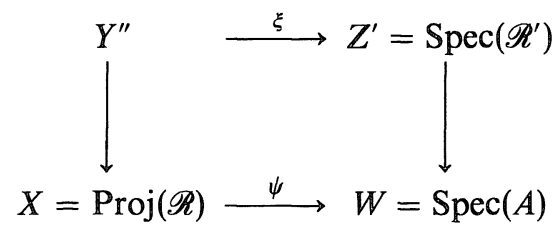

Claim (5.16).

$$
Y^{\prime \prime} \cong \operatorname{Spec}_{X}\left(\bigoplus_{k \in \mathbb{Z}} O_{X}(\max (k, 0)) T^{k}\right)=\operatorname{Spec}_{X}\left(\left(\left(\bigoplus_{k<-1} O_{X} \cdot T^{k}\right) \oplus\left(\bigoplus_{k \geq 0} O_{X}(k) \cdot T^{k}\right)\right)\right)
$$

Proof. Let $f \in F^{d}-F^{d+1}$. Then $f \cdot T^{d} \in \mathscr{R}_{d}$ and $\left.f \cdot T^{d} \cdot U^{d} \in \mathscr{R}\right|_{d} \cdot \mathscr{R}^{\prime}-\left.\mathscr{R}\right|_{d+1} \cdot \mathscr{R}^{\prime}$. In the neighborhood of the exceptional locus of $\xi$ in $Y^{\prime \prime}, Y^{\prime \prime}$ is covered by open sets of the form $\operatorname{Spec}\left(\left(\left(\left.\bigoplus_{\ell \geq 0} \mathscr{R}\right|_{\ell} \cdot \mathscr{R}^{\prime}\right)_{f \cdot T^{d} \cdot U^{d}}\right)_{0}\right)$. Here we have the relation:

$$
\begin{aligned}
\left(\left(\left.\bigoplus_{\ell \geq 0} \mathscr{R}\right|_{\ell} \cdot \mathscr{R}^{\prime}\right)_{f \cdot T^{d \cdot U^{d}}}\right)_{0} & =\bigcup_{s \geq 0}\left(\left.\mathscr{R}\right|_{d s} \cdot \mathscr{R}^{\prime}\right) /\left(f \cdot T^{d}\right)^{s} \\
& =\bigcup_{s \geq 0}\left(\sum_{k \in \mathbb{Z}}\left(\left(F^{\max (k, d s)}\right) / f^{s}\right) \cdot T^{k-d s}\right)=\sum_{k \in \mathbb{Z}} F^{\max (k, 0)}\left(A_{f}\right) \cdot T^{k} .
\end{aligned}
$$

Q.E.D.

Proposition (5.17). Let the situation be as above. Then the morphism $\xi$ is defined over the scheme $\operatorname{Spec}\left(k\left[T^{-1}\right]\right)$ as follows:

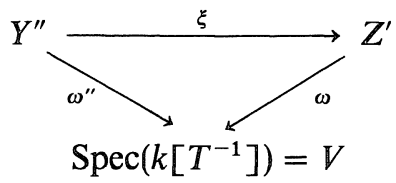

On the point $0=V\left(T^{-1}\right)$ of $V$, $\xi$ is the filtered blowing-up $\omega^{\prime \prime-1}(0)=\operatorname{Proj}\left(G^{\natural}\right) \rightarrow$ $\omega^{-1}(0)=\operatorname{Spec}(G)$ induced by the grading of $G$.

On the point $c=V\left(T^{-1}-c\right)$ of $V$ with $0 \neq c \in k, \xi$ is the filtered blowing-up $\omega^{\prime \prime-1}(c) \cong X \rightarrow \omega^{-1}(c) \cong \operatorname{Spec}(A)$ induced by the filtration $\left\{F^{k}\right\}_{k \in \mathbf{Z}}$. 
Proof. We have the relations:

$$
\begin{aligned}
\omega^{\prime \prime-1}(0) & =\operatorname{Spec}_{X}\left(\bigoplus_{k \in \mathbb{Z}}\left(O_{X}(\max (k, 0)) / O_{X}(\max (k+1,0)) T^{k}\right)\right. \\
& =\operatorname{Spec}_{X}\left(\bigoplus_{k \geq 0}\left(O_{X}(k) / O_{X}(k+1)\right) T^{k}\right) \\
& =\operatorname{Spec}_{X}\left(\bigoplus_{k \geq 0} O_{E}(k)\right)=\operatorname{Proj}\left(G^{\natural}\right), \quad \text { and } \\
\omega^{\prime \prime-1}(c) & =\operatorname{Spec}_{X}\left(\bigoplus_{k \in \mathbb{Z}}\left(O_{X}(\max (k, 0)) /\left(T^{-1}-c\right) O_{X}(\max (k+1,0))\right) T^{k}\right) \\
& =\operatorname{Spec}_{X}\left(O_{X}\right) .
\end{aligned}
$$

The remaining assertions are easy.

Corollary (5.18). Let the situation be as in Proposition (5.17) and the conditions of Lemma (5.2) hold. Then the singularities of $X$ are obtained as small deformation of singularities of $\operatorname{Proj}\left(R(E, D)^{\natural}\right)$.

\section{Chapter 2. Normal Two-Dimensional Singularities with "Star-Shaped" Resolution}

\section{§ 6. "Star-Shaped" Resolution and the Filtration}

(6.1) Throughout this chapter, we assume $(A, m)$ is a normal two-dimensional local ring over an algebraically closed field $k$. By Theorem (5.6), if $A$ is a local ring which possesses a filtration $\left\{F^{k}\right\}_{k \in \mathbf{Z}}$ such that $G$ is an integral domain with an isolated singularity, then the dual graph of exceptional set in the minimal good resolution of the singularity is star-shaped written as follows:

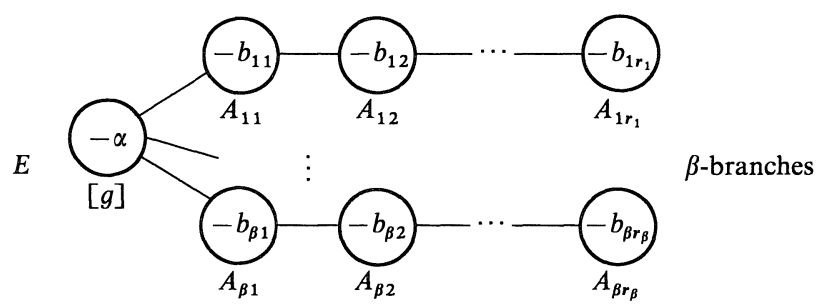

Here we shall employ the standard notation as in [41] and [43].

In this chapter, conversely, we shall study the filtrations on $A$ from the resolution as above.

(6.2) Let $f:\left(\tilde{X}, f^{-1}(w)\right) \rightarrow(W, w)$ be a resolution of singularity $(W, w)$ such that the dual graph of $f^{-1}(w)$ is a star-shaped graph as above. We contract the branches $\bigcup_{i, j} A_{i j}$ to normal points. Then we get the normal surface $X$ and the commutative diagram as follows: 


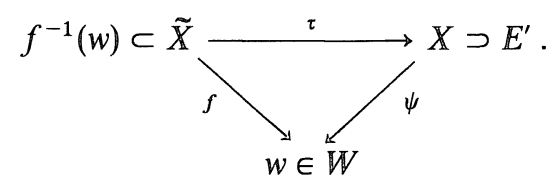

Here $E^{\prime}$ is the image of $E$. $\tau$ and $\psi$ are the morphisms induced canonically.

By Pinkham [34] and Demazure [7], we can construct the normal two-dimensional graded ring $R(E, D)$ as follows:

Let $Q$ be an integral divisor on $E$ with relation $N_{E \mid \tilde{X}}^{-1}=O_{E}(Q)$, and $P_{i}$ the intersection point $E \cap A_{i 1}$ for $i=1, \ldots, \beta$. Let the rational divisor $D$ on $E$ be as follows: $D=Q-\sum_{i=1}^{\beta} q_{i} P_{i}$, where the rational number $q_{i}$ is defined by continued fraction

$$
\frac{1}{q_{i}}=\left[b_{i 1}, b_{i 2}, \ldots, b_{i r_{1}}\right]=b_{i 1}-\frac{1}{b_{i 2}-\frac{1}{\cdots-\frac{1}{b_{i r_{i}}}}}, \quad \text { for } \quad i=1, \ldots, \beta
$$

Let $R(E, D)=\bigoplus_{k \geq 0} H^{0}\left(E, O_{E}(k D)\right) T^{k} \subset k(E)[T]$, where $k(E)$ is the field of rational functions of $E$, and $T$ an indeterminate.

Then the dual graph of the minimal good resolution of $\operatorname{Spec}(R(E, D))$ at $R_{+}$is the same as that of $(W, w)$. We shall relate $R(E, D)$ with $A$ in the following.

Theorem (6.3). Let $\tau:\left(\tilde{X}, f^{-1}(w)\right) \rightarrow\left(X, E^{\prime}\right)$ and $\psi:\left(X, E^{\prime}\right) \rightarrow(W, w)$ be as above. Let the filtration $\left\{F^{k}\right\}_{k \in \mathbb{Z}}$ on $A$ be defined by $F^{k}=\psi_{*}\left(O_{X}\left(-k \cdot E^{\prime}\right)\right)$ for $k \in \mathbb{Z}$.

Then $G=\bigoplus_{k \geq 0}\left(F^{k} / F^{k+1}\right) T^{k}$ is an integral domain with isolated singularities. Further

(6.3.1) The Pinkham-Demazure construction $R(E, D)$ is the normalization of $G$. We have the exact sequence of graded G-modules

$$
0 \rightarrow G \rightarrow \mathbb{R}(E, D) \rightarrow U \cong H_{G_{+}}^{1}(G) \rightarrow 0
$$

where

$$
U=\bigoplus_{k \geq 0} \mathbb{K e r}\left\{R^{1} \psi_{*}\left(O_{X}\left(-(k+1) \cdot E^{\prime}\right)\right) \rightarrow \mathbb{R}^{1} \psi_{*}\left(O_{X}\left(-k \cdot E^{\prime}\right)\right)\right\} T^{k}
$$

We can identify $E$ and $E^{\prime}$ by $\tau$.

(6.3.2) We obtain the equality (cf. Remark (6.20))

$$
\begin{aligned}
1_{A}(U) & =\sum_{k \geq 0} \operatorname{dim} H^{1}\left(E, O_{E}(k D)\right)-\operatorname{dim} R^{1} \psi_{*}\left(O_{X}\right) \\
& =p_{g}(R(E, D))-p_{g}(A)
\end{aligned}
$$

Furthermore the following conditions are equivalent to each other:

(i) $G$ is normal.

(ii) $R^{1} \psi_{*}\left(O_{X}(-(k+1) \cdot E)\right) \rightarrow R^{1} \psi_{*}\left(O_{X}(-k \cdot E)\right)$ is injective for $k \in \mathbb{Z}$.

(ii) $\quad R^{1} \psi_{*}\left(O_{X}(-(k+1) \cdot E)\right) \rightarrow H_{m}^{2}(A) \cong H^{1}\left(X-E, O_{X}(-k \cdot E)\right)$ is injective for $k \in \mathbb{Z}$.

(iii) $\quad p_{g}(R(E, D))=p_{g}(A)$. 
Proof. The key lemma is the following:

Lemma (6.4). Let the situation be as in (6.2). Then we have the following isomorphisms:

$$
O_{X}\left(-k \cdot E^{\prime}\right) / O_{X}\left(-(k+1) \cdot E^{\prime}\right) \cong \tau_{*}\left(O_{E}(k D)\right) \quad \text { on } X \quad \text { for } \quad k \in \mathbb{Z}
$$

By this lemma, we have the condition (ii) of Lemma (5.2). The assertions of Theorem (6.3) follow from Proposition (1.17), Lemma (5.2), and Remark (5.5). Since $G$ is an integral domain, our $\left\{F^{k}\right\}_{k \in \mathbb{Z}}$ is determined as in (2.2) (cf. (5.13)).

Before we proceed to the proof of Lemma (6.4), we shall give some easy consequences of Theorem (6.3) here.

First note the following fact:

(6.5) Since $\psi_{*}\left(O_{X}\right) \rightarrow \psi_{*}\left(O_{E}\right)=k$ is surjective, $R^{1} \psi_{*}\left(O_{X}(-E)\right) \rightarrow R^{1} \psi_{*}\left(O_{X}\right)$ is always injective.

Corollary (6.6). Let the situation be as in (6.2). If $a(R(E, D)) \leq 1$, i.e., $H^{1}\left(E, O_{E}(k D)\right)=0$ for $k \geq 2$, then $G=R(E, D)$. In this case, $A$ is a Gorenstein (resp. a complete intersection, resp. a hypersurface) when $R(E, D)$ is Gorenstein (resp. a complete intersection, resp. a hypersurface).

Proof. By our assumption and Lemma (6.4), we obtain $R^{1} \psi_{*}\left(O_{X}(-k E)\right)=0$ for $k \geq 2$ (cf. Proposition (1.13) and (5.5)). Hence $U=0$ by (6.5).

Remark (6.7). Let $\varepsilon(R(E, D))=\min \left\{k \in \mathbb{Z} \mid k>0, R(E, D)_{k} \neq 0\right\}$. Then the condition $a(R(E, D)) \leq \varepsilon(R(E, D))$ implies $U=0$ by similar argument.

Example (6.8). Let $(W, w)$ be a singularity with minimal good resolution $f:\left(\tilde{X}, f^{-1}(w)\right) \rightarrow(W, w)$. Suppose the dual graph $\Gamma$ of $f^{-1}(w)$ is a star-shaped graph given as follows:

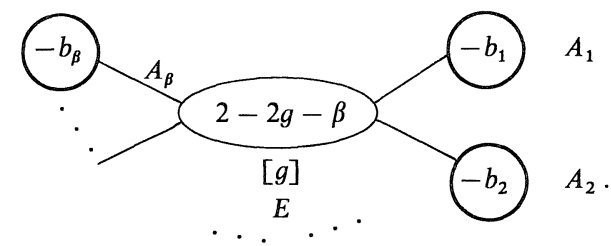

The normal class of $E$ is $-K_{E}-P_{1}-P_{2}-\cdots-P_{\beta}$, where $\beta \geq 0($ resp. $\beta>0$, resp. $\left.\beta-2>\sum_{i=1}^{\beta} \frac{1}{b_{i}}\right)$ if $g \geq 2$ (resp. $g=1$, resp. $g=0$ ).

Then $R(E, D)$, with $D=K_{E}+\sum_{i=1}^{\beta} \frac{b_{i}-1}{b_{i}} P_{i}, \quad$ is a Gorenstein domain with $a(R(E, D))=1((2.9)$ of $[45])$. Hence $G=R(E, D)$ by Corollary (6.6). Therefore $(W, w)$ is always a Gorenstein domain. 
In case $a(R(E, D)) \geq 2$, our $U$ is not necessarily zero as in the following example which has been discussed in [37] (For the relation between the explicit form in [37] and the description below, we refer to [21]).

Example (6.9) (O. Riemenschneider [37]). Let $E$ be a non-singular algebraic curve of genus $g$ and $L \rightarrow E$ a line bundle of positive degree. We denote the invertible sheaf associated to $L$ also by the same notation $L$ in the below. Let $\xi \in H^{1}(E, L) \cong$ $\operatorname{Ext}_{E}^{1}\left(L^{-1}, O_{E}\right)$. Let $M$ be the locally free $O_{E}$-module of rank 2 obtained from the following extension of $L^{-1}$ by $O_{E}$ associated to the cohomology class $\xi$

$$
0 \rightarrow O_{E} \rightarrow M \rightarrow L^{-1} \rightarrow 0 \text {. }
$$

Let $S$ be the ruled surface over $E$ obtained as $S=\operatorname{Proj}\left(\bigoplus_{d \geq 0} S^{d}(M)\right) \rightarrow E$ and $X$ a suitable neighborhood of the negative section, which we also denote by $E$, in $S$ such that $((X, E)$ blows down to a normal singularity $(W, w)$. We are interested in $U$ for $(W, w)$. In our terminology, Riemenschneider's arguments say " $H^{0}\left(\mathbb{E}, L^{k}\right) \cdot \xi=0$ in $H^{1}\left(E, L^{k+1}\right)$ for $k \geq 1$, if $U=0$ ". Further he shows the existence of $L$ with property $H^{0}(E, L) H^{1}(E, L) \neq 0$ when $g \geq 2$. In particular, we can construct examples with $U \neq 0$ when $g \geq 2$.

We have assumed that we always take the representative $A$ of local ring $O_{W, w}$ as being essentially of finite type over a field. For this reason, we shall replace the local ring up to analytic equivalence (see the studies of [19], [20]).

(6.10) The proof of Lemma (6.4) is divided into several steps. The key step to the proof is to prove the following equalities

$$
\tau^{-1}\left(O_{X}\left(-k E^{\prime}\right)\right) \otimes_{o_{\tilde{X}}} O_{E}=O_{E}(k D) \quad \text { on } E \quad \text { for any } \quad k \in \mathbb{Z} .
$$

In order to study the inverse image of divisorial sheaves on $X$ by $\tau$, we shall use Giraud's terminologies [10] (Similar description is introduced also in [27].). Here the inverse image $\tau^{-1}(\mathscr{M})$ of a coherent $O_{X^{-}}$sheaf $\mathscr{M}$ is defined by $\tau^{-1}(\mathscr{M})=$ $\left(\tau^{*}(\mathscr{M}) \otimes_{O_{X}} O_{\tilde{X}}\right) / O_{\tilde{X}}$-torsion.

(6.11) Giraud's inverse image [10]. Let $X$ be a normal two-dimensional scheme which can be embedded in an algebraic variety, and $\tau: \widetilde{X} \rightarrow X$ a proper modification (i.e., a proper birational morphism) such that $\tilde{X}$ is non-singular. The exceptional locus $B$ of $\tau$ in $\tilde{X}$ is the set of points where $\tau$ is not isomorphic. Let $B=\bigcup_{i=1}^{r} A_{i}$ be the decomposition into irreducible components. Let us denote the divisors on $\tilde{X}$ whose supports are contained in $B$ as follows: $N S(\tau, \mathbb{Z})=\bigoplus_{i=1}^{r} \mathbb{Z} A_{i}$, and $N S(\tau, \mathbb{Q})=\bigoplus_{i=1}^{r} \mathbb{Q} A_{i}$. For $D \in N S(\tau, \mathbb{Z})$, we define the intersection number $D \cdot A_{i}$ by $D \cdot A_{i}=$ degree of $O_{A_{i}} \otimes$ $O_{\tilde{X}}(D)$, and extend it on $N S(\tau, \mathbb{Q})$ by $\otimes_{\mathbb{Z}} \mathbb{Q}$.

Definition (6.11.1). For $D \in N S(\tau, \mathbb{Q})$, we denote $D \gg 0$ if $D A_{i} \leq 0$ holds for any $i$.

In particular, $D$ is effective (we shall denote as $D \geq 0$ ) when $D \geq 0$. 
Lemma-Definition (6.11.2). For any $D \in N S(\tau, \mathbb{Q})$, there exists a unique divisor $[D]_{G} \in N S(\tau, \mathbb{Z})$ such that

(1) $D \leq<[D]_{G}$,

(2) if $W \in N S(\tau, \mathbb{Z})$ and if $D \leq W$, then $[D]_{G} \leq W$.

In fact, we can easily prove the relation $[D]_{G}=\min \{W \in N S(\tau, \mathbb{Z}) \mid D \leq<W\}$.

Definition (6.11.3). For an invertible sheaf $\mathscr{L}$ on $\tilde{X}, e_{\tau}(\mathscr{L})$ is the unique element in $N S(\tau, \mathbf{Q})$ which satisfies $e_{\tau}(\mathscr{L}) A_{i}=\operatorname{deg}\left(\mathscr{L} \otimes O_{A_{\imath}}\right)$ for all $i$.

Proposition (6.11.4). Let $\tau: \tilde{X} \rightarrow X$ be as above. Let $\mathscr{M}$ be a divisorial sheaf on $X$ and denote as $\mathscr{M}=O_{X}(M)$ by using a Weil divisor $M$ on $X$. Then there exists a pair $(\mathscr{L}, u)$ where $\mathscr{L}$ is an invertible sheaf on $\tilde{X}$ such that $\left[e_{\tau}(\mathscr{L})\right]_{G}=0$ and $u:\left.\tau_{*}(\mathscr{L})\right|_{X-\tau(B)} \rightarrow$ $\left.\mathscr{M}\right|_{X-\tau(B)}$ is an isomorphism. The pair $(\mathscr{L}, u)$ is unique up to a unique isomorphism. Furthermore $\mathscr{M}=\tau_{*}(\mathscr{L})$.

We shall call this $\mathscr{L}$ Giraud's inverse image of $\mathscr{M}$ by $\tau$. Let $M_{1}$ be the strict transform of $M$ by $\tau$. Then we can easily prove the relation:

$$
\mathscr{L}=O_{\tilde{X}}(L), \quad \text { where } \quad L=M_{1}-\left[e_{\tau}\left(M_{1}\right)\right]_{G} .
$$

(We shall call this $L$ Giraud's inverse image of the Weil divisor $M$ by $\tau$.)

Proposition (6.11.5). Let the situation be as above. If $R^{1} \tau_{*}\left(O_{\tilde{X}}\right)=0$, then $\mathscr{L}=$ $\tau^{-1}(\mathscr{M})$ in the sense of $(6.10)$ and $R^{1} \tau_{*}(\mathscr{L})=0$.

(6.12) A computation sequence for Giraud's inverse image ((1.4) of [10]). Let $Z$ be an integral divisor on $\tilde{X}$ such that $Z$ equals $M_{1}$ outside of $B$ and satisfies the relation $Z \geq L=M_{1}-\left[e_{\tau}\left(M_{1}\right)\right]_{G}$ (cf. (6.12.2) below for existence of such $Z$ ). Then we can find out $L$ explicitly from $Z$ as follows:

(6.12.1) Let us define a sequence of integral divisors $\left\{Z^{(k)}\right\}_{k \geq 0}$ inductively as follows: $Z^{(0)}=Z, Z^{(i)}=Z^{(i-1)}-A_{j_{i}}$ when there exists $A_{j_{i}}$ with $Z^{(i-1)} A_{j_{\imath}}<0$.

Then this procedure ends in finite steps. Let $Z^{(s)}$ be the last member in the above. Then $Z^{(s)}=L$.

Proof. By definition, $L A_{i} \geq 0$ for any component $A_{i}$ of $B$. Hence $(Z-L) A_{i}<0$ holds for $A_{i}$ with $Z A_{i}<0$. Since $Z-L$ is effective, $Z-L \geq A_{i}$ holds for such $A_{i}$. Hence $L \leq Z-A_{j_{1}}=Z^{(1)}$. Similarly we obtain the relations $L \leq Z^{(k)}$ for all $k$. Therefore the sequence (6.12.1) ends in finite steps. Let $Z^{(s)}$ be the last of this sequence. By definition, the relation $e_{\tau}\left(Z^{(s)}\right) \leq<0$ holds. Hence $\left[e_{\tau}\left(Z^{(s)}\right)\right]_{G} \leq 0$. On the other hand, $0 \leq Z^{(s)}-L=\left[Z^{(s)}-L\right]_{G}=\left[Z^{(s)}-L+e_{\tau}(L)\right]_{G}=\left[e_{\tau}(L)\right]_{G}$.

(6.12.2) Actually, if we set $Z=M_{1}-\left\{e_{\tau}\left(M_{1}\right)\right\}$, then we can choose a computation sequence for $Z$ as in (6.12.1). Here $\{\alpha\}$ means $\min \{x \in \mathbb{Z} \mid \alpha \leq x\}$ for a rational number $\alpha$ and $\{D\}$ means $\sum_{i=1}^{r}\left\{\alpha_{i}\right\} A_{i}$ for a $Q$-divisor $D=\sum_{i=1}^{r} \alpha_{i} A_{i}$. The divisors of the form $M_{1}-\left\{e_{\tau}\left(M_{1}\right)\right\}$ have been studied precisely by D. Mumford and F. Sakai (see [38]).

(6.13) Here we shall assume that the exceptional locus $B$ of $\tau$ is of the form: 


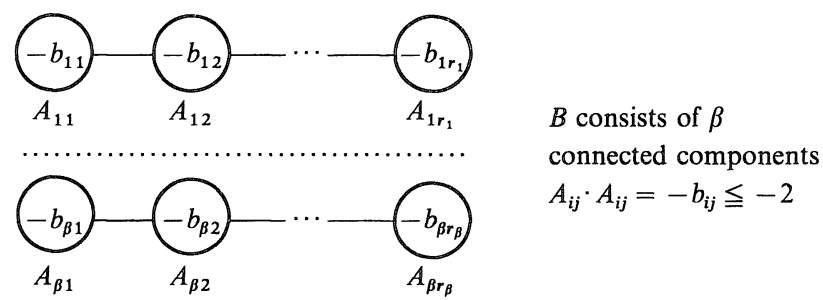

Lemma (6.14). Let the situation be as above. Then the coefficients of the divisors $Z=M_{1}-\left\{e_{\tau}\left(M_{1}\right)\right\}$ and $L=M_{1}-\left[e_{\tau}\left(M_{1}\right)\right]_{G}$ are equal on the irreducible components $A_{i 1}$ and $A_{\text {ir }}$ for $i=1, \ldots, \beta$.

Now, we will prove (6.10.1) assuming (6.14).

(6.15) Proof of the relations (6.10.1). By Proposition (6.11.1) and Lemma (6.14), we obtain the relations

$$
\begin{aligned}
\tau^{-1}\left(O_{X}\left(-k \cdot E^{\prime}\right)\right) \otimes_{O_{\tilde{X}}} O_{E} & =O_{\tilde{X}}\left(-k \cdot E-\left[e_{\tau}(-k \cdot E)\right]_{G}\right) \otimes_{O_{\tilde{X}}} O_{E} \\
& =O_{\tilde{X}}\left(-k \cdot E-\left\{e_{\tau}(-k \cdot E)\right\}\right) \otimes_{O_{\tilde{X}}} O_{E}
\end{aligned}
$$

on $E$ for any $k \in \mathbb{Z}$. Further one can easily show the relation

$$
e_{\tau}(E)=-\sum_{i=1}^{\beta}\left(\sum_{j=1}^{r_{i}} \frac{1}{\prod_{m=1}^{j}\left[b_{i m}, b_{i m+1}, \ldots, b_{i r_{i}}\right]} A_{i j}\right) .
$$

(6.16) Proof of Lemma (6.14). The assertion of Lemma (6.14) is local on X. Hence we assume the exceptional locus $B$ is connected as follows:

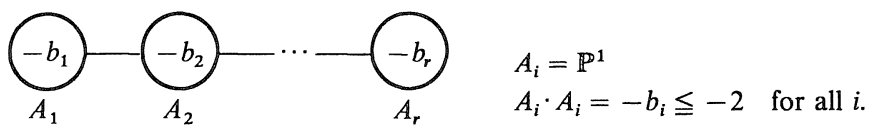

Let us denote $e_{\tau}\left(M_{1}\right)$ and $e_{\tau}(Z)$ in the form:

$$
e_{\tau}\left(M_{1}\right)=\sum_{i=1}^{r} y_{i} A_{i} \quad \text { with } \quad y_{i} \in \mathbb{Q} \quad \text { for } \quad i=1, \ldots, r,
$$

and

$$
e_{\tau}(Z)=\sum_{i=1}^{r} \alpha_{i} A_{i} \quad \text { with } \quad \alpha_{i} \in \mathbb{Q} \quad \text { for } \quad i=1, \ldots, r
$$

Then, by definition, we obtain the relations

$$
\alpha_{i}=y_{i}-\left\{y_{i}\right\} \quad \text { for } i=1, \ldots, r .
$$

We have the relations

$$
\begin{aligned}
& Z A_{1}=e_{\tau}(Z) A_{1}=-b_{1} \alpha_{1}+\alpha_{2}, \\
& Z A_{i}=e_{\tau}(Z) A_{i}=-b_{i} \alpha_{i}+\alpha_{i-1}+\alpha_{i+1} \quad \text { for } 1<i<r, \\
& Z A_{r}=e_{\tau}(Z) A_{r}=-b_{r} \alpha_{r}+\alpha_{r-1} .
\end{aligned}
$$


We have to show $A_{1}$ and $A_{r}$ do not appear as $A_{j_{l}}$ in a computation sequence (6.11.1) $\left\{Z^{(k)} \mid k=0, \ldots, s, Z^{(0)}=Z\right.$ and $\left.Z^{(s)}=L\right\}$. Or, equivalently, $Z^{(k)} A_{1}$ and $Z^{(k)} A_{r}$ are non-negative for $k=0,1, \ldots, s$. Since the situation is symmetric with $A_{1}$ and $A_{r}$, we will prove the relation $Z^{(k)} A_{1} \geq 0$ for $0 \leq k \leq s$.

Now, take any integers $i, j$ with $1 \leq i \leq j \leq r$. Then we have

$$
\begin{aligned}
Z\left(A_{i}+\cdots+A_{j}\right) & =\alpha_{i-1}+\alpha_{i+1}+\left(1-b_{i}\right) \alpha_{i}+\left(1-b_{j}\right) \alpha_{j}+\sum_{m=i+1}^{j-1}\left(2-b_{m}\right) \alpha_{m} \\
& \geq \alpha_{i-1}+\alpha_{j+1}
\end{aligned}
$$

(if $i=1$ or $j=r$, put $\alpha_{0}=0$ and $\alpha_{r+1}=0$ ).

Hence we have the inequalities

$$
\begin{aligned}
& Z\left(A_{i}+\cdots+A_{j}\right) \geq-1, \\
& Z\left(A_{1}+\cdots+A_{j}\right) \geq 0,
\end{aligned}
$$

for every $i$, with $1 \leq i \leq j \leq r$. From these inequalities, we can easily see;

Claim (6.16.2). (1) $Z A_{i} \geq-1$ for every $i$ and $Z A_{1} \geq 0$.

(2) If $Z A_{i}=-1$ for some $i$, then there exists an integer $m, 1 \leq m<i$ with $Z A_{m} \geq 1$.

(3) If $Z A_{i}=Z A_{j}=-1$ for some integers $i$ and $j$ with $i<j$, then there exists an integer $m, i<m<j$ with $Z A_{m} \geq 1$.

Now, assume that $Z A_{i}=-1$ for some $i$, and put $Z^{(1)}=Z-A_{i}$, then we have the relations $Z^{(1)} A_{i}=-1+b_{i} \geq 1, Z^{(1)} A_{i-1}=Z A_{i-1}-1$, and $Z^{(1)} A_{i+1}=Z A_{i+1}-1$.

Thus the conditions of (6.12.2) hold, if we put $Z^{(1)}$ in place of $Z$. Hence we can prove the conditions of (6.16.2) for every $Z^{(k)}, k=0, \ldots, s$ in place of $Z$ inductively.

This completes the proof of Lemma (6.14).

Using the relation (6.10.1), we can prove Lemma (6.4).

(6.17) Proof of Lemma (6.4). Let $L_{-k}$ be Giraud's inverse image (6.11) of the Weil divisor $-k E^{\prime}$ by $\tau$. Since $\tau_{*}\left(O_{\tilde{X}}\left(L_{-k}\right)\right)$ is reflexive and $L_{-k-1} \leq L_{-k}-E$, the sheaf $\tau_{*}\left(O_{\tilde{X}}\left(L_{-k}-E\right)\right)$ is also reflexive and equal to $O_{X}\left(-(k+1) E^{\prime}\right)$. Therefore we obtain the following exact sequence on $X$ :

$$
\begin{aligned}
& 0 \rightarrow O_{X}\left(-(k+1) E^{\prime}\right) \rightarrow O_{X}\left(-k E^{\prime}\right) \rightarrow \tau_{*}\left(O_{E}(k D)\right) \rightarrow R^{1} \tau_{*}\left(O_{\tilde{X}}\left(L_{-k}-E\right)\right) \rightarrow R^{1} \tau_{*}\left(O_{\tilde{X}}\left(L_{-k}\right)\right) \\
& \tau_{*}\left(O_{\tilde{X}}\left(L_{-k}-E\right)\right) \quad \tau_{*}\left(O_{\tilde{X}}\left(L_{-k}\right)\right)
\end{aligned}
$$

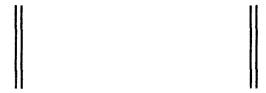

where $R^{1} \tau_{*}\left(O_{\tilde{X}}\left(L_{-k}\right)\right)=0$ by $(6.11 .5)$.

Hence, what we have to show is the vanishing of $R^{1} \tau_{*}\left(O_{\tilde{X}}\left(L_{-k}-E\right)\right)$.

Sublemma (6.17.1). Let effective divisor $W_{-k} \in N S(\tau, \mathbb{Z})$ be as follows: $W_{-k}=$ $L_{-k-1}-L_{-k}+E$ for $k \in \mathbb{Z}$. Then we have the equality $\sum_{q \in X} \operatorname{dim}\left(R^{1} \tau_{*}\left(O_{\tilde{X}}\left(L_{-k}-E\right)\right)\right)_{q}=$ 
$p_{a}\left(W_{-k}\right)-1+\left(W_{-k}\right) E-\left(W_{-k}\right)\left(L_{-k}\right)$, where $p_{a}\left(W_{-k}\right)$ is the virtual arithmetic genus of $W_{-k}$ defined as follows: $p_{a}\left(W_{-k}\right)=1-\chi\left(O_{W_{-k}}\right)$.

Proof of Sublemma (6.17.1). Let $\bar{X}$ be a normal projective variety which contains $X$ as a Zariski open set and is non-singular at points of $\bar{X}-X$. Let $\bar{\tau}: \bar{X}^{\sim} \rightarrow \bar{X}$ be the morphism induced canonically from $\tau: \tilde{X} \rightarrow X$. Since $\bar{\tau}_{*}\left(O_{\bar{X} \sim}\left(L_{-k-1}\right)\right)=$ $\bar{\tau}_{*}\left(O_{\bar{X}^{\sim}}\left(L_{-k}-E\right)\right)=O_{\bar{X}}\left(-(k+1) E^{\prime}\right) \quad$ and $\quad R^{1} \tau_{*}\left(O_{\bar{X} \sim}\left(L_{-k-1}\right)\right)=0$, we obtain the relations $\bar{\tau}_{*}\left(O_{\bar{X}^{\sim}}\left(L_{-k}-E\right) / O_{\bar{X}^{\sim}}\left(L_{-k-1}\right)\right)=0 \quad$ and $\quad R^{1} \bar{\tau}_{*}\left(O_{\bar{X} \sim}\left(L_{-k}-E\right) / O_{\bar{X} \sim}\left(L_{-k-1}\right)\right)=$ $R^{1} \bar{\tau}_{*}\left(O_{\bar{X} \sim}\left(L_{-k}-E\right)\right)$. By the Riemann-Roch formula for surfaces, we obtain the following equalities:

$$
\begin{aligned}
& \sum_{q \in X} \operatorname{dim}\left(R^{1} \bar{\tau}_{*}\left(O_{\bar{X}^{\sim}}\left(L_{-k}-E\right)\right)\right)_{q}=-\chi\left(\bar{X}^{\sim}, O_{\bar{X}^{\sim}}\left(L_{-k}-E\right) / O_{\bar{X}^{\sim}}\left(L_{-k-1}\right)\right) \\
&=\chi\left(\bar{X}^{\sim}, O_{\bar{X}^{\sim}}\left(L_{-k-1}\right)\right)-\chi\left(\bar{X}^{\sim}, O_{\bar{X}^{\sim}}\left(L_{-k}-E\right)\right) \\
&=\frac{1}{2}\left\{-K_{\bar{X}^{\sim}} L_{-k-1}+\left(L_{-k-1}\right)^{2}\right\}+\chi\left(\bar{X}^{\sim}, O_{\bar{X}^{\sim}}\right) \\
& \quad-\frac{1}{2}\left\{-K_{\bar{X}^{\sim}}\left(L_{-k}-E\right)+\left(L_{-k}-E\right)^{2}\right\}-\chi\left(\bar{X}^{\sim}, O_{\bar{X}^{\sim}}\right) \\
&=\frac{1}{2}\left\{-K_{\bar{X}^{\sim}}\left(L_{-k}-E\right)+K_{\bar{X}^{\sim}} W_{-k}+\left(L_{-k}-E\right)^{2}\right. \\
&\left.-2\left(L_{-k}-E\right) W_{-k}+\left(W_{-k}\right)^{2}\right\}-\frac{1}{2}\left\{-K_{\bar{X}^{\sim}}\left(L_{-k}-E\right)+\left(L_{-k}-E\right)^{2}\right\} \\
&= p_{a}\left(W_{-k}\right)-1-W_{-k}\left(L_{-k}-E\right)
\end{aligned}
$$

where $K_{\bar{X}^{\sim}}$ is the canonical divisor of $\bar{X}^{\sim}$.

Q.E.D. for Sublemma (6.17.1).

(6.17.2) We shall complete the proof of Lemma (6.4). Let $W_{i,-k}$ be the part of $W_{-k}$ over connected component $\bigcup_{j=1}^{r_{i}} A_{i j}$. Then $W_{-k}=W_{1,-k}+W_{2,-k}+\cdots+W_{\beta,-k}$. We obtain

$$
p_{a}\left(W_{-k}\right)-1-W_{-k}\left(L_{-k}-E\right)=\sum_{i=1}^{\beta}\left\{p_{a}\left(W_{i,-k}\right)-1-W_{i,-k}\left(L_{-k}-E\right)\right\} .
$$

We shall show the relation $p_{a}\left(W_{i,-k}\right)-1-W_{i,-k}\left(L_{-k}-E\right) \leq 0$ for each $i$. In case $W_{i,-k}=0$, this is obvious. Suppose $W_{i,-k}$ is a non-zero effective divisor. First, $p_{a}\left(W_{i,-k}\right) \leq 0$ follows from the fact $R^{1} \tau_{*}\left(O_{\bar{X}^{\sim}}\right)=0$ ([1], [2]). Second, $W_{i,-k} \cdot L_{-k} \geq 0$ follows from the fact $\left[e_{\tau}\left(L_{-k}\right)\right]_{G}=0$ (see (6.11)). Third, by (6.10.1);

the coefficient of $W_{i,-k}$ at the irreducible component $A_{i 1}$

$$
=-\left\{\frac{k}{\left[b_{i 1}, \ldots, b_{i r_{1}}\right]}\right\}+\left\{\frac{k+1}{\left[b_{i 1}, \ldots, b_{i r_{l}}\right]}\right\}<1+\frac{1}{\left[b_{i 1}, \ldots, b_{i r_{l}}\right]}<2 .
$$

Hence $E W_{i,-k} \leq 1$, and $p_{a}\left(W_{i,-k}\right)-1+E W_{i,-k}-W_{i,-k} L_{-k} \leq 0$.

Q.E.D. for Lemma (6.4). 
Example (6.18) (Computation sequence for Giraud's inverse image). Let the situation be as in Theorem (6.3) and the dual graph of $f^{-1}(w)$ be as follows:

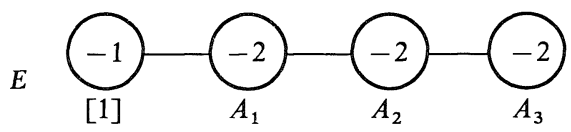

Here $A_{1} \cup A_{2} \cup A_{3}$ is contracted to a normal point by $\tau: \tilde{X} \rightarrow X$. Let the divisor $L_{-k}$ on $\tilde{X}$ be Giraud's inverse image of Weil divisor $-k E$ on $X$, for $k \in \mathbb{Z}$. If we denote $-k E-\left\{e_{\tau}(-k E)\right\}$ by $Z_{-k}$ on $\tilde{X}$, we have

$$
Z_{-k}=-k E-\left\{\frac{3}{4} k\right\} A_{1}-\left\{\frac{2}{4} k\right\} A_{2}-\left\{\frac{1}{4} k\right\} A_{3} \quad \text { for } \quad k \in \mathbb{Z} .
$$

By Lemma (6.14) and Assertion (6.16.2), $Z_{-k} A_{1} \geq 0$ and $Z_{-_{k}} A_{3} \geq 0$ for any $k \in \mathbb{Z}$. For $Z_{-k} A_{2}$, we have

$$
\begin{array}{ll}
Z_{-k} A_{2}=-3 m+4 m-m=0 & \text { if } \quad k \equiv 0(\bmod 4) \\
Z_{-k} A_{2}=-3 m-1+2(2 m+1)-(m+1)=0 & \text { if } \quad k \equiv 1(\bmod 4) \\
Z_{-k} A_{2}=-3 m-2+2(2 m+1)-(m+1)=-1 & \text { if } \quad k \equiv 2(\bmod 4) \\
Z_{-k} A_{2}=-3 m-3+2(2 m+2)-(m+1)=0 & \text { if } \quad k \equiv 3(\bmod 4)
\end{array}
$$

Hence $Z_{-k}=L_{-k}$ if $k \neq 2(\bmod 4)$ by $(6.12)$. In case $k \equiv 2(\bmod 4)$, we set $Z_{-k}-A_{2}=$ $Z_{-k}^{(1)}$. Then $Z_{-k}^{(1)} A_{2}=1$, and $Z_{-k}^{(1)}=L_{-k}$ in this case. Therefore we obtain the followings:

$$
\begin{aligned}
& L_{-k}=-k E-\left\{\frac{3}{4} k\right\} A_{1}-\left\{\frac{2}{4} k\right\} A_{2}-\left\{\frac{1}{4} k\right\} A_{3} \quad \text { if } \quad k \neq 2(\bmod 4), \\
& L_{-k}=-k E-\left\{\frac{3}{4} k\right\} A_{1}-\left(\left\{\frac{2}{4} k\right\}+1\right) A_{2}-\left\{\frac{1}{4} k\right\} A_{3} \quad \text { if } \quad k \equiv 2(\bmod 4) .
\end{aligned}
$$

Example (6.19). Let the situation be as in Theorem (6.3) and the dual graph of $f^{-1}(w)$ be as follows:

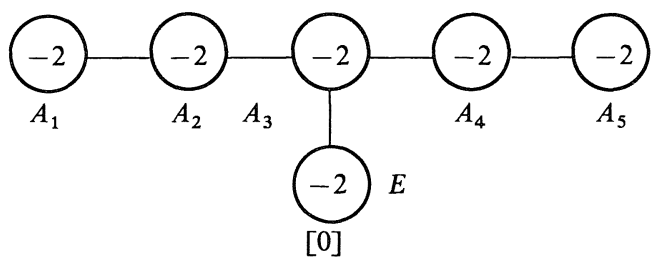

Here $A_{1} \cup A_{2} \cup A_{3} \cup A_{4} \cup A_{5}$ is contracted to a normal point by $\tau: \tilde{X} \rightarrow X$. Let us define the filtration $\left\{\bar{F}^{k}\right\}_{k \in \mathbb{Z}}$ on $A$ by $\bar{F}^{k}=\psi_{*}\left(O_{X}(-k E)\right)$ and study $\bigoplus_{k \geq 0}\left(\bar{F}^{k} / \bar{F}^{k+1}\right)$ by the technique of the proof of Theorem (6.3). Let the divisor $L_{-k}$ on $\tilde{X}$ be Giraud's inverse image of Weil divisor $-k \cdot E$ on $X$, for $k \in \mathbb{Z}$. If we denote $-k E-\left\{e_{\tau}(-k E)\right\}$ 
by $Z_{-k}$ on $\tilde{X}$, we have

$$
Z_{-k}=-k E-\left\{\frac{k}{2}\right\} A_{1}-k A_{2}-\left\{\frac{3}{2} k\right\} A_{3}-k A_{4}-\left\{\frac{k}{2}\right\} A_{5} .
$$

We can see the behaviour of $Z_{-k} A_{i}$ :

$$
\begin{aligned}
& Z_{-k} A_{1}=Z_{-k} A_{3}=Z_{-k} A_{5}=2\left(\left\{\frac{k}{2}\right\}-\frac{k}{2}\right) \geq 0 . \\
& Z_{-k} A_{2}=Z_{-k} A_{4}=2\left(\frac{k}{2}-\left\{\frac{k}{2}\right\}\right) \leq 0 .
\end{aligned}
$$

Hence $Z_{-k}=L_{-k}$ when $k$ is even, and $L_{-k}=Z_{-k}-A_{2}-A_{3}-A_{4}$ when $k$ is odd. Let the effective divisor $W_{-k}$ be defined as follows: $W_{-k}=L_{-k}-L_{-k-1}-\mathbb{E}$ for $k \in \mathbb{Z}$. Then we can easily check the conditions: $p_{a}\left(W_{-k}\right)-1+W_{-k} E-W_{-k} L_{-k}=0$ for $k \in \mathbb{Z}$. Therefore $O_{X}(-k E) / O_{X}(-(k+1) E)=\tau_{*}\left(O_{E} \otimes O_{\tilde{X}}\left(\mathbb{L}_{-k}\right)\right)$ follow for $k \in \mathbb{Z}$. Since $H^{1}\left(E, O_{E} \otimes O_{\tilde{X}}\left(L_{-k}\right)\right)=0$ for $k \geq 0$, we obtain the isomorphism

$$
\bigoplus_{k \geq 0}\left(\bar{F}^{k} / \bar{F}^{k+1}\right) \cong \bigoplus_{k \geq 0} H^{0}\left(E, O_{E} \otimes O_{\tilde{X}}\left(\mathbb{L}_{-k}\right)\right)
$$

Further one can easily see the isomorphism

$$
\bigoplus_{k \geq 0}\left(\bar{F}^{k} / \bar{F}^{k+1}\right) \cong k[x, y, z] /\left(x^{2}+y^{3}\right) \quad \text { (cf. Example (2.5)) }
$$

Remark (6.20). The inequality $p_{g}(A) \leq p_{g}(\mathbb{R}(E, \mathbb{D}))$ had already been pointed out in Corollary (2.22) [47]. This fact is used implicitly in the proof of (2.3) [27].

\section{§7. Gorenstein Singularities with "Star-Shaped" Resolution}

(7.1) Let the situation be as in Theorem (6.3). In this section, we shall study the relationship of Gorenstein property of $A, G$ and $R(E, D)$. Then we will study $U \cong$ $H_{G_{+}}^{1}(G)$ and the morphisms $H^{1}\left(X, O_{X}(n)\right) \rightarrow H_{m}^{2}(A)$ in the case $A$ is a Gorenstein domain. First we characterize Gorenstein property of $A$ in terms of the morphisms above and Gorenstein property of $R(E, D)$ in the following.

Theorem (7.2). Let the situation be as in (6.2). Then the following two conditions are equivalent.

(1) $(A, m)$ is a Gorenstein local domain.

(2) $R(E, D)$ is a Gorenstein domain, and the canonical restriction map

$$
H^{1}\left(X, O_{X}(-a E)\right) \cong R^{1} \psi_{*}\left(O_{X}(-a E)\right) \rightarrow H^{1}\left(X-E, O_{X}(-a E)\right) \cong H_{m}^{2}(A),
$$

induced from inclusion $X-E \rightarrow X$, is injective. Here $a=a(R(E, D))$.

Remark (7.3). The criterions in [48] of Gorenstein property for elliptic singularities are in some sense analogous to ours. 
The following argument shows that the latter half of the second condition of Theorem (7.2) is essential.

Remark (7.4) ([19], [20]). Let the situation be as in Example (6.9). Suppose the singularity $(W, w)$ is Gorenstein and $\xi$ is not zero in $H^{1}(E, L)$. Then:

(1) If the characteristic of base field is zero, $a(R)$ is one, i.e., $L \cong K_{E}$.

(2) If the characteristic of base field is positive, say $p$, then $a(R)$ is positive and $p$ divides $a(R)-1$.

(The proof is contained in page 153 of [19]. See [21] for a proof by using our Theorem (7.2).)

Conversely, in Example (6.9), if $L \cong K_{E}$, then $U=0$ and $(W, w)$ is Gorenstein by Corollary (6.6) (see also [19], [20]).

Remark (7.5). Let the situation be as in Example (6.9). Suppose the characteristic of base field is zero and $\xi \neq 0$. If the relation $L^{a} \cong K_{E}$ holds for some integer $a \geq 2$, then $(W, w)$ is not Gorenstein by Theorem (7.4) though $R(E, L)$ is a Gorenstein domain [45].

Concerning the theorem and remarks in the above, we will propose the following:

Problem (7.6.1)*. When $(A, m)$ is a Gorenstein local ring with "star-shaped" resolution, does our $U$ always vanish?

By Theorem (6.3), this is equivalent to the following (cf. (5.16)).

Problem (7.6.2). When $(A, m)$ is a Gorenstein local ring with "siar-shaped" resolution, is $(A, m)$ a $p_{g}$-constant small deformation of $\left(\operatorname{Spec}\left(R(E, D), R_{+}\right)\right.$?

By Theorem (5.6), this is equivalent to the following.

Problem (7.6.3). Let $(A, m)$ be a normal two-dimensional Gorenstein domain over an algebraically closed field with filtration $\left\{F^{k}\right\}_{k \in \mathbf{Z}}$ on $A$ such that $G=\bigoplus_{k \geq 0}\left(F^{k} / F^{k+1}\right)$ is an integral domain with isolated singularity. Then, is $G$ normal (i.e., Cohen-Macaulay, or equivalently Gorenstein in this case)?

Up to now, we do not know a counterexample to them. In this section and Section 8 , we shall show some partial affirmative answers to them.

In the proof of Theorem (7.2), we will obtain the following (see (7.12)):

Corollary (7.7). Let the situation be as in (6.2). If $(A, m)$ is Gorenstein with $a(R(E, D)) \leq 2$, then $U=0$.

Then after some lemmas on generality of $U$, we will show in (7.18):

Theorem (7.8). Let the situation be as in (6.3). Suppose the characteristic of the base field $k$ is not 2. If $(A, m)$ is Gorenstein, then $\operatorname{dim} U \neq 1$.

To state Theorem (7.9), we use the partial resolution of $\operatorname{Spec}(R(E, D))$ in the following: Let $v:(C, E)=\left(\operatorname{Spec}_{E}\left(\bigoplus_{k \geq 0} O_{E}(k D)\right), E\right) \rightarrow\left(\operatorname{Spec}(R(E, D)), p=V\left(m_{R}\right)\right)$ be the

\footnotetext{
* See Note Added in Proof (2).
} 
partial resolution of $(\operatorname{Spec}(R(E, D)), p)$ obtained by the filtration on $R(E, D)$ induced by the grading on $R(E, D)$ (cf. Sections 1, 5, and 6, and [34], [7], [32], [33]).

Theorem (7.9). Let the situation be as in (6.2). Suppose the characteristic of the base field $k$ is zero. If $(A, m)$ is Gorenstein with $\operatorname{dim}\left(m_{R} \cdot R^{1} v_{*}\left(O_{C}\right) /\left(m_{R}\right)^{2} \cdot R^{1} v_{*}\left(O_{C}\right)\right) \leq 1$, then $U=0$.

Here recall the inequalities for the arithmetic genus $p_{a}(\Gamma)$ of the dual graph $\Gamma$ :

$$
\operatorname{dim}\left(\frac{\left(m_{R}\right)^{k} \cdot R^{1} v_{*}\left(O_{C}\right)}{\left(m_{R}\right)^{k+1} \cdot R^{1} v_{*}\left(O_{C}\right)}\right) \leq p_{a}(\Gamma) \quad \text { for } \quad k \geq 0
$$

(see Theorem (3.4) and the arguments in (3.3) of [42]). See (0.7) for the definition, and [43], [41], [42] for the arguments about the arithmetic genus.

Corollary (7.10). Let the situation be as in (6.2). Suppose the characteristic of the base field $k$ is zero. If $(A, m)$ is Gorenstein with $p_{a}(\Gamma) \leq 1$, then $U=0$.

In the rest of this section, we prove Theorem (7.2), Corollary (7.7), Theorem (7.8), and Theorem (7.9). We shall begin with the following:

Lemma (7.11). Let the situation be as in (6.2). Suppose $(A, m)$ is Gorenstein. Let $a=a(R)$ where $R=R(E, D)$ and $n$ an integer with $0 \leq n \leq a+1$. Then we have the duality of k-vector spaces:

$$
\begin{aligned}
\operatorname{Ker}\left\{R^{1} \psi_{*}\left(O_{X}(-n \cdot E)\right) \longrightarrow\right. & \left.R^{1} \psi_{*}\left(O_{X}\right)\right\} \\
\stackrel{\text { dual over } k}{\longrightarrow} & \operatorname{Ker}\left\{R^{1} \psi_{*}\left(O_{X}(-(a+1-n) \cdot E)\right) \longrightarrow R^{1} \psi_{*}\left(O_{X}\right)\right\} .
\end{aligned}
$$

Proof. By Theorem (3.12), the canonical sheaf $\omega_{X}$ of $X$ is written in the form $O_{X}(-m E)$ with the integer $m$ satisfying the relation $R(m-1) \cong K_{R}$, and $R$ is Gorenstein. Here $m-1$ equals $a(R)$. From the relations $\omega_{X} \cong O_{X}(-(a+1) \cdot E)$ and $\omega_{W} \cong A$, we obtain $\mathbf{R H o m}_{X}\left(\mathbf{R} \psi_{*}\left(O_{X}(-n \cdot E), \omega_{X}\right) \cong O_{X}(-(a+1-n) E)\right.$. By the relative duality

$$
\mathbb{R H o m}_{W}\left(\mathbb{R} \psi_{*}(\mathscr{F}), \omega_{W}^{\cdot}\right) \cong \mathbb{R} \psi_{*}\left(\operatorname{RHom}_{X}\left(\mathscr{F}, \omega_{X}^{\circ}\right)\right)
$$

for $\psi: X \rightarrow W$ and $\mathscr{F}=O_{X}(-n \cdot E)$, we obtain the following spectral sequence:

$$
\begin{aligned}
E_{2}^{p, q} & =\operatorname{Ext}_{A}^{p}\left(R^{-q} \psi_{*}\left(O_{X}(-n \cdot E)\right), A\right) \\
& \Rightarrow E^{p+q}=R^{p+q} \psi_{*}\left(O_{X}(-(a+1-n) E)\right) .
\end{aligned}
$$

Then we obtain the following exact sequence

$$
0 \rightarrow E^{0} \rightarrow E_{2}^{0,0} \rightarrow E_{2}^{2,-1} \rightarrow E^{1} \rightarrow E_{2}^{1,0} \rightarrow 0 .
$$

Here $E_{2}^{0,0}=A$ and $E_{2}^{1,0}=\operatorname{Ext}_{A}^{2}\left(A / F^{n}, A\right)$, since $A$ is a Cohen-Macaulay integral domain. Let $\mathrm{E}(A / m)$ be the injective hull of $A / m$. Then we obtain the equality $\operatorname{Ext}_{A}^{2}(\mathscr{G}, A)=$ $\operatorname{Hom}_{A}(\mathscr{G}, \mathrm{E}(A / m))$, which we shall denote by $[\mathscr{G}]^{\prime}$, for an Artinian $A$-module $\mathscr{G}$ (Proposition (4.13) of [14]). Since $R^{1} \psi_{*}\left(O_{X}(-(a+1) E)\right)=0$ by Grauert-Riemenschneider vanishing theorem $([13],[10])$, we obtain the following: 


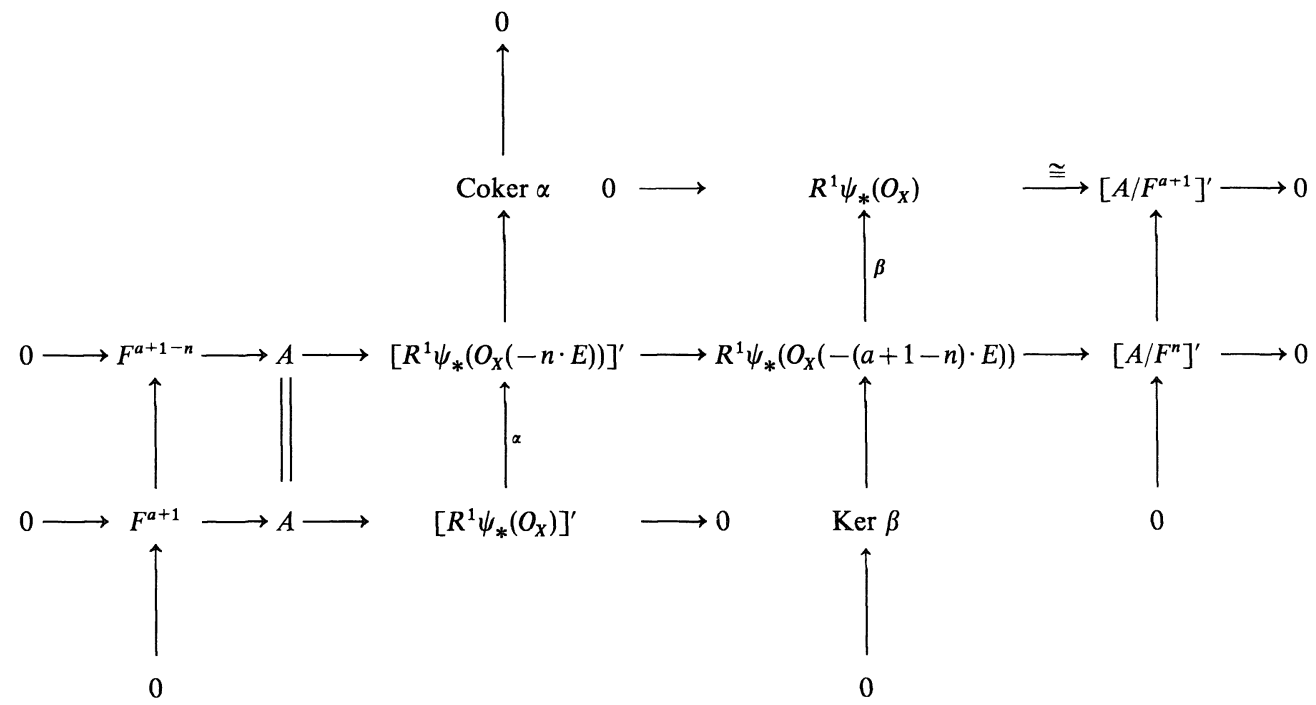

Now it is easy to see the isomorphism Coker $\alpha \cong \operatorname{Ker} \beta$.

Q.E.D.

(7.12) Proof of the implication of (2) from (1) in Theorem (7.2). As we have noted in (7.11), $R(E, D)$ is Gorenstein. Since $R^{1} \psi_{*}\left(O_{X}(-E)\right) \rightarrow R^{1} \psi_{*}\left(O_{X}\right)$ is injective by (6.5), $R^{1} \psi_{*}\left(O_{X}(-a \cdot E)\right) \rightarrow R^{1} \psi_{*}\left(O_{X}\right)$ is also injective by Lemma (7.11).

By the Grauert-Riemenschneider vanishing theorem $([13],[10])$ and the relative duality (see p. 188 of [29]), $H_{E}^{1}\left(X, O_{X}\right) \cong\left[R^{1} \psi_{*}\left(\omega_{X}\right)\right]^{\prime}=0$ and $R^{1} \psi_{*}\left(O_{X}\right) \rightarrow$ $H^{1}\left(X-E, O_{X}\right) \cong H_{m}^{2}(A)$ is injective. Therefore $R^{1} \psi_{*}\left(O_{X}(-a \cdot E)\right) \rightarrow H_{m}^{2}(A)$ is injective.

(7.13) Proof of the implication of (1) from (2) in Theorem (7.2). Here we shall use the notation in Section 6. Let us consider the minimal good resolution $f$ (resp. $\mu$ ) and the partial resolution $\varphi$ (resp. $v)$ of the singularity $(W, w)\left(\operatorname{resp} .\left(\operatorname{Spec}\left(R(E, D), V\left(R_{+}\right)=p\right)\right)\right.$ in the following:
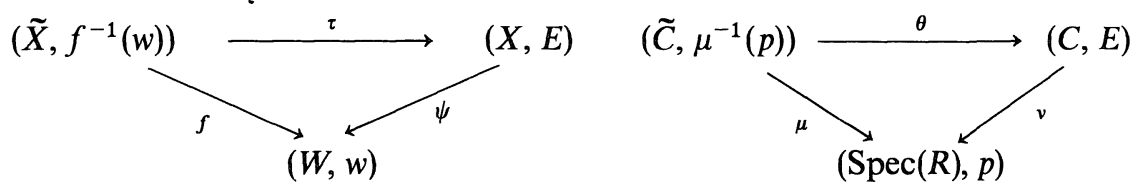

where $\tau$ (resp. $\theta$ ) is the contraction of the $\beta$ branches of resolution manifold $\tilde{X}$ (resp. $\tilde{C}$ ) (cf. (6.1)). Since $R(E, D)$ is Gorenstein, there is an integral divisor, say $K_{\tilde{C}}$, on $\widetilde{C}$ whose support is contained in $\operatorname{Supp}\left(\mu^{-1}(p)\right)$ and $\Omega_{\tilde{C}}^{2} \cong O_{\tilde{C}}\left(K_{\tilde{C}}\right)$. Here the condition $R^{1} \theta_{*} O_{\tilde{C}}=0$ implies the relations $\theta_{*}\left(O_{\tilde{C}}\left(K_{\tilde{C}}\right)\right)=\omega_{c} \cong O_{C}(-(a+1) \cdot E)$ on $C$. Denote Giraud's inverse image of $-(a+1) \cdot E$ by $L_{-a-1}$ on $\tilde{C}$. Then we obtain the inequality $K_{\tilde{C}} \geq L_{-a-1}$ on $\tilde{C}$. We have $e_{\theta}\left(K_{\tilde{C}}\right) \ll 0$, because $\theta$ is induced by the minimal resolution. Hence $0 \leq K_{\tilde{\mathbf{C}}}-L_{-a-1}=\left[K_{\tilde{\mathbf{C}}}-L_{-a-1}\right]_{\mathrm{G}}=\left[K_{\tilde{\mathbf{C}}}-L_{-a-1}-e_{\theta}\left(L_{-a-1}\right)\right]=\left[e_{\theta}\left(K_{\tilde{\mathbf{C}}}\right)\right]_{\mathrm{G}} \leq 0$. Therefore $K_{\tilde{C}}=L_{-a-1}$ on $\tilde{C}$.

Now we shall compare $\Omega_{\tilde{X}}^{2}$ with Giraud's inverse image of $-(a+1) \cdot E$ on $\tilde{X}$ by $\tau$. Here the dual graph of $f^{-1}(w)$ with respect to $\tilde{X}$ and the dual graph of $\mu^{-1}(p)$ with respect to $\tilde{C}$ are the same. Hence Giraud's inverse image of $-k \cdot E$ on $\tilde{X}$ by $\tau$ and 
Giraud's inverse image of $-k \cdot E$ on $\tilde{C}$ by $\theta$ have the same multiplicity on each components of supports. We shall denote both divisors uniformly by $\mathbb{L}_{-k}$.

Clearly $e_{f}\left(L_{-a-1}\right)=e_{f}\left(\Omega_{\tilde{X}}^{2}\right)$ in $N S(f, \mathbb{Z})$ (i.e., numerically the same). We shall show the relation $\Omega_{\tilde{X}}^{2} \cong O_{\tilde{X}}\left(L_{-a-1}\right)$. Let us set $B=E \cup\left(\bigcup_{i, j} A_{i j}\right) \subset \tilde{X}$. We have the following exact sequences:

$$
0 \rightarrow H^{1}\left(\tilde{X}, O_{X}\left(L_{-a}\right)\right) \rightarrow H^{1}\left(\tilde{X}-B, O_{\tilde{X}}\left(L_{-a}\right)\right) \rightarrow H_{B}^{2}\left(\tilde{X}, O_{\tilde{X}}\left(L_{-a}\right)\right) \rightarrow 0,
$$

and

$$
H^{1}\left(\tilde{X}, O_{\tilde{X}}\left(L_{-a-1}\right)\right) \rightarrow H^{1}\left(\tilde{X}-B, O_{\tilde{X}}\left(L_{-a-1}\right)\right) \rightarrow H_{B}^{2}\left(\tilde{X}, O_{\tilde{X}}\left(L_{-a-1}\right)\right) \rightarrow 0
$$

where $H^{1}\left(\tilde{X}, O_{\tilde{X}}\left(L_{-a-1}\right)\right) \cong R^{1} \psi_{*}\left(O_{X}(-(a+1) E)\right)=0$ (since $H^{1}\left(O_{E}(k D)\right)=0$ for $\left.k \geq a+1\right)$ and $H^{1}\left(\tilde{X}-B, O_{\tilde{X}}\left(L_{-a}\right)\right) \cong H^{1}\left(\tilde{X}-B, O_{\tilde{X}}\left(L_{-a-1}\right)\right) \cong H_{m}^{2}(A)$ (see Sections 1, 5, and 6). Taking the dual over $\mathbb{E}(A / m)$ (= the injective hull of $A / m)$, we obtain the following exact sequence:

$$
\begin{aligned}
0 & \rightarrow\left[H^{0}\left(\tilde{X}, O_{\tilde{X}}\left(K_{\tilde{X}}-L_{-a}\right)\right)\right]^{\wedge} \cong\left[\psi_{*}\left(O_{\tilde{X}}\left(K_{\tilde{X}}-L_{-a}\right)\right)\right]^{\wedge} \\
& \rightarrow\left[H^{0}\left(\tilde{X}, O_{\tilde{X}}\left(K_{\tilde{X}}-L_{-a-1}\right)\right)\right]^{\wedge} \cong\left[\psi_{*}\left(O_{\tilde{X}}\left(K_{\tilde{X}}-L_{-a-1}\right)\right)\right]^{\wedge} \cong\left(K_{A}\right)^{\wedge} \\
& \rightarrow\left[H^{1}\left(\tilde{X}, O_{\tilde{X}}\left(L_{-a}\right)\right)\right]^{\prime} \rightarrow 0,
\end{aligned}
$$

where $(\mathscr{F})^{\wedge}$ denote the $m$-adic completion of an $A$-module $\mathscr{F}$ (see p. 188 of [29]). Since $H^{1}\left(\tilde{X}, O_{\tilde{X}}\left(L_{-a}\right)\right) \cong H^{1}\left(E, O_{E}(a \cdot D)\right)$ is not zero, the natural injection $H^{0}\left(\tilde{X}, O_{\tilde{X}}\left(K_{\tilde{X}}-L_{-a}\right)\right) \rightarrow$ $H^{0}\left(\tilde{X}, O_{\tilde{X}}\left(K_{\tilde{X}}-L_{-a-1}\right)\right)$ is not surjective. There is a meromorphic two-form $\beta$ on $\tilde{X}$ which is holomorphic on $\tilde{X}-B$ and does not belong to $H^{0}\left(\tilde{X}, O_{\tilde{X}}\left(K_{\tilde{X}}-L_{-a}\right)\right)$. We represent the $\operatorname{divisor} \operatorname{div}(\beta)$ as: $\operatorname{div}(\beta)=B_{1}(\beta)+B_{2}(\beta)$ where $\operatorname{Supp}\left(B_{1}(\beta)\right) \subset B, B_{2}(\beta)$ is effective and has no common irreducible component with $B$. By assumption $B_{1}(\beta) \geq L_{-a}$. Since $e_{f}(\operatorname{div}(\beta))=e_{f}\left(\Omega_{\tilde{X}}^{2}\right)=e_{f}\left(L_{-a-1}\right), e_{f}\left(B_{1}(\beta)-L_{-a-1}\right)=e_{f}\left(-B_{2}(\beta)\right) \gg 0$ and $B_{1}(\beta)-L_{-a-1} \geq 0$.

\section{Assertion (7.13.1)。 $\quad B_{1}(\beta)=L_{-a-1}$.}

Otherwise $B_{1}(\beta)-L_{-a-1}>0$. Then, by definition, $B_{1}(\beta)-L_{-a-1} \geq$ Artin's fundamental cycle $\mathbb{Z}_{0}$ on $B$. We can easily see $\mathbb{Z}_{0} \gg L_{-1}=-E-\left[e_{\tau}(-E)\right]_{G}$. Further $L_{-a-1} \geq$ $L_{-1}+L_{-a}$ by a characteristic of Giraud's []$_{G}[10]$. Therefore $B_{1}(\beta) \geq L_{-a}$. However this contradicts our assumption on $\beta$.

Hence $\operatorname{div}(\beta)=L_{-a-1}$ and $\Omega_{\tilde{X}}^{2} \cong O_{\tilde{X}}\left(L_{-a-1}\right)$.

This completes the proof of Theorem (7.2).

(7.14) We shall discuss the generalities of our $U \cong H_{G_{+}}^{1}(G)$ which are used in the proof of Theorems (7.8) and (7.9).

Let the situation be as in (6.2). Let $\eta_{i, j}$ with $i>j$ and $\xi_{p}$ with $p \geq 0$ denote the following canonical morphisms:

$$
\begin{aligned}
\eta_{i, j}: R^{1} \psi_{*}\left(O_{X}(-i \cdot E)\right) & \rightarrow R^{1} \psi_{*}\left(O_{X}(-j \cdot E)\right) \quad \text { with } i>j \\
\xi_{p}: R^{1} \psi_{*}\left(O_{X}(-p \cdot E)\right) & \rightarrow H^{1}\left(E, O_{E}(p \cdot D)\right)
\end{aligned}
$$


Then $U=\bigoplus_{k \geq 0} U_{k}=\bigoplus_{k \geq 0} \operatorname{Ker} \eta_{k+1, k}$. Let $\Phi$ be a non-zero element of $U_{k}$. Then one can easily see the existence of an integer $p$ and an element $\Psi$ of $R^{1} \psi_{*}\left(O_{X}(-p \cdot E)\right)$ satisfying the following conditions: $p \geq k+1, \eta_{p, k}(\Psi)=\Phi$ in $R^{1} \psi_{*}\left(O_{X}(-k \cdot E)\right)$, and $\xi_{p}(\Psi) \neq 0$ in $H^{1}\left(E, O_{E}(p \cdot D)\right)$. Such a couple $(p, \Psi)$ is not unique for $\Phi$ of $U_{k}$, in general. For $(p, \Psi)$, we shall prove the following:

Lemma (7.15). Let $\Phi$ and $(p, \Psi)$ be as above. Let $y$ be an element of $R_{k}=$ $H^{0}\left(E, O_{E}(k \cdot D)\right)$ corresponding to $\Phi$ by $R_{k} \rightarrow U_{k}$. Suppose $t y^{t-1} \xi_{p}(\Psi)$ is not zero in $H^{1}\left(E, O_{E}(((t-1) k+p) \cdot D)\right)$ for an integer $t \geq 1$.

Then there is a non-zero element $\Phi^{(t)}$ in

$$
\operatorname{Ker} \eta_{(t-1) k+p, t k}=\operatorname{Ker}\left\{R^{1} \psi_{*}\left(O_{X}(-((t-1) k+p) \cdot E)\right) \rightarrow R^{1} \psi_{*}\left(O_{X}(-t \cdot k \cdot E)\right)\right\}
$$

such that

$$
\left.\xi_{(t-1) k+p}\left(\Phi^{(t)}\right)=t y^{t-1} \xi_{p}(\Psi) \quad \text { in } \quad H^{1}\left(E, O_{E}((t-1) k+p) D\right)\right) .
$$

Proof. Let $\mathscr{U}=\left\{\mathscr{U}_{i}\right\}_{i \in \mathscr{I}}$ be an affine open covering of $X$ with index set $\mathscr{I}$. We shall construct an element $\Phi^{(t)}$ in Čech-cohomology. Take a zero-cochain $\tilde{y}=\left\{\tilde{y}_{i}\right\}_{i \in \mathscr{I}}$ of $\check{C}^{0}\left(\mathscr{U}, O_{X}(-k E)\right)$ which is mapped to $y$ as follows:

$$
\begin{gathered}
H^{0}\left(\mathscr{U}_{i}, O_{X}(-k E)\right) \longrightarrow H^{0}\left(\mathscr{U}_{i}, O_{E}(k D)\right) \longrightarrow 0 \\
u \\
\left.\tilde{y}_{i} \longrightarrow y\right|_{\mathscr{U}_{i}}=\text { the restriction of } y \text { to } \mathscr{U}_{i} .
\end{gathered}
$$

Then one-cocycle $\left\{\tilde{y}_{j}-\tilde{y}_{i}\right\}_{i, j \in \mathscr{I}}$ represents $\Phi$ of $\check{H}^{1}\left(\mathscr{U}, O_{X}(-(k+1) \cdot E)\right)$. By assumption we can take $\left\{\tilde{y}_{i}\right\}_{i \in \mathscr{I}}$ so that

(7.15.1) $\tilde{y}_{j}-\tilde{y}_{i} \in H^{0}\left(\mathscr{U}_{j} \cap \mathscr{U}_{i}, O_{X}(-p \cdot E)\right)$ for $i, j \in \mathscr{I}$ and the cocycle $\left\{\tilde{y}_{j}-\tilde{y}_{i}\right\}$ represents $\Psi$ of $\check{H}^{1}\left(X, O_{X}(-p \cdot E)\right)$.

For the one-cocycle $\left\{\left(\tilde{y}_{j}\right)^{t}-\left(\tilde{y}_{i}\right)^{t}\right\}_{i, j \in \mathscr{I}}$ of $\check{Z}^{1}\left(\mathscr{U}, O_{X}(-t \cdot k \cdot E)\right)$, we have the relations

$$
\left(\tilde{y}_{j}\right)^{t}-\left(\tilde{y}_{i}\right)^{t}=\sum_{s=1}^{t}\left(\begin{array}{l}
t \\
s
\end{array}\right)\left(\tilde{y}_{i}\right)^{t-s}\left(\tilde{y}_{j}-\tilde{y}_{i}\right)^{s} \quad \text { on } \quad \mathscr{U}_{i} \cap \mathscr{U}_{j}
$$

where $\left(\begin{array}{l}t \\ s\end{array}\right)$,s denote the binomial coefficients, $i, j \in \mathscr{I}$,

$$
\begin{gathered}
\left(\tilde{y}_{i}\right)^{t-1}\left(\tilde{y}_{j}-\tilde{y}_{i}\right) \in H^{0}\left(\mathscr{U}_{i} \cap \mathscr{U}_{j}, O_{X}(-((t-1) k+p) \cdot E)\right), \quad \text { and } \\
\left(\tilde{y}_{i}\right)^{t-s}\left(\tilde{y}_{j}-\tilde{y}_{i}\right)^{s} \in H^{0}\left(\mathscr{U}_{i} \cap \mathscr{U}_{j}, O_{X}(-((t-1) k+p+1) \cdot E)\right)
\end{gathered}
$$

for $s>1$ and $i, j \in \mathscr{I}$.

Hence $\left\{\left(\tilde{y}_{j}\right)^{t}-\left(\tilde{y}_{i}\right)^{t}\right\} \in \check{Z}^{1}\left(\mathscr{U}, O_{X}(-((t-1) k+p) \cdot E)\right)$, and defines an element of $R^{1} \psi_{*}\left(O_{X}(-((t-1) k+p) \cdot E)\right)$. We denote it by $\Phi^{(t)}$. We can easily see that $\Phi^{(t)}$ has the desired properties.

Next we shall show the following:

Lemma (7.16). Let the situation be as in (6.2) and (7.14). Then we have the 
equality

$$
\operatorname{dim} U=\sum_{k \geq 1} \operatorname{dim}\left(\xi_{k}\left(\operatorname{Ker}\left\{R^{1} \psi_{*}\left(O_{X}(-k \cdot E)\right) \rightarrow R^{1} \psi_{*}\left(O_{X}\right)\right\}\right)\right)
$$

Proof. Take the subvector spaces $W_{k}$ of $R^{1} \psi_{*}\left(O_{X}(-k \cdot E)\right), k \geq 1$, with following properties: $W_{k} \subset \operatorname{Ker} \eta_{k, 0}$ and the restriction $\left.\xi_{k}\right|_{W_{k}}: W_{k} \rightarrow \xi_{k}\left(\operatorname{Ker} \eta_{k, 0}\right)$ is isomorphic, i.e., injective and surjective, for $k \geq 0$. Then the surjectivity of $\xi_{k}$ 's imply the relations;

$$
U_{\alpha} \subset \sum_{k \geq \alpha+1} \eta_{k, \alpha+1}\left(W_{k}\right) \quad \text { in } \quad R^{1} \psi_{*}\left(O_{X}(-(\alpha+1) \cdot E)\right) \quad \text { for } \quad \alpha \geq 0 .
$$

Further we can easily show the relations:

$$
\left(\sum_{k \geq \alpha+1} \eta_{k, \alpha}\left(W_{k}\right)\right) \cap W_{\alpha}=0 \quad \text { for } \quad \alpha \geq 1 \text {. }
$$

Hence we obtain

$$
\begin{aligned}
\operatorname{dim} U & =\sum_{\alpha \geq 0} \operatorname{dim}\left(U_{\alpha} \cap\left(\sum_{k \geq \alpha+1} \eta_{k, \alpha+1}\left(W_{k}\right)\right)\right) \\
& =\sum_{\alpha \geq 0}\left(\operatorname{dim}\left(\sum_{k \geq \alpha+1} \eta_{k, \alpha+1}\left(W_{k}\right)\right)-\operatorname{dim}\left(\sum_{k \geq \alpha+1} \eta_{k, \alpha}\left(W_{k}\right)\right)\right) \\
& =\sum_{\alpha \geq 1} \operatorname{dim} W_{\alpha} .
\end{aligned}
$$

Lemma (7.17). Let the situation be as in (6.2) and (7.14). Suppose (W,w) is Gorenstein. Then

$$
\begin{gathered}
\text { Image }\left\{F^{k} \rightarrow R_{k}\right\}=\left\{x \in R_{k} \mid x \cdot \xi_{a-k}\left(\operatorname{Ker}\left\{R^{1} \psi_{*}\left(O_{X}(-(a-k) \cdot E)\right) \rightarrow R^{1} \psi_{*}\left(O_{X}\right)\right\}\right)\right. \\
\left.=0 \quad \text { in } H^{1}\left(E, O_{E}(a D)\right)\right\}
\end{gathered}
$$

for $k \geq 0$, where $a=a(R(E, D))$.

Proof. In general

$$
\begin{aligned}
F^{k} \cdot \operatorname{Ker} & \left\{R^{1} \psi_{*}\left(O_{X}(-(a-k) \cdot E)\right) \rightarrow R^{1} \psi_{*}\left(O_{X}\right)\right\} \\
& \subset \operatorname{Ker}\left\{R^{1} \psi_{*}\left(O_{X}(-a \cdot E)\right) \rightarrow R^{1} \psi_{*}\left(O_{X}(-k \cdot E)\right)\right\} .
\end{aligned}
$$

By Theorem (7.2) and (7.12), these are zero in our case. Hence we can show the following relations:

$$
\begin{array}{r}
\text { Image }\left\{F^{k} \rightarrow R_{k}\right\} \subset\left\{x \in R_{k} \mid x \xi_{a-k}\left(\operatorname{Ker} \eta_{a-k, 0}\right)=0 \text { in } H^{1}\left(E, O_{E}(a D)\right)\right\} \\
\text { for } k \geq 0 .
\end{array}
$$

By the Serre duality $R_{k} \leftrightarrow H^{1}\left(E, O_{E}((a-k) D)\right)$ (cf. [45]), $\left\{x \in R_{k} \mid x \xi_{a-k}\left(\operatorname{Ker} \eta_{a-k, 0}\right)=\right.$ $0\}$ is the dual of $H^{1}\left(E, O_{E}((a-k) D)\right) / \xi_{a-k}\left(\operatorname{Ker} \eta_{a-k, 0}\right)$. Therefore we obtain

$$
\begin{aligned}
\operatorname{dim} U_{k} & =\operatorname{dim} R_{k}-\operatorname{dim}\left(\operatorname{Image}\left\{F^{k} \rightarrow R_{k}\right\}\right) \\
& \geq \operatorname{dim} R_{k}-\operatorname{dim}\left\{x \in R_{k} \mid x \cdot \xi_{a-k}\left(\operatorname{Ker} \eta_{a-k, 0}\right)=0\right\} \\
& =\operatorname{dim} \xi_{a-k}\left(\operatorname{Ker} \eta_{a-k, 0}\right) \quad \text { for } \quad k \geq 0 .
\end{aligned}
$$


Then the equalities in (7.17.2) follow from Lemma (7.16). Hence the equalities hold in (7.17.1).

Q.E.D.

Now the proof of Theorem (7.8) is a corollary of Theorem (7.2) and above Lemmas.

(7.18) Proof of Theorem (7.8). Suppose $\operatorname{dim} U=1$, say $U_{k} \neq 0$ here, and take a non-zero element $\Phi$ of $U_{k}$. Let $y \in R_{k}$ and $\Psi \in R^{1} \psi_{*}\left(O_{X}(-p \cdot E)\right)$ be as in (7.14) and Lemma (7.15).

Since $\operatorname{dim} U=1$, we obtain the equality $\bigoplus_{h \geq 1} \xi_{h}\left(\operatorname{Ker} \eta_{h, 0}\right)=k \xi_{p}(\Psi)$ by Lemma (7.16). Then, $p=a-k$ and $y \xi_{p}(\Psi) \neq 0$ in $H^{1}\left(E, O_{E}(a D)\right)$ by Lemma (7.17). Here $2 \cdot y \cdot \xi_{p}(\Psi) \neq 0$ in $H^{1}\left(E, O_{E}(a D)\right)$ because $\operatorname{char}(k) \neq 2$. Hence Ker $\eta_{a, 0}$ is non-trivial by Lemma (7.15). This is a contradiction to the fact $\operatorname{Ker} \eta_{a, 0}=0$ (Theorem (7.2) and (7.12)).

Q.E.D.

The rest of this section is devoted to the proof of Theorem (7.9).

(7.19) Proof of Theorem (7.9) in the case $m_{R} R^{1} v_{*}\left(O_{C}\right)=\left(m_{R}\right)^{2} R^{1} v_{*}\left(O_{C}\right)$. Then $m_{R} R^{1} v_{*}\left(O_{C}\right)=0$ by Nakayama's lemma. Since $R(E, D)$ is Gorenstein, $p_{g}(R) \leq 1$ by the proof of Corollary (2.10) of [42]. Hence $(W, w)$ is rational or minimally elliptic. In these cases, the vanishing of $U$ is classical ([2][26]).

(7.20) For the proof of remaining cases, we need some properties of $H_{R_{+}}^{2}(R)=$ $H^{1}\left(E, O_{E}(k \cdot D)\right) T^{k}$ where $R=R(E, D)$ (cf. [45], [12], [42]).

Let the situation be as in (6.2) and Theorem (6.3). Let $\lambda$ be a positive integer with $R_{\lambda}=H^{0}\left(E, O_{E}(\lambda D)\right) \neq 0$ and $x$ a non-zero element of $R_{\lambda}$. Since

$$
\operatorname{dim}\left(\operatorname{supp}\left(\frac{O_{E}((k+\lambda) \cdot D}{x \cdot O_{E}(k \cdot D)}\right)\right) \leq 0,
$$

the product with the element $x$

$$
x: H^{1}\left(E, O_{E}(k D)\right) \rightarrow H^{1}\left(E, O_{E}((k+\lambda) D)\right)
$$

is surjective for $k \in \mathbb{Z}$.

We shall use this fact freely in the below.

Let us consider the filtered blowing-up $v:(C, E)=\left(\operatorname{Spec}_{E}\left(\bigoplus_{k \geq 0} O_{E}(k D)\right), E\right) \rightarrow$ $\left(\operatorname{Spec}(R(E, D)), p=V\left(m_{R}\right)\right)$ (cf. Sections 1, 5, and 6). Let $\varepsilon=\varepsilon(R(E, D))$ be the positive integer as in (6.7). Then $m_{R}=R_{+}=\bigoplus_{k \geq \varepsilon} R_{k}$, and we have the following relations (see the proof of Theorem (3.4) of [42]):

$$
\frac{\left(m_{R}\right)^{k} R^{1} v_{*}\left(O_{C}\right)}{\left(m_{R}\right)^{k+1} R^{1} v_{*}\left(O_{C}\right)}=\sum_{h=k \cdot \varepsilon}^{(k+1) \cdot \varepsilon-1} H^{1}\left(E, O_{E}(h D)\right) \quad \text { for } \quad k \geq 0 .
$$

Henceforce we shall assume

$$
\operatorname{dim}\left(\frac{m_{R} R^{1} v_{*}\left(O_{C}\right)}{\left(m_{R}\right)^{2} R^{1} v_{*}\left(O_{C}\right)}\right)=1
$$


Let $\gamma \in\{0, \ldots, \varepsilon-1\}$ be the integer with $H^{1}\left(E, O_{E}((\varepsilon+\gamma) D)\right)=k$ and $x$ a non-zero element of $R_{\varepsilon}$. Then, by the surjectivity of (7.20.1),

$$
\begin{array}{lll}
\operatorname{dim} H^{1}\left(E, O_{E}(h D)\right)=0 & \text { if } \quad h \neq \gamma(\bmod \varepsilon) \quad \text { and } \quad h \geq \varepsilon, \\
\operatorname{dim} H^{1}\left(E, O_{E}(h D)\right) \leq 1 & \text { if } \quad h \equiv \gamma(\bmod \varepsilon) \quad \text { and } \quad h \geq \varepsilon .
\end{array}
$$

Hence there is a positive integer $v$ such that $a(R(E, D))=\gamma+v \cdot \varepsilon$. Then, we have the following commutative diagrams for integer $u$ with $1 \leq u \leq v$ :

$$
k=H^{1}\left(E, O_{E}((\varepsilon+\gamma) D)\right) \underset{\substack{\text { multiplication } \\ \text { with } x^{u-1}}}{\text { multiplication with } x^{v-1}} H_{H^{1}\left(E, O_{E}((\gamma+u \cdot \varepsilon) D)\right) .}^{H^{1}\left(E, O_{E}\left(D^{(a)}\right)\right)=k}
$$

Hence, by the surjectivity of (7.20.1), we obtain

$$
H^{1}\left(E, O_{E}((\gamma+u \cdot \varepsilon) D)\right)=k \quad \text { for } \quad u \quad \text { with } 1 \leq u \leq v
$$

$$
\text { the morphisms in the diagram (7.20.3) are isomorphic. }
$$

(7.21) We shall prove the remaining case by continuing the studies of (7.20) on assuming that $(W, w)$ is Gorenstein.

By (7.20.2), (7.20.4), and the Serre duality: $R_{\alpha} \leftrightarrow H^{1}\left(E, O_{E}((a-\alpha) D)\right)$ (cf. [45]),

$$
\begin{array}{llll}
R_{\alpha}=0 & \text { if } \quad \alpha \neq 0(\bmod \varepsilon) & \text { and } & 0 \leq \alpha \leq \gamma+(v-1) \cdot \varepsilon, \\
R_{\alpha}=k & \text { if } \quad \alpha \equiv 0(\bmod \varepsilon) & \text { and } & 0 \leq \alpha \leq \gamma+(v-1) \cdot \varepsilon .
\end{array}
$$

By the exact sequence $0 \rightarrow G \rightarrow R \rightarrow U \rightarrow 0$, we obtain the relations: $U_{\alpha}=0$ if $\alpha \neq \equiv$ $(\bmod \varepsilon)$ and $0 \leq \alpha \leq \gamma+(v-1) \cdot \varepsilon$. Further $U_{0}=0$ by (6.5).

Assertion (7.22)。 $U_{\varepsilon}=0$.

Proof. Suppose $U_{\varepsilon} \neq 0$ and take a non-zero element $\Phi$ of $U_{\varepsilon}$. Let $y \in R_{\alpha}, \Psi \in$ $R^{1} \psi_{*}\left(O_{X}(-p \cdot E)\right)$ be as in (7.14) and Lemma (7.15) in the following:

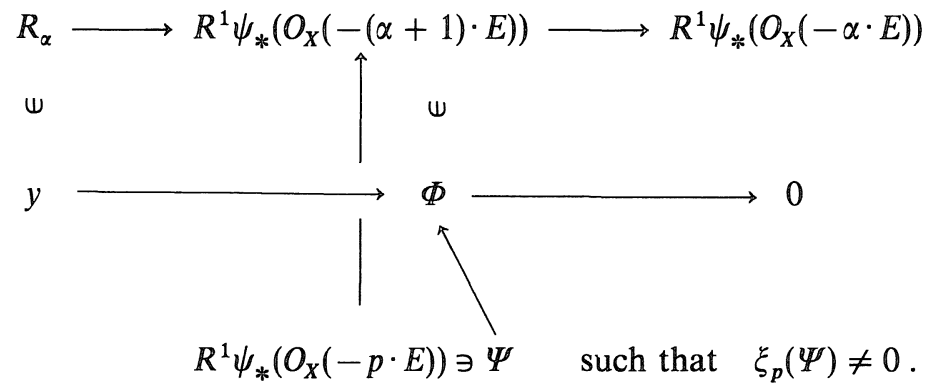

Then $p \equiv \gamma(\bmod \varepsilon)$ by (7.20.2). Let us write $p=\gamma+u \cdot \varepsilon$ with $1 \leq u \leq v$. Then $y^{u-v} \xi_{p}(\Psi) \neq 0$ in $H^{1}\left(E, O_{E}(a D)\right)$ by (7.19.5). Further $(u-v+1) \cdot y^{u-v} \cdot \xi_{p}(\Psi) \neq 0$ in $H^{1}\left(E, O_{E}(a D)\right)$, because char $(k)=0$. Hence $\operatorname{Ker} \eta_{a, 0}$ is non-trivial by Lemma (7.15). This is a contradiction by Theorem (7.2) and (7.12).

Therefore $U_{\varepsilon}=0$. 
(7.23) By assertion (7.22), there is an element $g$ of $F^{\varepsilon}-F^{\varepsilon+1}$ whose divisor $\operatorname{div}\left(\psi^{-1}(g)\right)$ is written as follows:

$\operatorname{div}\left(\psi^{-1}(g)\right)=-\varepsilon \cdot E+D_{g}$, where $D_{g}$ is a divisor whose support does not contain $E$.

Hence $\operatorname{div}\left(\psi^{-1}\left(g^{z}\right)\right)=-z \cdot \varepsilon \cdot E+z \cdot D_{g}$ on $X$ and $g^{z} \in F^{z^{\cdot \varepsilon}}-F^{z \cdot \varepsilon+1}$ for any integer $z \geq 0$. By (7.21.1), $G_{z \cdot \varepsilon} \rightarrow R_{z \cdot \varepsilon}$ is surjective and $U_{z \cdot \varepsilon}=0$ for $0 \leq z \cdot \varepsilon \leq \gamma+(v-1) \cdot \varepsilon$. By $(7.20 .2), \quad R^{1} \psi_{*}\left(O_{X}(-a \cdot E)\right) \cong R^{1} \psi_{*}\left(O_{X}(-(a-i) \cdot E)\right)$ for $0 \leq i \leq \gamma-1$. Therefore $U_{a-i-1}=0$ for $0 \leq i \leq \gamma-1$ by Theorem (7.2) and (7.12).

This completes the proof of Theorem (7.9).

\section{§8. The Case with $p_{a}=2$}

(8.1) Let $(W, w)$ be a normal two-dimensional singularity, $\psi:(\tilde{X}, A) \rightarrow(W, w)$ a resolution of $(W, w)$ and $\Gamma$ the dual graph of $A$. For the definition of the arithmetic genus $p_{a}(W, w)=p_{a}(\Gamma)$, we refer to $(0.7)$. For a star-shaped graph $\Gamma$, we have the following formula (Theorem (3.8) of [42]):

$$
p_{a}(\Gamma)=\max _{r \geq 1}\left\{r(g-1)+1-\sum_{k=0}^{r-1} \operatorname{deg}([k D])\right\},
$$

where $[k D]$ denotes the maximum among the set $\{$ the integral divisor $G$ on $E \mid G \leq k \cdot D$ \} for $k \in \mathbb{Z}$.

By (7.10), if $p_{a}(\Gamma)=1$, then the answer to Problem (7.6) is affirmative. So the purpose of this section is to examine the case $p_{a}(\Gamma)=2$. For the case $p_{a}(\Gamma)=2$ with $g \geq 1$, the answer is affirmative, too (Corollary (8.3) and Theorem (8.4)). Further we shall classify unsolved cases with $p_{a}(\Gamma)=2$ in Lemma (8.11) (cf. Example (8.14)).

We begin with the following:

Lemma (8.2). Let the situation be as in (6.2). If $p_{a}(\Gamma)=p_{a}(E)(=g) \geq 2$, then $a(R) \leq 2$.

Proof. We define the function $h(r)$ by

$$
h(r)=r(g-1)+1-\sum_{k=0}^{r-1} \operatorname{deg}([k D]) \quad \text { for } \quad r \geq 1 .
$$

Since $h(1)=g, p_{a}(\Gamma)=g$ is equivalent to $h(r) \leq g$ for $r \geq 0$. In particular $h(2) \leq g$ implies $\operatorname{deg}([k D]) \geq g-1$. Hence $\operatorname{deg}([k D]) \geq k \operatorname{deg}([D]) \geq k(g-1)$ for $k \geq 0$. Therefore $H^{1}\left(E, O_{E}\left(k D_{j}^{\prime}\right)=0\right.$ for $k \geq 3$.

Q.E.D.

By Corollary (7.7), we obtain the following:

Corollary (8.3). Let the situation be as in (6.2). If $(A, m)$ is Gorenstein with $p_{a}(\Gamma)=g \geq 2$, then $U=0$.

Next we shall show the following:

Theorem (8.4). Let the situation be as in (6.2). Suppose that the characteristic of the base field is not two and that the graph $\Gamma$ satisfies $p_{a}(\Gamma)=2$ with $g=1$. If $(A, m)$ is Gorenstein, then $U=0$. 
For the proof, we need the following:

Lemma (8.5). Let the situation be as in (6.2).

(1) If $p_{a}(\Gamma)=2$ and $g=1$, then there is an integer $\mu$ with $2 \leq \mu \leq 6$ such that $\operatorname{deg}([D])=-1, \operatorname{deg}([k D])=0$ for $1<k<\mu, \operatorname{deg}([\mu D]) \geq 1, \operatorname{deg}([(\mu+1) D]) \geq 0$ and $\operatorname{deg}([k D]) \geq 1$ for $k \geq \mu+2$.

(2) If $p_{a}(\Gamma)=2, g=1$ and $R(E, D)$ is Gorenstein, then $\mu \neq 5$ in (1).

(3) If $g=1$, there is no graph $\Gamma$ with the conditions: $\operatorname{deg}([D])=-1,[2 D]$ and $[3 D]$ are linearly equivalent to zero, and $\operatorname{deg}([4 D])=0$.

Proof of Lemma (8.5). (1) By Corollary (3.9) of $[42], \operatorname{deg}([D]) \leq-1$. Then $h(2)=1-\operatorname{deg}([D]) \leq 2$ by (8.1.1). Hence $\operatorname{deg}([D])=-1$. Put the integer $\mu$ by: $\mu=\min \{\alpha \in \mathbb{Z} \mid \alpha \geq 2, \operatorname{deg}([\alpha D]) \geq 1\}$. Then $h(\mu)=2-\sum_{1<k<\mu} \operatorname{deg}([k D]) \leq p_{a}(\Gamma)=2$. Therefore $\operatorname{deg}([k D])=0$ for $k$ with $1<k<\mu$ by definition of $\mu$.

To show the relation $\mu \leq 6$, we represent $D$ by:

$$
D=Q-\sum_{i=1}^{\beta} q_{i} P_{i}, \quad \text { where } \quad P_{i}=E \cap A_{i 1} \quad \text { and } \quad 1>q_{1} \geq q_{2} \geq \cdots \geq q_{\beta} \quad \text { (6.2) . }
$$

Here $\operatorname{deg}(Q)-\beta=\operatorname{deg}([D])=-1$.

Now assume $\mu \geq 7$. Then $\operatorname{deg}([2 D])=\operatorname{deg}([3 D])=\operatorname{deg}([6 D])=0$. From the relations $[(m+n) D] \geq[n D]+[m D]$ for $m, n \in \mathbb{Z}$, the relations $[6 D]=2 \cdot[3 D]=3$. $[2 D]$ follow. If we denote $[6 D]$ as $[6 D]=6 \cdot Q-\sum_{i=1}^{\beta} z_{i} \cdot P_{i}$ by integers $z_{i}, 1 \leq i \leq \beta$, then all $z_{i}$ are divisible by 6 . Hence $\operatorname{deg}([6 D]) \leq 6(\operatorname{deg}(Q)-\beta) \leq-6$. This is a contradiction.

The remaining assertions of (1) are easy to show.

(2) By the Gorenstein property of $R(E, D),[a D]=[k D]+[(a-k) D]$ for $k \in \mathbb{Z}$ and $[a D]$ is linearly equivalent to zero (see [45]). Hence we obtain $a=\mu+1$ and $\operatorname{deg}([\mu D])=1$ by (1). If $\mu=5$, then $\operatorname{deg}([2 D])=\operatorname{deg}([3 D])=\operatorname{deg}([6 D])=0$ follow. These produce a contradiction by the arguments of (1).

(3) Suppose there is a graph $\Gamma$ with conditions: $g=1, \operatorname{deg}([D])=-1,[2 D]$ and $[3 D]$ are linearly equivalent to zero, and $\operatorname{deg}([4 D])=0$. Let us denote $D$ as in (8.5.1). Then $[D]=Q-\sum_{i=1}^{\beta} P_{i}$ with $\operatorname{deg}(Q)-\beta=-1$. By the condition $\operatorname{deg}([2 D])=0$, we can easily show the following relation: $[2 D]=2 \cdot Q-\sum_{i=1}^{\beta-2} 2 \cdot P_{i}-P_{\beta-1}-P_{\beta}$. Further $\operatorname{deg}([4 D])=2 \operatorname{deg}([2 D])$ implies $[4 D]=2 \cdot[2 D]$. In particular $4 q_{i}>3$ for $i \leq \beta-2$, and $[3 D]$ is written as $[3 D]=3 \cdot Q-\sum_{i=1}^{\beta-2} 3 \cdot P_{i}-x_{\beta-1} \cdot P_{\beta-1}-x_{\beta} \cdot P_{\beta}$ by integers $x_{\beta-1}$ and $x_{\beta}$. Since $1 \leq x_{\beta} \leq x_{\beta-1} \leq 2$ (by the forms of $[2 D]$ and $[4 D]$ ) and $\operatorname{deg}([3 D])=0$, we obtain the relations $x_{\beta-1}=2$ and $x_{\beta}=1$. Hence $3 \cdot[2 D]-2 \cdot[3 D]=P_{\beta-1}-P_{\beta}$ is linearly equivalent to zero by assumption. Since $P_{\beta-1}$ and $P_{\beta}$ are distinct points on the elliptic curve, this is a contradiction.

(8.6) Proof of Theorem (8.4). As we have seen in the proof of Lemma (8.5), $a(R)=\mu+1$ and $\mu=2,3,4$, or 6 . Here $R_{1}=H^{0}\left(E, O_{E}([D])\right)=0$ implies $U_{1}=0$. 
Hence $\quad R^{1} \psi_{*}\left(O_{X}(-2 \cdot E)\right) \rightarrow R^{1} \psi_{*}\left(O_{X}\right)$ is injective. By Lemma (7.11), $R^{1} \psi_{*}\left(O_{X}(-(a(R)-1) \cdot E)\right) \rightarrow R^{1} \psi_{*}\left(O_{X}\right)$ is also injective. Hence we obtain $U_{a(R)-2}=0$. Further $U_{0}=0$ and $U_{a(R)-1}=0$ by (6.5) and Lemma (7.11).

Therefore $U=0$ for the case $a(R) \leq 4$.

For the case $a(R)=5$, we obtain the following relations: $\operatorname{dim} U=\operatorname{dim} U_{2} \leq$ $\operatorname{dim} R_{2}=\operatorname{dim} H^{0}\left(E, O_{E}([2 D])\right) \leq 1$. Then $U=0$ by Theorem (7.8).

In the case $a(R)=7$, we have the followings: $\operatorname{deg}([D])=-1, \operatorname{deg}([2 D])=$ $\operatorname{deg}([3 D])=\operatorname{deg}([4 D])=\operatorname{deg}([5 D])=0, \operatorname{deg}([6 D])=1, \operatorname{deg}([7 D])=0,[7 D]=[k D]+$ $[(7-k) D]$ for $k \in \mathbf{Z}$, and $[7 D]$ is linearly equivalent to zero. Then we can easily show that $[2 D]$ is not linearly equivalent to zero by (3) of Lemma (8.5). Hence $R_{2}=0$ and $U_{2}=0$ follow. Then $R^{1} \psi_{*}\left(O_{X}(-3 E)\right) \rightarrow R^{1} \psi_{*}\left(O_{X}\right)$ is injective. By Lemma (7.11), $R^{1} \psi_{*}\left(O_{X}(-5 E)\right) \rightarrow R^{1} \psi_{*}\left(O_{X}\right)$ is also injective. Hence $U_{4}=0$. Thus $\operatorname{dim} U=\operatorname{dim} U_{3} \leq$ $\operatorname{dim} R_{3}=\operatorname{dim} H^{0}\left(E, O_{E}([3 D])\right) \leq 1$. Therefore $U=0$ by Theorem (7.8).

This completes the proof of Theorem (8.4).

(8.7) In the rest of this paper, we discuss the case $g=0$ with $p_{a}(\Gamma)=2$. Then we obtain the equality $\operatorname{dim}\left(R^{1} v_{*}\left(O_{C}\right) / m_{R} R^{1} v_{*}\left(O_{C}\right)\right)=2$ by [42] Theorem (3.4) and Corollary (3.6). Let $\varepsilon=\varepsilon(R(E, D))$ be the integer as in (6.7). Then, by the equality $R^{1} v_{*}\left(O_{C}\right) / m_{R} R^{1} v_{*}\left(O_{C}\right)=\sum_{h=0}^{\varepsilon-1} H^{1}\left(E, O_{E}([h D])\right)$ (7.20), one of the following two cases occurs.

Case (8.7.1). There is an integer $\gamma$ with $1 \leq \gamma \leq \varepsilon-1$ such that $\operatorname{deg}([\gamma D])=-3$ and $\operatorname{deg}([i D])=-1$ for any $i \in\{i, \ldots, \varepsilon-1\}-\{\gamma\}$.

Case (8.7.2). There are two integers $\gamma_{1}$ and $\gamma_{2}$ in $\{1, \ldots, \varepsilon-1\}$ with $\gamma_{1}<\gamma_{2}$ such that $\operatorname{deg}\left(\left[\gamma_{1} D\right]\right)=\operatorname{deg}\left(\left[\gamma_{2} D\right]\right)=-2$ and $\operatorname{deg}([i D])=-1$ for any $i \in\{1, \ldots, \varepsilon-1\}-$ $\left\{\gamma_{1}, \gamma_{2}\right\}$.

Proposition (8.8). Let the situation be as in (6.2), $p_{a}(\Gamma)=2$, and $R$ satisfies the conditions in the case (8.7.1). Then

$$
\operatorname{dim}\left(m_{R} R^{1} v_{*}\left(O_{C}\right) /\left(m_{R}\right)^{2} R^{1} v_{*}\left(O_{C}\right)\right) \leq 1 .
$$

Proof. We have the relation $-3=\operatorname{deg}([\gamma D]) \geq \operatorname{deg}([\gamma-1) D])+\operatorname{deg}([D])$. Hence $\gamma=1$ in Case (8.7.1). For the function $h(r)(8.2 .1)$, we have

\begin{tabular}{ccccccc}
\hline$r$ & 1 & 2 & 3 & $\cdots$ & $\varepsilon$ & $\varepsilon+1$ \\
\hline$h(r)$ & 0 & 2 & 2 & $\cdots$ & 2 & $1-\operatorname{deg}([\varepsilon D])$ \\
\hline
\end{tabular}

In the case $\operatorname{deg}([\varepsilon D]) \geq 1$, we obtain the following relations: $\operatorname{deg}([(\varepsilon+1) D]) \geq$ $\operatorname{deg}([\varepsilon D])+\operatorname{deg}([D]) \geq-2, \operatorname{deg}([(\varepsilon+i) D]) \geq \operatorname{deg}([\varepsilon D])+\operatorname{deg}([i D]) \geq 0$ for $1<i<\varepsilon$. Then $\operatorname{dim}\left(m_{R} R^{1} v_{*}\left(O_{C}\right) /\left(m_{R}\right)^{2} R^{1} v_{*}\left(O_{C}\right)\right)=\sum_{h=\varepsilon+1}^{2 \varepsilon} \operatorname{dim}\left(H^{1}\left(E, O_{E}([h D])\right)\right) \leq 1$. This agrees with our assertion.

Let us assume $\operatorname{deg}([\varepsilon D])=0 . \quad$ Then $h(\varepsilon+1)=1$ and $h(\varepsilon+2)=-\operatorname{deg}([(\varepsilon+1) D])$. 
By the formula $(8.1 .1), \operatorname{deg}([(\varepsilon+1) D]) \geq-2$. Since $\operatorname{deg}([(\varepsilon+i) D]) \geq \operatorname{deg}([\varepsilon D])+$ $\operatorname{deg}([i D]) \geq-1 \quad$ for $\quad i \quad$ with $\quad 1<i \leq \varepsilon, \quad \operatorname{dim}\left(m_{R} \cdot R^{1} v_{*}\left(O_{C}\right) /\left(m_{R}\right)^{2} \cdot R^{1} v_{*}\left(O_{C}\right)\right)=$ $\sum_{h=\varepsilon+1}^{2 \varepsilon} \operatorname{dim}\left(H^{1}\left(E, O_{E}([h D])\right)\right) \geq 1$.

Q.E.D.

By Theorem (7.9), we obtain the following:

Corollary (8.9). Let the situation be as in (8.8). Suppose the characteristic of the base field $k$ is zero. If the singularity $(W, w)$ is Gorenstein, then $U=0$.

(8.10) In the case (8.7.2), we can not apply Theorem (7.9) directly in general.

Since $\operatorname{deg}([(m+n) D]) \geq \operatorname{deg}([m D])+\operatorname{deg}([n D])$ for $m, n \in \mathbb{Z}$, we obtain the following:

$$
\begin{array}{ll}
\operatorname{deg}([i D]) \geq-2 & \text { if } i \equiv \gamma_{1} \quad \text { or } \quad \gamma_{2}(\bmod \varepsilon), \quad \text { and } i \geq 0, \\
\operatorname{deg}([i D]) \geq 0 & \text { if } i \equiv 0(\bmod \varepsilon), \quad \text { and } i \geq 0, \\
\operatorname{deg}([i D]) \geq-1 & \text { if } i \equiv 0, \gamma_{i}, \quad \text { or } \quad \gamma_{2}(\bmod \varepsilon), \text { and } i \geq 0 .
\end{array}
$$

In particular $a(R) \equiv \gamma_{1}$ or $\gamma_{2}(\bmod \varepsilon)$.

Lemma (8.11). Let the situation be as in (6.2), $p_{a}(\Gamma)=2$, and $\mathbb{R}$ satisfies the conditions in the case (8.7.2). Suppose that $\mathbb{R}(E, D)$ is Gorenstein and $\operatorname{dim}\left(m_{R} \cdot R^{1} v_{*}\left(O_{C}\right) /\left(m_{R}\right)^{2} \cdot R^{1} v_{*}\left(O_{C}\right)\right)=2$. Then:

(1) In the case $a(R)=\gamma_{1}+u \cdot \varepsilon$ for an integer $u$, we have $u \geq 3$ and

$$
\begin{array}{ll}
\operatorname{deg}([i D])=-2 & \text { if } i=\gamma_{1}+k \cdot \varepsilon \text { for } 0 \leq k \leq u \\
\operatorname{deg}([i D])=0 & \text { or } i=\gamma_{2}+k \cdot \varepsilon \text { for } 0 \leq k \leq u-2, \\
\operatorname{deg}([i D])=-1 \quad \text { for other indices } i, \quad 0 \leq i \leq a(R) .
\end{array}
$$

In particular $p_{g}(R)=2 \cdot u$.

(2) In the case $a(R)=\gamma_{2}+u \cdot \varepsilon$ for an integer $u$, we have $u \geq 2$, and

$$
\begin{array}{ll}
\operatorname{deg}([i D])=-2 & \text { if } i=\gamma_{1}+k \cdot \varepsilon \text { for } 0 \leq k \leq u-1 \\
\operatorname{deg}([i D])=0 & \text { or } i=\gamma_{2}+k \cdot \varepsilon \text { for } 0 \leq k \leq u, \\
\operatorname{deg}([i D])=-1 \quad \text { for other indices } i, \quad 0 \leq i \leq a(R) .
\end{array}
$$

In particular $p_{g}(R)=2 \cdot u+1$. 
By the above data, we obtain: $\varepsilon \geq 4, \gamma_{1} \geq 1, \gamma_{2} \geq 3$ and $a(R) \geq 13$ for (1) of Lemma (8.11), $a(R) \geq 11$ for (2) of Lemma (8.11). Hence we obtain the following by Theorem (7.9).

Corollary (8.12). Let the situation be as in (6.2). Suppose the characteristic of the base field $k$ is zero and the graph $\Gamma$ satisfy $p_{a}(\Gamma)=2$ with $a(R) \leq 10$. If the singularity $(W, w)$ is Gorenstein, then $U=0$.

(8.13) Proof of Lemma (8.11). We shall show the assertion for the case (1). First $-2=\operatorname{deg}([a D])=\operatorname{deg}\left(\left[\left(\gamma_{1}+k \cdot \varepsilon\right) D\right]\right)+\operatorname{deg}([((u-k) \cdot \varepsilon) D]) \geq \operatorname{deg}\left(\left[\gamma_{1} D\right]\right)+$ $u \operatorname{deg}([\varepsilon D]) \geq-2$, implies $\operatorname{deg}\left(\left[\left(\gamma_{1}+k \cdot \varepsilon\right) D\right]\right)=-2$, and $\operatorname{deg}([k \in D])=0$ for $0 \leq k \leq$ $u$. For the function $h(r)(8.2 .1)$, we have:

\begin{tabular}{ccccccccccc}
\hline$r$ & 1 & $\cdots$ & $\gamma_{1}$ & $\gamma_{1}+1$ & $\cdots$ & $\gamma_{2}$ & $\gamma_{2}+1$ & $\cdots$ & $\varepsilon$ & $\varepsilon+1$ \\
\hline$h(r)$ & 0 & $\cdots$ & 0 & 1 & $\cdots$ & 1 & 2 & $\cdots$ & 2 & 1 \\
\hline
\end{tabular}

Assertion (8.13.1). There is an integer $\delta$ with $\varepsilon<\delta<2 \cdot \varepsilon$ and $R_{\delta} \neq 0$.

Proof of Assertion (8.13.1). Suppose $R_{k}=0$ for $k$ with $\varepsilon<k<2 \cdot \varepsilon$. Then $\operatorname{deg}([k D]) \leq-1$ for $k$ with $\varepsilon<k<2 \cdot \varepsilon$. We can easily show $h(2 \varepsilon) \geq 3$ by using the relations $\operatorname{deg}\left(\left[\left(\gamma_{1}+\varepsilon\right) D\right]\right)=\operatorname{deg}\left(\left[\left(\gamma_{2}+\varepsilon\right) D\right]\right)=-2$. This contradicts the assumption $p_{a}(\Gamma)=2$.

Q.E.D. for (8.13.1).

By the Serre duality: $R_{k} \leftrightarrow H^{1}\left(E, O_{E}([(a-k) D])\right)($ see $[45]), \operatorname{deg}([(a-\delta) D]) \leq$ -2. Hence $\delta=2 \varepsilon+\gamma_{1}-\gamma_{2}, \quad \operatorname{deg}([(a-\delta) D])=\operatorname{deg}\left(\left[\left(\gamma_{2}+(u-2) \cdot \varepsilon\right) D\right]\right)=-2$ and $\operatorname{deg}\left(\left[\left(2 \varepsilon+\gamma_{1}-\gamma_{2}\right) D\right]\right)=0$. In particular $\varepsilon+\gamma_{2}=2 \cdot \varepsilon+\gamma_{1}-\gamma_{2}$.

The remaining assertions are not difficult to show.

The arguments in the proof of (2) are similar to (1). So we shall omit them.

Example (8.14). Let the situation be as in Theorem (6.3) and the dual graph $\Gamma$ of $f^{-1}(w)$ be as follows:

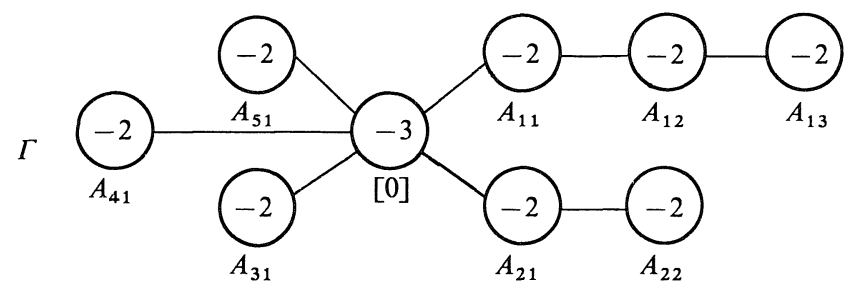

Then Pinkham's construction $R(E, D)$ is a Gorenstein domain with $p_{a}(\Gamma)=2$ and has the invariants $\gamma_{1}=1, \gamma_{2}=3, \varepsilon=4$, and $a(R)=11$ in the terminologies of (2) of Lemma (8.12). In fact, with $D=3 P_{0}-\frac{3}{4} P_{1}-\frac{2}{3} P_{2}-\frac{1}{2} P_{3}-\frac{1}{2} P_{4}-\frac{1}{2} P_{5}$, where $P_{i}=E \cap$ $A_{i 1}, 1 \leq i \leq 5, P_{0}$ a point of $E$, and $D^{\prime}=\frac{3}{4} P_{1}+\frac{2}{3} P_{2}+\frac{1}{2} P_{3}+\frac{1}{2} P_{4}+\frac{1}{2} P_{5}, K_{E}+D^{\prime}-11 D$ is linearly equivalent to zero on $E$. Further we have 


\begin{tabular}{crrrrrrrrrrr}
\hline$r$ & 1 & 2 & 3 & 4 & 5 & 6 & 7 & 8 & 9 & 10 & 11 \\
\hline $\operatorname{deg}\left(D^{(r)}\right)$ & -2 & -1 & -2 & 0 & -2 & 0 & -2 & 0 & -1 & 0 & -2 \\
\hline$h(r)$ & 0 & 1 & 1 & 2 & 1 & 2 & 1 & 2 & 1 & 1 & 0 \\
\hline
\end{tabular}

$$
\operatorname{deg}([r D]) \geq-1 \text { and } h(r) \leq 1 \text { for } r \geq 12 .
$$

(In general we have the relation $p_{a}(\Gamma)=\max _{1 \leq r \leq a(R)+1} h(r)$ ).

Hence $p_{g}(R(E, D))=5$.

We shall study our $U$. Firstly $U_{k}=0$ for $k \geq a=11$, by the relation $U_{k}=$ $\operatorname{Ker}\left\{R^{1} \psi_{*}\left(O_{X}(-(k+1) E)\right) \rightarrow R^{1} \psi_{*}\left(O_{X}(-k E)\right\}\right.$ (see $\left.\S \S 1,5,6\right)$. Secondly $U_{0}=0$ by the fact (6.5). Thirdly $U_{k}=0$ for $k=1,2,3,5,7,9$. Hence $R^{1} \psi_{*}\left(O_{X}(-k E)\right) \rightarrow R^{1} \psi_{*}\left(O_{X}\right)$ is injective for $k \leq 4$. By Lemma (7.16), we obtain

$$
\operatorname{dim} U=\sum_{k \geq 5} \operatorname{dim}\left(\xi_{k}\left(\operatorname{Ker}\left\{R^{1} \psi_{*}\left(O_{X}(-k E)\right) \rightarrow R^{1} \psi_{*}\left(O_{X}\right)\right\}\right)\right) \leq \sum_{k \geq 5} \operatorname{dim} H^{1}\left(E, O_{E}(k D)\right)=3 .
$$

From here we assume $(W, w)$ is a Gorenstein singularity.

We have the injections $R^{1} \psi_{*}\left(O_{X}(-(12-k) E)\right) \rightarrow R^{1} \psi_{*}\left(O_{X}\right)$ for $k \leq 4$ by Lemma (7.11). Hence $U_{8}=U_{10}=0$. Further if we assume that the characteristics of the base field is not two, $\operatorname{dim} U \neq 1$ by Theorem (7.8). Hence $\operatorname{dim} U=0$, or 2 .

We do not know an example with $\operatorname{dim} U=2$ for this graph.

\section{References}

[1] Artin, M., Some numerical criteria for contractability of curves on algebraic surfaces, Amer. J. Math., 84 (1962), 485-496.

[ 2 ] - On isolated rational singularities of surfaces, Amer. J. Math., 88 (1966), 129-136.

[3] Bourbaki, N., Éléments de Mathématique, Algèbre Commutative, Hermann, Paris, 1961, 1964, 1965.

[4] Boutot, J.-F., Singularités rationelles et quotient par les groupes reductifs, Invent. Math., 88 (1987), $65-68$.

[ 5] Bruns, W., The canonical module of an associated graded ring, Arch. Math., 47 (1986), 320-323.

[6] Cavaliere, M. P., Niesi, G., On Serre's conditions in the form ring of an ideal, J. Math. Kyoto Univ., 21-3 (1981), 537-546.

[7] Demazure, M., Anneaux gradués normaux. Preprint. Ecole Polytechnique (1979).

[ 8 ] Elkik, R., Singularités rationelles et déformations, Invent. Math., 47 (1978), 139-147.

[9] Flenner, H., Rationale quasi-homogene Singularitäten, Arch. Math., 36 (1981), 35-44.

[10] Giraud, J., Improvement of Grauert-Riemenschneider's Theorem for a normal surface, Ann. Inst. Fourier Grenoble., 32-4 (1982), 13-23.

[11] Goto, S., Shimoda, Y., On the Rees algebra of Cohen-Macaulay local rings, in "Commutative Algebra; analytic method", Lecture Notes in Pure and applied Math. No. 68 (1982), 201-231, Marcel Dekker.

[12] Goto, S., Watanabe, K.-i., On graded rings I., J. Math. Soc. Japan, 30 (1978), 179-213.

[13] Grauert, H., Riemenschneider, O., Verschwindungssätze fur analytische Kohomologiegrouppen auf komplexen Raumen, Invent. Math., $\mathbb{1 1}$ (1970), 263-292.

[14] Grothendieck, A., Local cohomology, Lecture Note in Math., 41, Springer, 1966.

[15] Grothendieck, A., Dieudonne, J., Eléments de Géométri Algébrique II.III.IV-2, Publ. IHES. 4 (1961), 8 (1961), 11 (1961), 17 (1963), 24 (1965).

[16] Hartshorne, R., Residues and Duality, Lecture Note in Math., 20, Springer, 1966. 
[17] Herzog, J., Vasconcelos, W. V., On the divisor class group of Rees algebras, J. of Algebra, 93 (1985), 182-188.

[18] Herzog, J., Simis, A., Vasconcelos, W. V., On the canonical module of the Rees algebra and the associated graded ring of an ideal, J. of Algebra, 105 (1987), 285-302.

[19] Hidaka, F., Normal surface singularities associated to ruled surfaces, (in Japanese), Proceeding of Symposium on "COMMUTATIVE RINGS", 7 (1985), 145-159.

[20] - A projective contractibility criteria and its applications, in preparation.

[21] Hidaka, F., Tomari, M., On singularities arising from the contraction of the minimal section of ruled surfaces, Manuscripta math., 65 (1989), 329-347.

[22] Hochster, M., Ratliff, L. J., Jr., Five theorems on Macaulay rings, Pacific J. Math., 44-1 (1973), $147-172$.

[23] Hochster, M., Roberts, J., Rings of invariants of reductive groups acting on regular rings are CohenMacaulay, Adv. in Math., 13 (1974), 115-175.

[24] Kempf, G., Knudesen, F., Mumford, D., Saint-Donat, B., Toroidal embeddings I, Lecture Note in Math., 339, Springer, 1973.

[25] Laufer, H. B., Normal two-dimensional singularities, Ann. Math. Studies, 71, Princeton Univ. Press, Princeton, 1971.

[26] —- On minimally elliptic singularities, Amer. J. Math., 99 (1977), 1257-1295.

[27] $\longrightarrow$, Tangent cones for deformations of two-dimensional quasi-homogeneous singularities, Preprint 1986.

[28] Lipman, J., Rational singularities, with applications to algebraic surfaces and unique factorization, Publ. I.H.E.S., 36 (1969), 195-279.

[29] — Desingularization of two-dimensional schemes, Ann. of Math., 107 (1978), 151-207.

[30] - Dualizing sheaves, differentials and residues on algebraic varieties, Asterisque, 117, Societe Math. de France, 1984.

[31] Morales, M., Resolution of quasi-homogeneous singularities and plurigeneras, Composito Math., 64 (1987), 311-327.

[32] Orlik, P., Wagreich, Ph., Isolated singularities of algebraic surface with $\mathrm{C}^{*}$-action, Ann. of Math., 93 (1970), 205-228.

[33] —, Algebraic surfaces with $k^{*}$-action, Acta Mathematica, 138 (1977), 43-81.

[34] Pinkham, H., Normal surface singularities with C*-action, Math. Ann., 227 (1977), 183-193.

[35] Reid, M., Canonical 3-folds, Journees de geometrie algebrique d'Angers 1979, "Algebraic geometry" edited by A. Beauville, Sijthhoff and Noordhoff, 1980, 273-310.

[36] Rees, D., Notes of lectures given at Nagoya university, i982-1983.

[37] Riemenschneider, O., Bemerkungen zur Deformationstheorie nichtrationaler Singularitäten, Manuscripta math., 14 (1974), 91-99.

[38] Sakai, F., Weil divisors on normal surfaces, Duke Math. J., 51 (1984), 877-887.

[39] Samuel, P., Lectures on Unique Factorization Domains, Tata Inst. Fund. Res., Bombay, 1964.

[40] Shimoda, Y., On Rees algebras of ideals generated by a subsystem of parameters, J. Math. Kyoto Univ., 21-2 (1981), 231-238.

[41] Tomari, M., A $p_{g}$-formula and elliptic singularities, Publ. Res. Inst. Math. Scien., Kyoto Univ., 21 (1985). 297-354.

[42] - Maximal-ideal-adic filtration on $R^{1} \psi_{*} O_{\tilde{v}}$ for normal two-dimensional singularities, Advanced Studies in Pure Mathematics, 8 (1986), "Complex Analytic Singularities, Tsukuba-Kyoto, 1984", 633-647, KINOKUNIYA-NORTH-HOLLAND.

[43] Wagreich, Ph., Elliptic singularities of surfaces, Amer. J. Math., 92 (1970), 419-454.

[44] Watanabe, K.-i., Certain invariant subrings are Gorenstein I.II, Osaka J. Math., 11 (1974), 1-8, $379-388$.

[45] - Some remarks concerning Demazure's construction of normal graded rings, Nagoya Math. J., 83 (1981), 203-211.

[46] — Rational singularities with $k^{*}$-action, In Commutative algebra. Proc. Trento Conf. edited by S. Greco and G. Valla, Lecture Notes in Pure and applied Math. No. 84 (1983), 339-351, Marcel Dekker. 
[47] Watanabe, Kimio., A revised version of "On plurigenera of normal isolated singularities I, Math. Ann., 250 (1980), 65-94. “RIMS Kokyuroku, No. 415 (1981), Kyoto University.

[48] Yau, Stephen S.-T., Normal two-dimensional elliptic singularities, Trans. of A.M.S., 254 (1979), 117-134.

[49] - On maximally elliptic singularities, Trans. of A.M.S., 257 (1980), 269-329.

[50] - Hypersurface weighted dual graphs of normal singularities of surfaces, Amer. J. Math., 101 (1979), 761-812.

[51] - Sheaf cohomology on 1-convex manifolds, "Recent Developments in Several Complex Variables" Ann. of Math. Studies, Princeton Univ., 100 (1981), 429-452.

[52] Fossum, R., Foxby, H.-B., The category of graded modules, Math. Scand., 35 (1974), 288-300.

Note Added in Proof.

(1) In (3.9) we need the assumption " $G$ is generated by $G_{1}$ over $G_{0}$ ". In fact, without this assumption, let $A=\mathbb{C}\{x, y, z, w\} /\left(x^{2}+y^{3}+z^{10}+w^{15}\right)$ with the "natural" filtration so that $G=$ $\mathbb{C}[x, y, z, w] /\left(x^{2}+y^{3}+z^{10}+w^{15}\right)$. Then $a(G)=0$, but $\omega_{X}=O_{X}(1)=O_{X}(-E)$ is not generated by $H^{0}\left(X, \omega_{X}\right)=m=F^{1}=F^{2}$, because $G_{1}=0$. Accordingly, we add the same assumption to (4.9). Also, the statement "if and only if" in (ii) of (4.11) should be replaced by "if".

(2) Actually, there is a counter example for (7.6.1) in positive characteristics (see [21]). In characteristic zero, we still do not know the answer. 